$200)$
$+67 \mathrm{~N}$

mo. 129

UIITHD STATHS DIRPARTYENT OF THE INTFRIOR

GROLOGICAI SURVEY

a cataiog of STUDY Material

OF RADIOACTIVR MINERATS

by

T. W. Stern

October 1950

Trace Mlements Investigations Report 129 
CONTENTS

Page

Introduction $\ldots \ldots \ldots \ldots \ldots \ldots \ldots \ldots \ldots \ldots \ldots \ldots \ldots \ldots \ldots \ldots$

Group A. Uranium and thorium minerals ................ 5

Group B. Minerals with minor amounts of uranium and thorium .....................62

Group C. Synthetic uranium compounds prepared by the U. S. Geological Survey ............. 79 
A CATALOG OF STUDY MATERIAL OF RADIOACTIVE MINERAIS

by T. W. Stern

Introduction

This catalog is a list of specimens of radioactive minerals for which the Geological Surrey has been collecting optical, X-ray diffraction, and other data. The specimens are in the study collections of the U. S. National Museum, the Mineralogical Museum of Harvard University (including some specimens on loan at Harvard from the American Museum of Natural History), and the Geological Survey. The listing does not include all specimens in these institutions, but it does include those specimens for which $\mathrm{X}$-ray patterns can be made available as well as representative specimens from significant localities. In addition, references are given to material on deposit, at the U.S. National Museum, that has been described in the literature. Because of the rarity of much of the material here listed, requests for study material must be considered individually. Regardless of the quantity of material, any request granted by the U. S. National Museum will have to be accepted on the terms laid down by that organization to conserve limited or rare minerals.

Those minerals that show external crystal form but do not give X-ray powder diffraction patterns are listed with the word "ignited" after the $\mathrm{X}$-ray powder diffraction photograph number. The ignition was done in air to red heat except where noted. 
In the course of work at Harvard on radioactive minerals many mineral species have been designated as "phases". Many of these phases have been identified as valid species already known; some new species have been established. Many phases remain unidentified and these present some problems. Some may be mixtures and others may not be uranium minerals. Phase numbers assigned by Professor Frondel of Harvard University are given in the list for some species.

The grouping of the minerals in this list follows that in "A glossary of uranium- and thorium-bearing minerals," by Judith Weiss Frondel and Michael Fleischer (U. S. Geological Survey Circular 74). Optical properties are given for most of the minerals except the silicates, and such data are taken largely from volumes 1 and 2 of the 7th Edition of Dana's System of Mineralogy.

The following entry, partly fictitious for illustration's sake, shows the form used in the list:

\begin{tabular}{|c|c|c|c|}
\hline $\begin{array}{l}\text { Mineral and identi- } \\
\text { fying number }\end{array}$ & Location & $\begin{array}{l}\text { Ref. no. of } \\
\text { X-ray patterns }\end{array}$ & Quantity \\
\hline $\begin{array}{l}\text { Vanoxite I/ } \\
\text { USNM } 95059\end{array}$ & $\begin{array}{l}\text { Bill Bryan claim, } \\
\text { Wild Steer Canyon } \\
\text { Montrose County, }\end{array}$ & TE 474 & $\begin{array}{l}\text { USGS-XS } \\
\text { USNM-150 } \\
\text { BMM-3x } 3, M\end{array}$ \\
\hline
\end{tabular}

IJ Hess, F. L., U. S. Geol. Survey Bull. 750-D, 63 (1925). The first column gives the mineral name and the institutional number of the specimen; the second column, the locality; the third, the reference number of any $X$-ray diffraction powder patterns that have been made; and the fourth, the quantity of material available. In the example given above the Geological Survey has enough material for X-ray 
and spectrographic analysis; the U. S. National Museum has about 150 grams of this mineral; and the Harvard Mineralogical Museum has a moderately incrusted specimen measuring about 3 by 3 centimeters. The kindness of Dr. George Switzer of the U. S. National Museum and Professor Clifford Frondel of Harvard University for their many helpful suggestions is gratefully acknowledged. Joseph Berman, Theodore Botinelly, J. H. Eric, Judith Weiss Frondel, K. J. Murata, John C. Rabbitt, and Jane Titcomb of the Geological Survey also have aided in the work. 
The following abbreviations are used:
AMNH
American Museum of Natural History.
C
Enough material for one chemical analysis.
H
Heavily incrusted specimen with area of specimen given in centimeters.
Har. Harvard X-ray powder pattern.
HMM Harvard Mineralogical Museum.
Inv. Investigations Section, Geochemistry and Petrology Branch, U. S. Geological Survey.
L
Lightly incrusted specimen with area given in centimeters.
M
Moderately incrusted specimen with area given in centimeters.
S
Enough material for one spectrographic analysis.
TE
Trace Elements Section, Geochemistry and Petrology Branch, U. S. Geological Survey.
USGS U. S. Geological Survey.
USNM U. S. National Museum.
USNM C U. S. National Museum Canfield Collection.
USNM $R \quad U$. S. National Museum Roebling Collection.
$\mathrm{X}$
Enough material for one X-ray powder pattern. 
GROUP A. URANIUM AND THORIUM MINERAIS

AMPANGaBeITE $(\mathrm{Y}, \mathrm{Er}, \mathrm{U}, \mathrm{Ca}, \mathrm{Th})_{2}(\mathrm{Cb}, \mathrm{Ta}, \mathrm{Fe}, \mathrm{T} 1)_{7} \mathrm{O}_{18}$ ?

Optics I/

In transmitted light, red brown in color and isotropic (metamict). $n=2.13 \pm 0.03$ (Ampangabé).

Specimen Data

Mineral and identi- Location

fying number

Ampangabeite

USNM C4020

Samiresy, Betafo,

Madagascar

Ref. no. of

X-ray patterns

Quantity

USGS-XS

USNM-100 g

Ampangabe1te

Ambatofosikely,

HMM 88874

Madagas car

Har. 11299

Not ignited,

no pattern

Ampangabeite

Ambatofosikely,

Madagascar

Har. 11302

Ignited,

faint pattern

I) Larsen, E. S., U. S. Geol. Survey Bull. 679, 39 (1921).

ANDERSONITE $\mathrm{Na}_{2} \mathrm{Ca}\left(\mathrm{UO}_{2}\right)\left(\mathrm{CO}_{3}\right)_{3} \cdot 6 \mathrm{H}_{2} \mathrm{O}$

Optics I/

$\begin{array}{llll} & \mathrm{n} & \text { Dichroism } & \\ 0 & 1.520 & \text { Colorless } & \\ \mathrm{E} & 1.540 & \text { Pale yellow } & \text { Uniaxial positive }\end{array}$

Specimen Data

Mineral and Identi-
fying number

Andersonite I/

USNM
Location

Hillside mine,

Yavapai County,

Ariz.
Ref. no. of

X-ray patterns

Inv. 2791

USNM-2 mg

If Milton, Charles, U. S. Geol. Survey, personal communication. 
AUTUNITE Ca $\left(\mathrm{UO}_{2}\right)_{2}\left(\mathrm{PO}_{4}\right)_{2} \cdot 10-12 \mathrm{H}_{2} \mathrm{O}$

Optics

Orientation $n$ (Autun) 1/ $n$ (Maryland)2/
(E) $\mathrm{X}$
1.553
1.555
(o) $\frac{Y}{Z}$
1.575
1.575
1.578

n $3 /$

Dichroism

1.58-1.59 Colorless to pale yellow

Yellow to rark yellow

1.59-1.60 Yellow to dark yellow

Some anomalous biaxial negative autunite is uniaxial. The biaxiality is dependent on the water content of the crystals and $2 \mathrm{~V}$ decreases with decreasing water content. In the biaxial material, $2 \mathrm{~V}$ ranges from $0^{\circ}$ to about $53^{\circ}$ and usually is $10^{\circ}$ to $30^{\circ}$ with $\mathrm{r}>\mathrm{V}$, strong; $\mathrm{Z}=\mathrm{c}$ and $\mathrm{Y}$ is parallel to one of the diagonals of the square tablets. The tetragonal dehydration product, meta-autunite I, also shows a sectoral, biaxial character with $2 \mathrm{~V}$ decreasing to 00 with decreasing water content; $Z=c$ and $Y$ is perpendicular to the edges of the tablets. The indices of refraction in both hydrates increase with decreasing water content. Some of the reported descriptions of autunites probably refer to meta-autunite I

Specimen Data

\begin{tabular}{|c|c|c|c|}
\hline $\begin{array}{l}\text { Mineral and identi- } \\
\text { fying number }\end{array}$ & Iocation & $\begin{array}{l}\text { Ref. no. of } \\
\text { X-ray patterns }\end{array}$ & Quantity \\
\hline $\begin{array}{l}\text { Autunite } \\
\text { HMM } \\
\text { Phase } 14\end{array}$ & $\begin{array}{l}\text { Gross Green, } \\
\text { Ruggles mine, } \\
\text { N. H. }\end{array}$ & Har. 1640 & -- \\
\hline $\begin{array}{l}\text { Autunite } \\
\text { MMM } \\
\text { Phase } 14\end{array}$ & Argentina & Har. 5546 & --- \\
\hline $\begin{array}{l}\text { Autunite } \\
\text { USNM } 87239\end{array}$ & $\begin{array}{l}\text { Mount Painter, } \\
\text { Victoria, Australia }\end{array}$ & --- & $\begin{array}{l}\text { USGS-XS } \\
\text { USNM- } 3 \times 3, M\end{array}$ \\
\hline $\begin{array}{l}\text { Autunite } \\
\text { USNM R5672 }\end{array}$ & $\begin{array}{l}\text { Mount Painter, } \\
\text { Victoria, Australia }\end{array}$ & -.- & $\begin{array}{l}\text { USGS-XS } \\
\text { USNM- } 3 \times 6, M\end{array}$ \\
\hline $\begin{array}{l}\text { Autunite? } \\
\text { HMM(CC-4) } \\
\text { Phase } 27\end{array}$ & $\begin{array}{l}\text { Cornwall, } \\
\text { England }\end{array}$ & Har. 3936 & $\mathrm{HMM}-6 \times 6, M$ \\
\hline $\begin{array}{l}\text { Autunite } \\
\text { USNM C } 4384\end{array}$ & $\begin{array}{l}\text { Autun, } \\
\text { France }\end{array}$ & --- & $\begin{array}{l}\text { USGS-XS } \\
\text { USNM-20 g }\end{array}$ \\
\hline $\begin{array}{l}\text { Autunite } \\
\text { USNM } 79981\end{array}$ & $\begin{array}{l}\text { Autun, } \\
\text { France }\end{array}$ & TE 3122 & USNM-20 g \\
\hline
\end{tabular}


Autunite---Specimen Data (Cont.)

Mineral and identi- Location

Ref. no. of

fying number

X-ray patterns

Autunite

USNM R5676

Lurissa,

Italy

Autunite?

TE

Mina de Urgeiriça,

Portugal

Autunite

USNM R5675

Lauter,

Saxony

Quantity

USGS-XS

USNM-12×8,M

USGS-XS

USGS-XS

USNM- $3 \times 3, M$

If Michel-Levy, A., and Lacroix, Alfred, Les minéraux des roches, 157 (1888).

2/ Shannon, E. V., Am. Mineralogist 11, 35 (1926).

3) Meixner, Heinz, Chemie der Erde 12, 433 (1940).

BASSETITE $\mathrm{Fe}\left(\mathrm{UO}_{2}\right)_{2}\left(\mathrm{PO}_{4}\right)_{2} \cdot 8 \mathrm{H}_{2} \mathrm{O}$

Optics

$\begin{array}{cc}\text { Orientation } & n(\mathrm{Na}) \\ \mathrm{X} \text { b } & (\sim 1.56) \\ \mathrm{Z} \wedge \mathrm{c}-4^{\circ} & 1.574 \\ & 1.580\end{array}$

Pleochroism

Pale yellow

Deep yellow

Deep yellow.

Biaxial negative (-) $2 \nabla \sim 62^{\circ}$

Specimen Data

\begin{tabular}{|c|c|c|c|}
\hline $\begin{array}{l}\text { Mineral and identi- } \\
\text { fying number }\end{array}$ & Location & $\begin{array}{l}\text { Ref. no. of } \\
\text { X-ray patterns }\end{array}$ & Quantity \\
\hline $\begin{array}{l}\text { Bassetite } \\
\text { USNM R7906 } \\
\text { Phase } 15\end{array}$ & $\begin{array}{l}\text { Cornwall, } \\
\text { England }\end{array}$ & $\mathrm{TE} 3126$ & USNM- $3 \times 3, L$ \\
\hline $\begin{array}{l}\text { Bassetite } \\
\text { USNM C4386 }\end{array}$ & $\begin{array}{l}\text { Cornwall, } \\
\text { England }\end{array}$ &.- & $\begin{array}{l}\text { USGS-XS } \\
\text { USNM-6x6,L }\end{array}$ \\
\hline $\begin{array}{l}\text { Bassetite } \\
\text { AMNH } 19786\end{array}$ & $\begin{array}{l}\text { Wheal Basset mine } \\
\text { Cornwall, England }\end{array}$ & -.- & $\mathrm{HMM}-3 \times 3, \mathrm{~L}$ \\
\hline
\end{tabular}




\section{BAILEYITE $\mathrm{Mg}_{2}\left(\mathrm{UO}_{2}\right)\left(\mathrm{CO}_{3}\right)_{3} \cdot 18 \mathrm{H}_{2} \mathrm{O}$}

\section{Optics I/}

$\begin{array}{cc}\text { Orientation } & \mathrm{n} \\ \mathrm{X} & 1.455 \\ \mathrm{Y} & 1.490 \\ \mathrm{Z} & 1.500\end{array}$

Pleochroism

Pinkish?

Pale yellow

Pale yellow

Biaxial negative (-) $2 \mathrm{~V}=30^{\circ}$

Specimen Data
Mineral and identi-
fying number
Location
Ref. no. of
Quantity
X-ray patterns
Bayleyite
Hillside mine,
Inv. 2777
Yavapai County, Ariz.
None,
bayleyite
Meta-bayleyite $\mathrm{Mg}_{2}\left(\mathrm{UO}_{2}\right)\left(\mathrm{CO}_{3}\right)_{3} \cdot ? \mathrm{H}_{2} \mathrm{O}$
Optics

$\begin{array}{lc} & \mathrm{n} \\ \mathrm{X} & 1.502 \\ \mathrm{Z} & 1.551 \\ & \text { Specimen } \\ \text { Data }\end{array}$

Mineral and identifying number

Meta-bayleyite

USNM ?
Location

Hillside mine

Yavapai County, Ariz.
Ref. no. of

X-ray patterns

Inv. 4004
Quantity

USNM-smalI quantities can be separated from $500 \mathrm{~g}$ of ro

If Milton, Charles, U. S. Geol. Survey, personal communication.

\section{BECQUEREIITE $2 \mathrm{UO}_{3} \cdot 3 \mathrm{H}_{2} \mathrm{O}$ ? \\ Optics}

Yellow in transmitted light. With increasing temperature $2 \mathrm{~V}$ decreases, and the mineral is uniaxial at about $1000^{\circ} \mathrm{C} .1$ 
Becquerelite---Optics (Cont.)

$\begin{array}{ccc}\text { Orientation } & \mathrm{n} / 2 / \\ \mathrm{X} & \mathrm{c} & 1.735 \\ \mathrm{Y} & \mathrm{b} & 1.820 \\ \mathrm{Z} & \mathrm{a} & 1.830\end{array}$

Pleochroism

Colorless Biaxial negative (-)

Light yellow $2 \mathrm{~V}=31^{\circ}(\mathrm{Na})$

Dark yellow

$r>v$, marked.2/

Specimen Data

\section{Mineral and identi- fying number}

Becquerelite

USMM 104784

Becquerelite

USNM R5896

Becquerelite

USMM R6701

Becquerelite

HMM 87086

Phase 78

Becquerelite

USNM R8391
Location

Chinkolobwe,

Belgian Congo

Chinkolobwe,

Belgian Congo

Chinkolobwe,

Belgian Congo

Katanga,

Belgian Congo
Ref. no of

X-ray patterns

Quantity

USGS-XS

USNM-6×6,M

USGS-XS

USNM-300 g

USGS-XS

USNM- $6 \times 6, M$

Har. 1193

HMM- $1 \mathrm{~g}$

Kalongwe,

Belgian Congo

USGS-XS

If Schoep, Alfred, Soc. géol. Belgique Bull. 33, 197 (1923).

2) Berman, Harry, in Palache, Charles, and Berman, Harry, Am. Mineralogist 18, 20 (1933). Billiet, V., Soc. frang. minéralogie Bull. 49, 136 (1926), gives nX $=1.750, n Y-1.87, \mathrm{nZ}-1.88$.

BETAFITE $(\mathrm{U}, \mathrm{Ca})(\mathrm{Cb}, \mathrm{Ta}, \mathrm{Ti})_{3} \mathrm{O} \cdot \mathrm{nH}_{2} \mathrm{O}$ ?

Optics I/

In transmitted light nearly colorless and isotropic. $n=1.915 \pm 0.02$ (Antaifasy), 1.925 \pm 0.01 (Betafo), $1.92-1.96$ (samiresite).

Specimen Data

Mineral and identi- Location

fying number

Betafite USNM 96864
Ref. no. of

X-ray patterns

Madagascar
Quantity

USGS-XS

USNM-500 g 
Betafite---Specimen Data (Cont.)

Blomstrandite--Synonym of betafite

\begin{tabular}{|c|c|c|c|}
\hline $\begin{array}{l}\text { Mineral and identi- } \\
\text { fying number }\end{array}$ & Location & $\begin{array}{l}\text { Ref. no. of } \\
\text { X-ray patterns }\end{array}$ & Quantity \\
\hline $\begin{array}{l}\text { Blomstrandite } \\
\text { USNM } 92976\end{array}$ & $\begin{array}{l}\text { Betafo, } \\
\text { Madagascar }\end{array}$ & TE 649 & $\begin{array}{l}\text { USGS-XS } \\
\text { USMM-75 g }\end{array}$ \\
\hline $\begin{array}{l}\text { Blomstrandite } \\
\text { HMM } 87873\end{array}$ & $\begin{array}{l}\text { Tongafeno, } \\
\text { Madagascar }\end{array}$ & $\begin{array}{l}\text { Har. } 2020 \\
\text { Ignited }\end{array}$ & -- \\
\hline \multicolumn{4}{|c|}{ Samiresite--Symonym of betafite } \\
\hline $\begin{array}{l}\text { Samiresite } \\
\text { USNM C3958 }\end{array}$ & $\begin{array}{l}\text { Samiresy, } \\
\text { Madagascar }\end{array}$ & $-\infty$ & $\begin{array}{l}\text { USGS-XS } \\
\text { USNM-75 g }\end{array}$ \\
\hline $\begin{array}{l}\text { Samiresite } \\
\text { HMM } 878.13\end{array}$ & $\begin{array}{l}\text { Samiresy, } \\
\text { Madagascar }\end{array}$ & $\begin{array}{l}\text { Har. } 344 \\
\text { Not ignited, } \\
\text { no pattern }\end{array}$ & --- \\
\hline
\end{tabular}

BRANNERITE (U, $\mathrm{Ca}, \mathrm{Fe}, \mathrm{Y}, \mathrm{Th})_{3} \mathrm{Ti}_{5} \mathrm{O}_{16}$ ?

Optics

In transmitted light, yellowish green. Metamict. Isotropic. Indices:1/ $n_{\mathrm{Li}} 2.26 \pm 0.02, \mathrm{n}_{\mathrm{Na}} 2.30 \pm 0.02$.

Specimen Data

Mineral and identi- Location

fying number

Brannerite

USNM 96023

Brànnerite

TE 1761-B

Brannerite

Brannerite 1/

USNM 105793

USNM 94286
Ref. no. of Quantity

X-ray patterns

TE 698

USGS-XS

USNM-75 g
Poncia placer,
Boise County, Idaho

$-\infty$

Kelly Gulch, Custer County, Idaho

Kelly Gulch, Custer County, Idaho
Ignited

TE 221

Inv. 2785

Ignited 
Brannerite (Cont.)

1/ Hess, F. I., and Wells, R. C., Franklin Inst. Jour., 189, 225 (1920).

$$
\begin{gathered}
\text { CALCIOSAMARSKITE } \\
(\mathrm{Ca}, \mathrm{Y} \text {, etc. } \mathrm{U}, \mathrm{Th})_{3}(\mathrm{Cb}, \mathrm{Ta}, \mathrm{Fe}, \mathrm{Ti}, \mathrm{Sn})_{5} \mathrm{O}_{15} \\
\text { and } \\
(\mathrm{Ca}, \mathrm{Y}, \text { etc. }, \mathrm{U}, \mathrm{Th}, \mathrm{Zr})_{3}(\mathrm{Cb}, \mathrm{Ta}, \mathrm{Fe}, \mathrm{Ti})_{5} \mathrm{O}_{16} \\
\text { Optics }
\end{gathered}
$$

Brownish and isotropic (metamict) in transmitted light. $n=2.015 \mathrm{I}$ (Hybla), 2.095 (Parry Sound).

\section{Specimen Data}

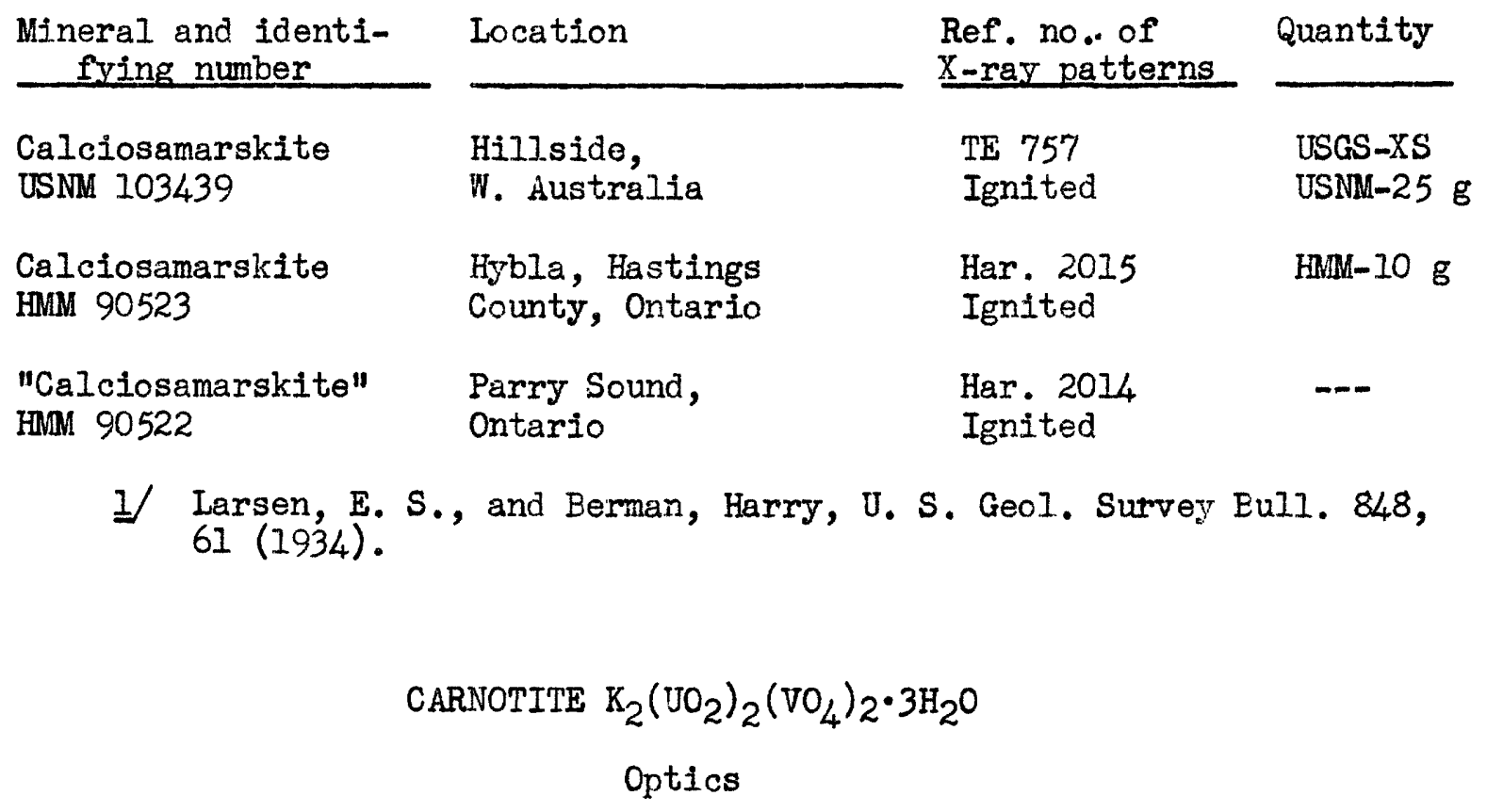

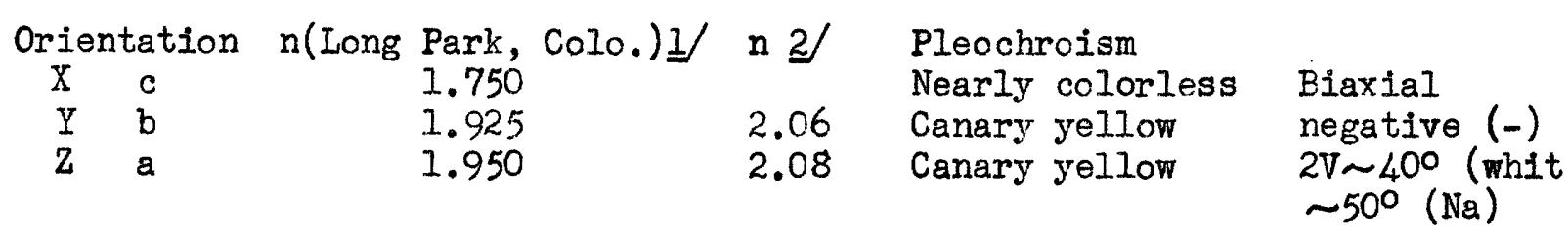


Carnotite (Cont.)

Specimen Data

\begin{tabular}{|c|c|c|c|}
\hline $\begin{array}{l}\text { Mineral and identi- } \\
\text { tying number }\end{array}$ & Iocation & $\begin{array}{l}\text { Ref. no. of } \\
\text { x-rive netterne }\end{array}$ & Quant1ty \\
\hline $\begin{array}{l}\text { Carnotite } \\
\text { PnM } 24675 \\
\text { Phase } 82\end{array}$ & $\begin{array}{l}\text { McIntyre Canyon, } \\
\text { San Miguel County, } \\
\text { Colo. }\end{array}$ & Har. 4224 & BDA-50 E \\
\hline $\begin{array}{l}\text { Carnotite } \\
\text { USN } 90449\end{array}$ & $\begin{array}{l}\text { Mesa County, } \\
\text { Colo. }\end{array}$ & --- & USNMI-100 g \\
\hline $\begin{array}{l}\text { Carnotite } \\
\text { USNI } 92010\end{array}$ & $\begin{array}{l}\text { Gatoway, } \\
\text { Colo. }\end{array}$ & $\ldots$ & $\begin{array}{l}\operatorname{cscos}-x s \\
\operatorname{csN} 4-25 \mathrm{~g}\end{array}$ \\
\hline $\begin{array}{l}\text { Carnotite } \\
\text { USN } 97070\end{array}$ & $\begin{array}{l}\text { Long Park, Montrose } \\
\text { County, Colo. }\end{array}$ & $-\infty$ & $\begin{array}{l}\text { USGS-XS } \\
\text { USNR-300 }\end{array}$ \\
\hline $\begin{array}{l}\text { Carnotite } 3 / \\
\text { USN } 84531\end{array}$ & $\begin{array}{l}\text { Copper Prince mine } \\
\text { Roc Creek, Montrose } \\
\text { County, Colo. }\end{array}$ & $-\infty$ & USM-50 g \\
\hline $\begin{array}{l}\text { Carnotite } 4 / \\
\text { usM } 85710-712\end{array}$ & $\begin{array}{l}\text { Copper Prince claim, } \\
\text { Roc Creek, Montrose } \\
\text { County, Colo. }\end{array}$ & -- & USM-200 g \\
\hline $\begin{array}{l}\text { Carnotite } 5 / \\
\text { IB-LRS/13/48 }\end{array}$ & $\begin{array}{l}\text { Jo Dandy mine, Paradox } \\
\text { Valley, Montrose County, } \\
\text { Colo. }\end{array}$ & $\operatorname{TB} 40$ & USCS-XsC \\
\hline $\begin{array}{l}\text { Carnotite } 5 / \\
\text { TS-IRS/20/48 }\end{array}$ & $\begin{array}{l}\text { Radium No. } 6 \text { mine, Radium } \\
\text { group, San Miguel County, } \\
\text { Colo. }\end{array}$ & in 42 & tscs-Xsc \\
\hline $\begin{array}{l}\text { Carnotite } 5 / \\
\text { TE-LRS } / 25 / 48\end{array}$ & $\begin{array}{l}\text { Colamity No. } 13 \text { mine, } \\
\text { Calamity Mesa, Mesa } \\
\text { County, Colo. }\end{array}$ & $T 243$ & USGS-XSC \\
\hline $\begin{array}{l}\text { Carnotite } 5 / \\
\text { TE-IRS/28/48 }\end{array}$ & $\begin{array}{l}\text { Calamity No. } 13 \text { mine, } \\
\text { Calamity Mesa, Mesa } \\
\text { County, Colo. }\end{array}$ & T2 44 & UsGS-XsC \\
\hline $\begin{array}{l}\text { Carnotite } 5 / \\
\text { TE-IRS/61/48 }\end{array}$ & $\begin{array}{l}\text { Club mine, Uravan } \\
\text { area, Montrose County, } \\
\text { Colo. }\end{array}$ & TE 45 & USCS-XSC \\
\hline $\begin{array}{l}\text { Carnotite } 5 / \\
\text { T2-IRS/62/48 }\end{array}$ & $\begin{array}{l}\text { Club mine, Uravan } \\
\text { area, Montrose County, } \\
\text { Colo. }\end{array}$ & TIE 46 & tses-XsC \\
\hline
\end{tabular}


Carnot1te---Specinen Date (Cont.)

Mineral and Idont1-

Carnot1to

tosin 91286

Carnotite ore TE

Carnotite ore TIS

Carnotite ore

II

Carnotite ore TIE

Carnotite ore TE

Carnotite ore TIE

Carnotite ore TE

Carnotite

Fur(TC-C) Phase 58

Carnotito $5 /$

TE-IRS/ $1 / 48$

Carnotite 2/

USN 95332

USNM R5696

Carnotitie ore TIF
Iocation

Q2ub mine, Uravan area, Nontrose County, Colo.

South olde San M1guel Inv. 4169 Rlver, $16 \mathrm{ml}$ went of Naturita, Colo.

Bald Raglo No. 2 mino, San uiguel County, Colo.

Carbut group, San Juan County, Colo.

Ombar gromp,

San Miguel Oounty, Colo.

Rico mine, San Junn

County, Colo.

Yellow Bird group, Montrose County, Colo.

Gypsum Valley, Colo.

Fall Creek, Colo.

Mauch Chunk,

$\mathrm{Pa}$.

Parco mine, I0llow

Cat group, Thompson area, Grand County, Otah

Cane Springs Pass, $16 \mathrm{mi}$. SE Moab, Otah

Mexican Hat area, San Juan County, Otah
Inv. 4165

of USNM 95332

Rer. no. of

X-rar pattern:

T2 47

USCS-XSC

TSNM-300 8

ves-1t bbl

uscs-1 bbl.

uscs-t bbl.

uses-I bbl.

uses $-3 / 4$ bb]

USCS-3/4 bb:

USCS-1 bbI.

Har. 3943

HIII-9x12, L

TE 38

uscs-Xsc

USNM-21×9,

uscs $-3 / 4$ bb] 
Carnotite--Specimen Data (Cont.)

Mineral and identi- Location fying number

Ref. no. of Quantity X-ray patterns

Carnotite ore

$\mathrm{TE}$

Henry Mountains,

USGS-1 bbl.

Carnotite

USNM 87282

Olary, South

$--$

USGS-X

Australia

USNM-9 $95, M$

Carnotite

$\operatorname{HMM}(\mathrm{TC}-2)$

Olary, South Australia
TE 3069

and Har. 3941

HMMI-6×6,I

If Larsen, E. S., U. S. Geol. Survey Bull. 679, 273 (1921).

2 Hess, F. I., and Foshag, W. F., U. S. Nat. Museun Proc. 72, art. 12 (1927).

3) Hillebrand, W. F., and Ransome, F. L., Am. Jour. Sci, 4th ser., $10,120-144(1.900)$.

4f Hillebrand, W. F., and Ransome, F. I., U. S. Geol. Survey Bull. 262, 9 (1905).

5) Stieff, L. R., Girhard, M. N., and Stern, T. W., U. S. Geol. Survey TEI Rept. 108.

CLARKEITE $\mathrm{UO}_{3} \cdot \mathrm{nH}_{2} \mathrm{O}$ ?

Optics

Orange in transmitted light.

$\begin{array}{lll} & \mathrm{n} & \\ \mathrm{X} & 1.997 & \text { Biaxial negative }(-) \\ \mathrm{Y} & 2.098 & 2 \mathrm{~V} 30^{\circ}-50^{\circ} \\ \mathrm{Z} & 2.108 & r<V, \text { weak }\end{array}$

Specimen Data

\begin{tabular}{|c|c|c|c|}
\hline $\begin{array}{l}\text { Mineral and identi- } \\
\text { fying number }\end{array}$ & Location & $\begin{array}{l}\text { Ref. no. of } \\
\text { X-ray patterns }\end{array}$ & Quantity \\
\hline $\begin{array}{l}\text { Clarkeite } \\
\text { USNM } 96482 \\
\text { Phase } 27\end{array}$ & $\begin{array}{l}\text { Fannie Gouge mine, } \\
\text { Spruce Pine, N. C. }\end{array}$ & $T E 3145$ & USNM $200 \mathrm{~g}$ \\
\hline $\begin{array}{l}\text { Clarkeite } \\
\text { HMM(SLIA) } \\
\text { Phase } 27\end{array}$ & near Spruce Pine, N.C. & Har. 3856 & $\mathrm{MMM}-3 \times 3, \mathrm{M}$ \\
\hline
\end{tabular}


Clarkeite---Specimen Data (Cont.)

Mineral and Identi- Location

Ref. no. of Quantity

fying number

Clarkeite

USNM 103189

Fannie Gouge mine,

X-ray patterns

Clarkeite

USNM C5415

Spruce Pine, N. C.

USGS-XS

USNM-150 g

Spruce Pine, N. C.

USGS-XS

USNM-100 $\mathrm{g}$

Clarkeite I/

Spruce Pine, N. C.

USNM-5 g

USNM 96510

Clarkeite 2/

USNM R6607

Deer Park mine,

Spruce Pine, N. C.

USNM $-5 \mathrm{~g}$

1/ Henderson, E. P., Am. Mineralogist 16, 214 (1931).

2/ Ross, C. S., Henderson, E. P., and Posnjak, E., Am. Mineralogist 16, 213 (1931).

CUPROSKIODOWSKITE $\mathrm{Cu}\left(\mathrm{UO}_{2}\right)_{2} \mathrm{Si}_{2} \mathrm{O}_{7} \cdot 6 \mathrm{H}_{2} \mathrm{O}$

Specimen Data

Mineral and identi- Location

fying number

Cuprosklodowskite

USNM R8383

Cuprosklodowskite

USNM $R 8384$

Cuprosklodowskite

USNM R8391

Cuprosklodowskite

HMM 96587

Phase 11

Bohemia
Ref. no. of Quantity X-ray patterns

TE 297

USGS $-X S$

USNM $-9 \times 9,11$

Belgial. longo

-.- USGS $X S$

USMM-30 $\mathrm{g}$

USGS $=X S$

USMM $-6 \times 9, M$

Har. 3835

HMM- $3 \times 9, \mathrm{~L}$

Uranochalcite--A hydrous, copper, uranium, and calcium sulfate, of questionable validity.

Uranochalcite?

HMM 807

Phase 67
Joachimsthal,

Bohemia
Har. 1630

HMM $-3 \times 6, \mathrm{~L}$ 
Uranochalcite---Specimen Data (Cont.)

\begin{tabular}{|c|c|c|c|}
\hline $\begin{array}{l}\text { Mineral and identi- } \\
\text { fying number }\end{array}$ & Location & $\begin{array}{l}\text { Ref. no. of } \\
\text { X-ray patterns }\end{array}$ & Quantity \\
\hline $\begin{array}{l}\text { Uranochalcite? } \\
\text { HMM } 807 \text { Phase } 73\end{array}$ & $\begin{array}{l}\text { Joachimsthal, } \\
\text { Bohemia }\end{array}$ & Har. 1631 & $\mathrm{HMM}-3 \times 6, \mathrm{~L}$ \\
\hline $\begin{array}{l}\text { Uranochalcite? } \\
\text { AMNH } 19156\end{array}$ & $\begin{array}{l}\text { Joa chims thal, } \\
\text { Bohemia }\end{array}$ & $-\cdots$ & $\mathrm{HMM}-3 \times 6, \mathrm{I}$ \\
\hline
\end{tabular}

Voglianite--A hydrous calcium and uranium sulfate, of questionable validity. The type material of Vogl for uranochalcite and voglianite has not been found. Present museum specimens bearing these names have proved to be cuprosklodowskit

Voglianite?

Joachimsthal,

$\operatorname{man}-6 \times 6, \mathrm{~L}$

AMNH 16824

Bohemia

CURITE $\mathrm{Pb}_{2} \mathrm{U}_{5} \mathrm{O}_{17} \cdot 4 \mathrm{H}_{2} \mathrm{O}$ ?

Optics

In transmitted light, yellow to reddish orange.

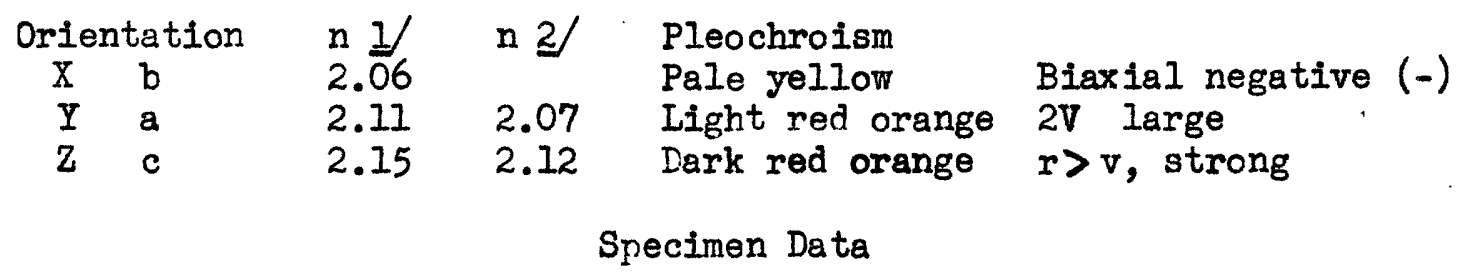

Mineral and identifying number

Curite

USNM $\mathrm{C} 4512$

Phase 49

Curite

USNM C4513

Curite

JSSNM 94711

Curite

JJSM 94713
Location

Chinkolobwe mine,

Kasola, Katanga,

Belgian Congo

Chinkolobwe mine,

Kasola, Katanga,

Belgian Congo

Kasola mine,

Belgian Congo

Kasola mine,

Belgian Congo
Ref. no. of X-ray patterns

TE 3142

Quantity

USNM-100 g

USGS-XS

USNM-100 g

USGS-XS

USNM-100 g

USGS-XS 
Curite---Specimen Data (Cont.)

\begin{tabular}{|c|c|c|c|}
\hline $\begin{array}{l}\text { Mineral and identi- } \\
\text { fying number }\end{array}$ & Location & $\begin{array}{l}\text { Ref. no. of } \\
\text { X-ray patterns } \\
\end{array}$ & Quantity \\
\hline $\begin{array}{l}\text { Curite } \\
\text { USNM } 95601\end{array}$ & $\begin{array}{l}\text { Chinkolobwe mine, } \\
\text { Belgian Congo }\end{array}$ & -- & $\begin{array}{l}\text { USGS-XS } \\
\text { USNM-200 }\end{array}$ \\
\hline $\begin{array}{l}\text { Curite } \\
\text { USNM } 95603\end{array}$ & $\begin{array}{l}\text { Katanga, Belgian } \\
\text { Congo }\end{array}$ & -- & $\begin{array}{l}\text { USGS-XS } \\
\text { USNM-200 g }\end{array}$ \\
\hline $\begin{array}{l}\text { Curite? } \\
\text { USNM } 104784\end{array}$ & $\begin{array}{l}\text { Chinkolobwe, } \\
\text { Belgian Congo }\end{array}$ & --- & $\begin{array}{l}\text { USGS-XS } \\
\text { USNM-75 g }\end{array}$ \\
\hline $\begin{array}{l}\text { Curite } \\
\text { USNM R5896 }\end{array}$ & $\begin{array}{l}\text { Chinkolobwe mine, } \\
\text { Kasola, Katanga, } \\
\text { Belgian Congo }\end{array}$ & -- & $\begin{array}{l}\text { USGS-XS } \\
\text { USNM-100 g }\end{array}$ \\
\hline $\begin{array}{l}\text { Curite } \\
\text { USNM R590I }\end{array}$ & $\begin{array}{l}\text { Chinkolobwe mine, } \\
\text { Kasola, Ka tanga, } \\
\text { Belgian Congo }\end{array}$ & $-\infty-$ & $\begin{array}{l}\text { USGS-XS } \\
\text { USNM-100 g }\end{array}$ \\
\hline $\begin{array}{l}\text { Curite } \\
\text { HMM Phase } 49\end{array}$ & $\begin{array}{l}\text { Katanga, Belgian } \\
\text { Congo }\end{array}$ & Har. 1129 & --- \\
\hline $\begin{array}{l}\text { Curite } \\
\text { HMM } 91181\end{array}$ & $\begin{array}{l}\text { Wälsendorf, } \\
\text { Bavaria }\end{array}$ & Har. 3921 & USGS- $3 \times 9, I$ \\
\hline
\end{tabular}

Phase 50

1/ Larsen, E. S., and Berman, Harry, U. S. Geol. Survey Bull. 848, 209 (1934).

2) Billiet, V., Sos. frang. minéralogie Bull. 49, 136 (1925).

$$
\begin{gathered}
\text { DELORENZITE }\left(\mathrm{Y}, \mathrm{U}, \mathrm{Fe}^{2}\right)(\mathrm{Ti}, \mathrm{Sn})_{3} \mathrm{O}_{8} ? \\
\text { Optics }
\end{gathered}
$$

In transmitted light, chestnut brown. Metamict and isotropic.

\begin{tabular}{|c|c|c|c|}
\hline $\begin{array}{l}\text { Mineral and identi- } \\
\text { fying number }\end{array}$ & Location & $\begin{array}{l}\text { Ref. no. of } \\
\text { X-ray patterns }\end{array}$ & Quantity \\
\hline $\begin{array}{l}\text { Delorenzite } \\
\text { HMM } 89594\end{array}$ & $\begin{array}{l}\text { Val Vigesso } \\
\text { Italy }\end{array}$ & $\begin{array}{l}\text { Har. } 11350 \\
\text { Ignited } \\
\text { Har. } 11307 \\
\text { Ignited, faint }\end{array}$ & $\begin{array}{c}--- \\
\text { atterns }\end{array}$ \\
\hline
\end{tabular}
Specimen Data 
DEWINDTITE $\mathrm{Pb}_{3}\left(\mathrm{UO}_{2}\right)_{5}\left(\mathrm{PO}_{4}\right)_{4}(\mathrm{OH})_{4} \cdot 10 \mathrm{H}_{2} \mathrm{O}$

\section{Optics I}

Not pleochroic.

Orientation

$x \quad b$

Y $c$

Z a

$$
\begin{aligned}
& n \\
& 1.762 \\
& 1.763
\end{aligned}
$$

\begin{tabular}{|c|c|c|c|}
\hline $\begin{array}{l}\text { Mineral and identi- } \\
\text { fying number }\end{array}$ & Iocation & $\begin{array}{l}\text { Ref. no. of } \\
\text { X-ray patterns }\end{array}$ & Quantity \\
\hline $\begin{array}{l}\text { Dewindtite } \\
\text { USNM } 95605\end{array}$ & $\begin{array}{l}\text { Chinkolobwe, Katanga, } \\
\text { Belgian Congo }\end{array}$ & $\mathrm{TE} 3137$ & $\begin{array}{l}\text { USGS-XS } \\
\text { USNM-25 g }\end{array}$ \\
\hline $\begin{array}{l}\text { Dewindtite } \\
\text { USNM C4513 }\end{array}$ & $\begin{array}{l}\text { Chinkolobwe, Katanga, } \\
\text { Belgian Congo }\end{array}$ & $-\infty$ & $\begin{array}{l}\text { USGS-XS } \\
\text { USNM-100 g }\end{array}$ \\
\hline $\begin{array}{l}\text { Dewindtite } \\
\text { USNM C4516 }\end{array}$ & $\begin{array}{l}\text { Chinkolobwe, Katanga, } \\
\text { Belgian Congo }\end{array}$ & --- & $\begin{array}{l}\text { USGS-XS } \\
\text { USMM-50 g }\end{array}$ \\
\hline $\begin{array}{l}\text { Dewindtite } \\
\text { USNM R5901 }\end{array}$ & $\begin{array}{l}\text { Chinkolobwe, Katanga, } \\
\text { Belgian Congo }\end{array}$ & --- & $\begin{array}{l}\text { USGS-XS } \\
\text { USMM-100 g }\end{array}$ \\
\hline $\begin{array}{l}\text { Dewindtite } \\
\text { HMM W-1 } \\
\text { Phase } 46\end{array}$ & $\begin{array}{l}\text { Wäisendorf, } \\
\text { Bavaria }\end{array}$ & --- & -- \\
\hline
\end{tabular}

Specimen Data

If Schoep, Alfred, Soc. franç. minéralogie Bull. 48, 77 (1925).

$$
\begin{gathered}
\text { DJaLMaITE }(\mathrm{U}, \mathrm{Ca}, \mathrm{Pb})(\mathrm{Ta}, \mathrm{Cb}, \mathrm{Ti})_{3} \mathrm{O}_{9} \cdot \mathrm{nH}_{2} \mathrm{O} \\
\text { Optics }
\end{gathered}
$$

Transparent in thin splinters, with a yellowish-brown color $n=1.97$ Specimen Data

Mineral and identi- Location fying number

Djalmaite

USNM 105973
Volta Grande deposit, Nazareno district, São Jožo del Rei, Minas Geraes, Brazil
Biaxial positive (t) $r<v$

2E large 


$$
\text { DUMONTITE } \mathrm{Pb}_{2}\left(\mathrm{OO}_{2}\right)_{3}\left(\mathrm{PO}_{4}\right)_{2}(\mathrm{OH})_{4} \cdot 3 \mathrm{H}_{2} \mathrm{O}
$$

Optics

$\begin{array}{ccc}\text { Orientation } & n \\ \bar{X} & \mathrm{a} & 1.88 \\ \mathrm{Y} & \mathrm{c} & \\ \mathrm{Z} & \mathrm{b} & 1.89\end{array}$

Pleochroism

Pale yellow

Deep yellow

Biaxial psoitive $(t)$

$r<v$

2V large

Specimen Data

Mineral and identi-
fying number
Dumontite
USNM 95884
Dumontite
USNM 97140
Dumontite
USNM R6701

Location

Ref. no. of

Quantity

$X$-ray patterns

Chinkolcbwe, Katanga, TE 3169

Belgian Congo

Chinkolobwe, Ka tanga,

$--$

USGS-XS

Belgian Congo

USNM-6×6,M

Chinkolobwe, Katanga,

USGS-XS

Belgian Congo

USNM- $10 \times 12, I$

EPIIANTHINITE $\mathrm{yOO}_{3} \cdot \mathrm{xH}_{2} \mathrm{O}$ ?

Optics I

\begin{tabular}{cll} 
Orientation & \multicolumn{1}{c}{$\mathrm{n}$} \\
$\mathrm{X}$ & $\mathrm{c}$ & 1.70 \\
$\mathrm{Y}$ & $\mathrm{b}$ & 1.79 \\
$\mathrm{Z}$ & $\mathrm{a}$ & 1.793
\end{tabular}

Pleochroism

Pale yellow

Yellow

Deep yellow

Biaxial negative (-) $2 V$ small

Specimen Data

\begin{tabular}{|c|c|c|c|}
\hline $\begin{array}{l}\text { Mineral and identi- } \\
\text { fying number }\end{array}$ & Location & $\begin{array}{l}\text { Ref. no. of } \\
\text { X-ray patterns }\end{array}$ & Quantity \\
\hline $\begin{array}{l}\text { Epiianthinite? } \\
\text { USNM R8378 }\end{array}$ & $\begin{array}{l}\text { Katanga, Belgian } \\
\text { Congo }\end{array}$ & -- & $\begin{array}{l}\text { USGS-X } \\
\text { USNM- } 9 \times 12, \mathrm{~L}\end{array}$ \\
\hline $\begin{array}{l}\text { Ep.ianthinite? } \\
\text { USNM R8368 }\end{array}$ & $\begin{array}{l}\text { Katanga, Belgian } \\
\text { Congo }\end{array}$ & -.. & $\begin{array}{l}\text { USGS-XS } \\
\text { USNM- } 15 \times 21,\end{array}$ \\
\hline
\end{tabular}

I/ Schoep, Alfred, and Stradiot, Sadi, Am. Mineralogist 32, 344-350 (1947). 


$$
\text { ISCHENITE ( } \mathrm{Ce}, \mathrm{Ca}, \mathrm{Ie} \text {, Th })(\mathrm{TI}, \mathrm{Cb})_{2} \mathrm{O}_{6}
$$

Optios

Isotropic (metamict) and transmits light in only the thinnest splinters. Reddish brown to light brown. Beoomes anisotropio on ignition, and the refractive index increases somenhat.1/ $n=2.26 \pm 0.01,2 / \mathrm{ln}-$ creasing to 2.285 on ignition.

\section{Specimen Date}

Hineral and Identifring number

Eschynite

BuB 87857

Eschynite

BDin 87859

Ischynite

han 58082

Bschynite

USNM 94581

Es chynite

HNA 84641
Iocation

Statoust,

Urals

Miask, Orals

Ilftterö, Norway

Hitterö

Norway

Saetersdalen, Norway
Ref. no. of X-rer patterns

Bar. 2016

Ifnited

Har. 1872

Igntted

Hlar. 1912

Ignited

T2 24

Ignited

Har. 1853

Ignited
Quantity
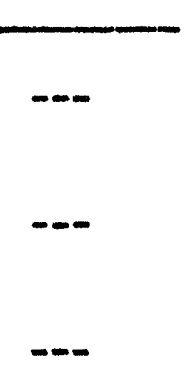

USGS-XS

USNM-30 g

1/ Larsen, E. S., and Berman, Barry, U. S. Geol. Survey Bull. 848, 65 (1934).

2/ Larsen; E. S., U. S. Geol. Survey Bull. 679, 72 (1921).

$$
\text { BUXENITE }(\mathrm{I}, \mathrm{Ca}, \mathrm{Ce}, \mathrm{U}, \mathrm{Th})(\mathrm{Cb}, \mathrm{Ta}, \mathrm{Ts})_{2} \mathrm{O}_{6}
$$

\section{Optice 1}

In transmitted light, brown, yellow brown, or reddish brown. Isotropic (metamict). The refractive index increases after ignition.

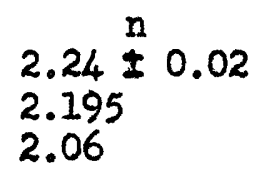

$2.24 \pm 0.02$

2.06
(Hitterö, Norway)

(no locality; 2.23 after ignition) (no locality; 2.22 after ignition) 
Euxenite (Cont.)

\section{Specimen Data}

\section{Mineral and identi- fying number}

Euxenite

IIE

Euxenite

HMM

Euxenite USNM 97694

Euxenite HMM

Euxenite

HMM 90791

Euxenite

USNM 103025

Euxenite

HMM 8.4648

Lyndochite

HMM 90520

Lyndochite

USNM 102745
Location

-..

Colorado

from A. C. Lane

Castello Branco,

Fazenda de Sta. Clara,

$11 \mathrm{kms}$. from Tocantens

Pomba, Minas Geraes, Brazil

Brazil

South Sherbrooke

Township, Lanark

County, Ontario

Ref. no. of

X-ray patterns

TE 26

Har. 2275

Ignited

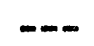

USGS-XS

USMM-150 g
Har. 9545

Ignited.

Har. 9555

Ignited

Har. 1710

Ignited

$3 \mathrm{hrs}$. to

red heat

Lot 12, Con. 5, South

USGS-XS

Sherbrooke Township,

Ontario.

USNM-300 g

Tangen Quarry, near

Kragerö, Norway

Har. 2029

Ignited

HMM-100 g

Lyndochite--Variety of euxenite

Lyndoch Township,

Ontario

Har. 1926

Ignited

Lyndoch Township,

Ontario
TE 748

Ignited
HMM-5 g

USGS-XS

USNM-20 $\mathrm{g}$

Tanteuxenite--Variety of euxenite

Tanteuxenite

USNM 96284

Tanteuxenite

HMM 90701
Wleys, West

Australia

Pilbara Goldfield,

West Australia
TE 653

USGS-XS

USNM-10 $\mathrm{g}$ 
Euxenite---Specimen Data (Cont.)

Toddite--Possibly a mixture of columbite and euxenite

Mineral and identi- Location

fying number

Toddite

HMM 90526 Ontario
Ref. no. of

X-ray patterns

Har. 334

I) Larsen, E. S., U. S. Geol. Survey BuIl. 679, 73 (1921); and Larsen, E. S., and Berman, Harry, U. S. Geol. Survey Bull. $848,62,63,64$ (1934).

Dill Township,

Quantity

FERGUSONITE ( $\mathrm{Y}, \mathrm{Er}, \mathrm{Ce}, \mathrm{Fe})(\mathrm{Cb}, \mathrm{Ta}, \mathrm{Ti}) \mathrm{O}_{4}$

Optics

In transmitted light, light brown to dark brown, and some specimens cloudy. The metamict material is isotropic. On ignition it becomes anisotropic but in such fine grain as to permat measurement only of the mean index of refraction. I/ Large ignited grains, however, may exhibit birefringence possibly due to strain. I/ Refractive indices:

$\begin{array}{llc}\text { Baringer Hill, Texas 2/ } & \text { Metamict } & \begin{array}{c}\text { Ignited } \\ \text { (mean index) }\end{array} \\ \text { Boksput, Africa 3/ } & 2.19 \pm 0.02 & \\ \text { Risör, Norway 4J } & 2.05 & \\ \text { No locality 4/ } & 2.08 & 2.07 \\ \text { No locality } & 2.115 & 2.070 \\ \text { Risör, Norway } & 2.175 & 2.142 \\ \text { Virginia } & 2.08 & 2.08 \\ & 2.06 & 2.06\end{array}$

Anisotropic crystals with an isotropic (metamict) shell have been found at Hundholmen, Norway.5/ Uniaxial, negative (-), with strong birefringence and weak pleochroism, $0>\mathrm{E}$.

\section{Specimen Data}

\begin{tabular}{|c|c|c|c|}
\hline $\begin{array}{l}\text { Mineral and identi- } \\
\text { fying number }\end{array}$ & Locątion & $\begin{array}{l}\text { Ref. no. of } \\
X \text {-ray patterns }\end{array}$ & Quantity \\
\hline $\begin{array}{l}\text { Fergusonite } \\
\text { HMM } 81181\end{array}$ & Petaca, N. Mex. & $\begin{array}{l}\text { Har. } 2031 \\
\text { Ignited } \\
\text { Har. } 2030 \\
\text { Ignited }\end{array}$ & $-\cdots$ \\
\hline
\end{tabular}


Fergusonite---Specimen Data (Cont.)

\begin{tabular}{|c|c|c|c|}
\hline $\begin{array}{l}\text { Mineral and identi- } \\
\text { fying number }\end{array}$ & Location & $\begin{array}{l}\text { Ref. no. of } \\
\text { X-ray patterns }\end{array}$ & Quantity \\
\hline $\begin{array}{l}\text { Fergusonite } \\
\text { USNM } 80705\end{array}$ & $\begin{array}{l}\text { Spruce Pine, } \\
\text { Mitchell County, N.C. }\end{array}$ & $\begin{array}{l}\text { TE } 745 \\
\text { Ignited }\end{array}$ & $\begin{array}{l}\text { USGS-XS } \\
\text { USNM-30 g }\end{array}$ \\
\hline $\begin{array}{l}\text { Fergusonite } \\
\text { USNM R5878 }\end{array}$ & $\begin{array}{l}\text { Llano County, } \\
\text { Texas }\end{array}$ & -- & $\begin{array}{l}\text { USGS-XS } \\
\text { USNM-50 g }\end{array}$ \\
\hline $\begin{array}{l}\text { Fergusonite } \\
\text { USNM C } 4510\end{array}$ & $\begin{array}{l}\text { Llano County, } \\
\text { Texas }\end{array}$ & $\begin{array}{l}\text { TE } 727 \\
\text { Ignited }\end{array}$ & $\begin{array}{l}\text { USGS-XS } \\
\text { USNM-100 g }\end{array}$ \\
\hline $\begin{array}{l}\text { Fergusonite } \\
\text { HMM } 87817\end{array}$ & $\begin{array}{l}\text { Rulandsdalens, near } \\
\text { Risör, Norway }\end{array}$ & Har. 1838 & -- \\
\hline $\begin{array}{l}\text { Fergusonite } \\
\text { HMM } 58254\end{array}$ & $\begin{array}{l}\text { Ytterby, } \\
\text { Sweden }\end{array}$ & $\begin{array}{l}\text { Har. } 1910 \\
\text { Ignited }\end{array}$ & --- \\
\hline
\end{tabular}

Adelpholite--Synonym of fergusonite? A poorly defined substance, possibly an altered mossite.

Adelpholite

USNM R5058

Tammela, Finland

$--$

Arrhenite--An altered fergusonite

Arrhenite

USNM R5135

Ytterby,

Sweden

$--$

USGS-XS

USNAL-25 g

Bragite--Synonym of fergusonite

Bragite

USNM R505I

Arendal,

Norway

USGS-XS

USNM-10 $\mathrm{g}$

Risörite--Synonym of ergusonite

Risörite

USNM 93038

Risör,

Norway

$-\infty$

USGS-XS

USNM-50 g

Rutherfordite--An altered fergusonite

Rutherfordite USNM R5054
Rutherford County,

N. C.
USGS-XS

USNM-150 g 
Fergusonite----Specimen Data (Cont.)

\section{Sipylite--Synonym of fergusonite}

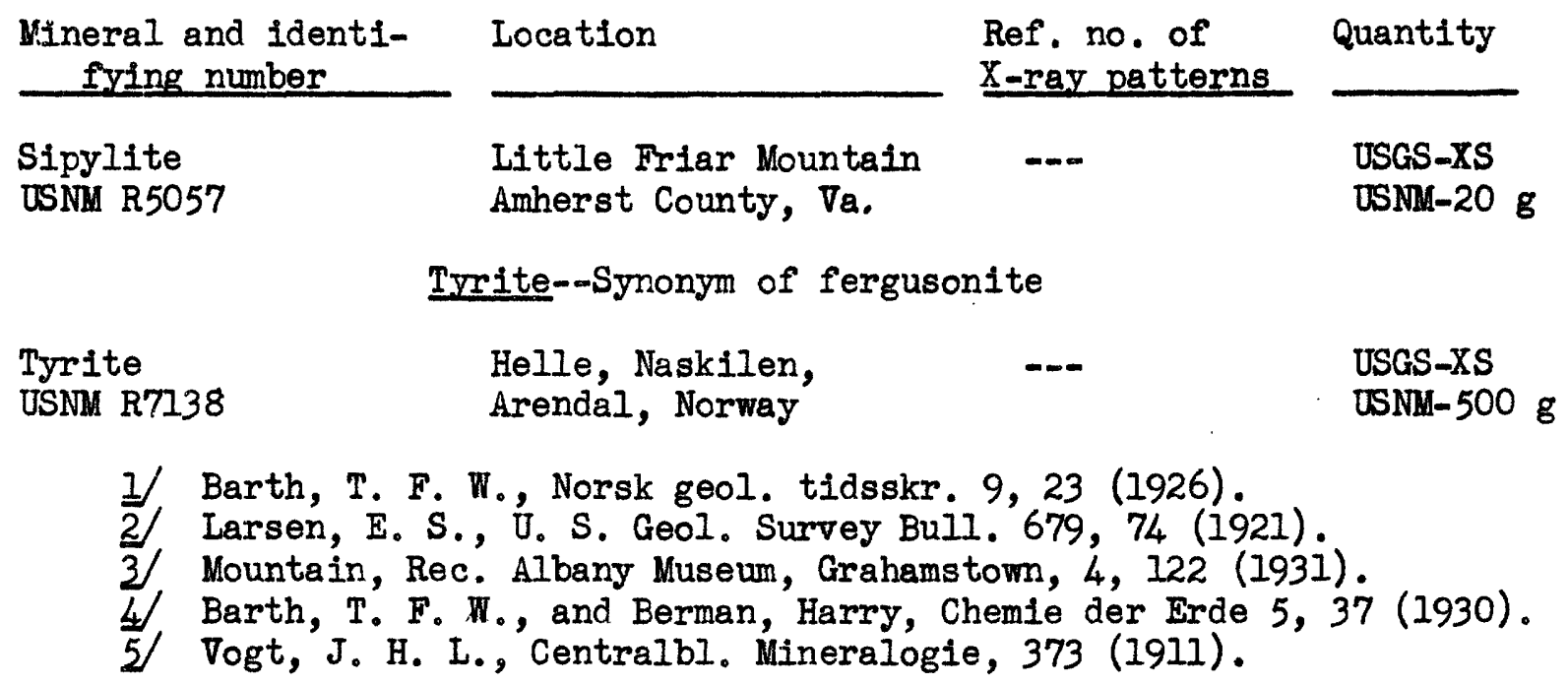

FORMANITE $(\mathrm{U}, \mathrm{Th}, \mathrm{Ca})(\mathrm{Ta}, \mathrm{Cb}, \mathrm{Ti}) \mathrm{O}_{4}$

Specimen Data

\begin{tabular}{|c|c|c|c|}
\hline $\begin{array}{l}\text { Mineral and identi- } \\
\text { fying number }\end{array}$ & Location & $\begin{array}{l}\text { Ref. no. of } \\
\text { X-rar patterns }\end{array}$ & Quantity \\
\hline $\begin{array}{l}\text { Formanite } \\
\text { HMM } 8614 \mathrm{~A}\end{array}$ & $\cdots$ & $\begin{array}{l}\text { Har. } 2850 \\
\text { Ignited }\end{array}$ & -- \\
\hline $\begin{array}{l}\text { Formanite } \\
\text { HMM 861 } \mathrm{B}\end{array}$ & -- & $\begin{array}{l}\text { Har. unnumbered } \\
\text { Ignited }\end{array}$ & -- \\
\hline
\end{tabular}

FOURMARIERITE $\mathrm{PbU}_{4} \mathrm{O}_{13} \cdot 5 \mathrm{H}_{2} \mathrm{O}$ ?

Optics I/

In transmitted light, yellow or reddish yellow. Pleochroic in shades of yellow and orange.

$\begin{array}{clll}\text { Orientation } & n & \text { Pleochroism } & \\ \text { X c } & 1.85 & \text { Colorless } & \text { Blaxial negative }(-) \\ \text { Y b } & 1.92 & \text { Pale yellow } & \text { 2V large } \\ \text { Z a } & 1.94 & \text { Yellow } & r>v \text {, strong }\end{array}$


Fourmarierite (Cont.)

Specimen Data

Mineral and identi- Location fying number

Fourmarierite

USNM R5899
Ref. no. of X-rat patterns

TE 601
Quantity

USGS-XS

USNMM-15 g

1 Larsen, E. S., and Berman, Harry, U. S. Geol. Survey Bul1. 848, 205 (1934), and Billiet, v., Soc. franç. minéralogie Bull. 49, 136 (1926)'.

FRITZSCHEITE $\mathrm{Mn}\left(\mathrm{UO}_{2}\right)_{2}\left[(\mathrm{P}, \mathrm{V}) \mathrm{O}_{4}\right]_{2} \cdot 8 \mathrm{H}_{2} \mathrm{O} ?$

Specimen Data

Mineral and identi-
fying number

Fritzscheite

USNM R5685
Iocation

Johanngeorgenstadt, Saxony
Ref. no. of X-ray patterns

Quantity

USGS-X

USNM-6x6,I

\section{GUMMITE $\mathrm{UO}_{3} \cdot \mathrm{nH}_{2} \mathrm{O}$}

Optics

In transmitted light, orange or yellow. Anisotropic; some is very finely divided and seemingly isotropic. The indices vary widely: $n$. $1.575 \mathrm{I} /$ (Easton, Pa.), $1.96 \mathrm{2}$ (Kambowe, Katanga). Gummite from Spruce Pine, N. . C., $3 /$ is biaxial negative $(-)$ with $2 \mathrm{~V}=60^{\circ}$ and $n \mathrm{X}=1.742$, $n \mathrm{Y}=$ $1.762, \mathrm{nZ}=1.776$, and not pleochroic.

\section{Specimen Data}

\begin{tabular}{|c|c|c|c|}
\hline $\begin{array}{l}\text { Mineral and identi- } \\
\text { fying number }\end{array}$ & Location & $\begin{array}{l}\text { Ref. no. of } \\
\text { X-Ray patterns }\end{array}$ & Quantity \\
\hline $\begin{array}{l}\text { Gummite } \\
\text { USNM } 102618\end{array}$ & $\begin{array}{l}\text { Ruggles mine, } \\
\text { Grafton, N.H. }\end{array}$ & --- & $\begin{array}{l}\text { USGS-XS } \\
\text { USNM-100 g }\end{array}$ \\
\hline $\begin{array}{l}\text { Gummite } \\
\text { HMM H-I, Phase } 26\end{array}$ & $\begin{array}{l}\text { Ruggles mine, } \\
\text { Grafton, N.H. }\end{array}$ & Har. 3843 & --- \\
\hline
\end{tabular}


Gummite---Specimen Data (Cont.)

\begin{tabular}{|c|c|c|c|}
\hline $\begin{array}{l}\text { Mineral and identi- } \\
\text { fying number }\end{array}$ & Location & $\begin{array}{l}\text { Ref. no. of } \\
\text { X-ray patterns }\end{array}$ & Quantity \\
\hline $\begin{array}{l}\text { Gummite } \\
\text { USNM } 96482\end{array}$ & $\begin{array}{l}\text { Spruce Pine, } \\
\text { N.C. }\end{array}$ & TE 3146 & USNM-200 g \\
\hline $\begin{array}{l}\text { Gummite } \\
\text { USNM } 103189\end{array}$ & $\begin{array}{l}\text { Fannie Gouge mine, } \\
\text { Spruce Pine, N.C. }\end{array}$ & -- & $\begin{array}{l}\text { USGS-XS } \\
\text { USNM-200 g }\end{array}$ \\
\hline $\begin{array}{l}\text { Gummite } \\
\text { USNM C } 5415\end{array}$ & $\begin{array}{l}\text { Spruce Pine, } \\
\text { N.C. }\end{array}$ & -- & $\begin{array}{l}\text { USGS-XS } \\
\text { USNM-100 } \mathrm{g}\end{array}$ \\
\hline $\begin{array}{l}\text { Gummite } 3 / \\
\text { USNM } 86120\end{array}$ & $\begin{array}{l}\text { Wiseman mica mine, } \\
\text { Mitchell County, N.C. }\end{array}$ & -- & USNM-10 g \\
\hline $\begin{array}{l}\text { Gummite? } \\
\text { USNM } 104784\end{array}$ & $\begin{array}{l}\text { Chinkolobwe, Katanga, } \\
\text { Belgian Congo }\end{array}$ & -- & $\begin{array}{l}\text { USGS-XS } \\
\text { USNM-500 g }\end{array}$ \\
\hline $\begin{array}{l}\text { Gummite } \\
\text { USNM R8384 }\end{array}$ & $\begin{array}{l}\text { Kambowe, Katanga, } \\
\text { Belgian Congo }\end{array}$ & $-\infty$ & $\begin{array}{l}\text { USGS-XS } \\
\text { USNM-50 g }\end{array}$ \\
\hline $\begin{array}{l}\text { Gummite } \\
\text { USNM R8397 }\end{array}$ & $\begin{array}{l}\text { Luiswishi, Katanga } \\
\text { Belgian Cóngo }\end{array}$ & -- & $\begin{array}{l}\text { USGS-XS } \\
\text { USNM-50 g }\end{array}$ \\
\hline $\begin{array}{l}\text { Gummite } \\
\text { USNM } 91225\end{array}$ & $\begin{array}{l}\text { Brejaúba, Minas } \\
\text { Geraes, Brazil }\end{array}$ & -- & USGS-XS \\
\hline $\begin{array}{l}\text { Gummite } \\
\text { HMM Phase } 31\end{array}$ & $\begin{array}{l}\text { Gordonia, South } \\
\text { Africa }\end{array}$ & Har. 3967 & HMM-10 $\mathrm{g}$ \\
\hline $\begin{array}{l}\text { Gummite (red zone) } \\
\text { AMNH } \\
\text { Phase } 24\end{array}$ & Rajputana, India & Har. 4089 & $-\infty$ \\
\hline $\begin{array}{l}\text { Gummite (brown zone) } \\
\text { AMNH } \\
\text { Phase } 29\end{array}$ & Rajputana, India & Har. 4087 & $-\infty$ \\
\hline $\begin{array}{l}\text { Gummite } \\
\text { HMM Phase } 28\end{array}$ & -- & Har. 4125 & $-\infty$ \\
\hline
\end{tabular}

Eliasite--Synonym of gummite

Eliasite

USNM R5888

Ellasite

USNM C2257
Joachimsthal,

Bohemia

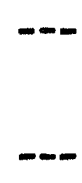

Joachims thal,
USGS-XS

USNA-50 g

Bohemia
USGS-XS

USNM-10 $\mathrm{g}$ 
Gummite---Specimen Data (Cont.)

Pittinite--Synonym of gummite

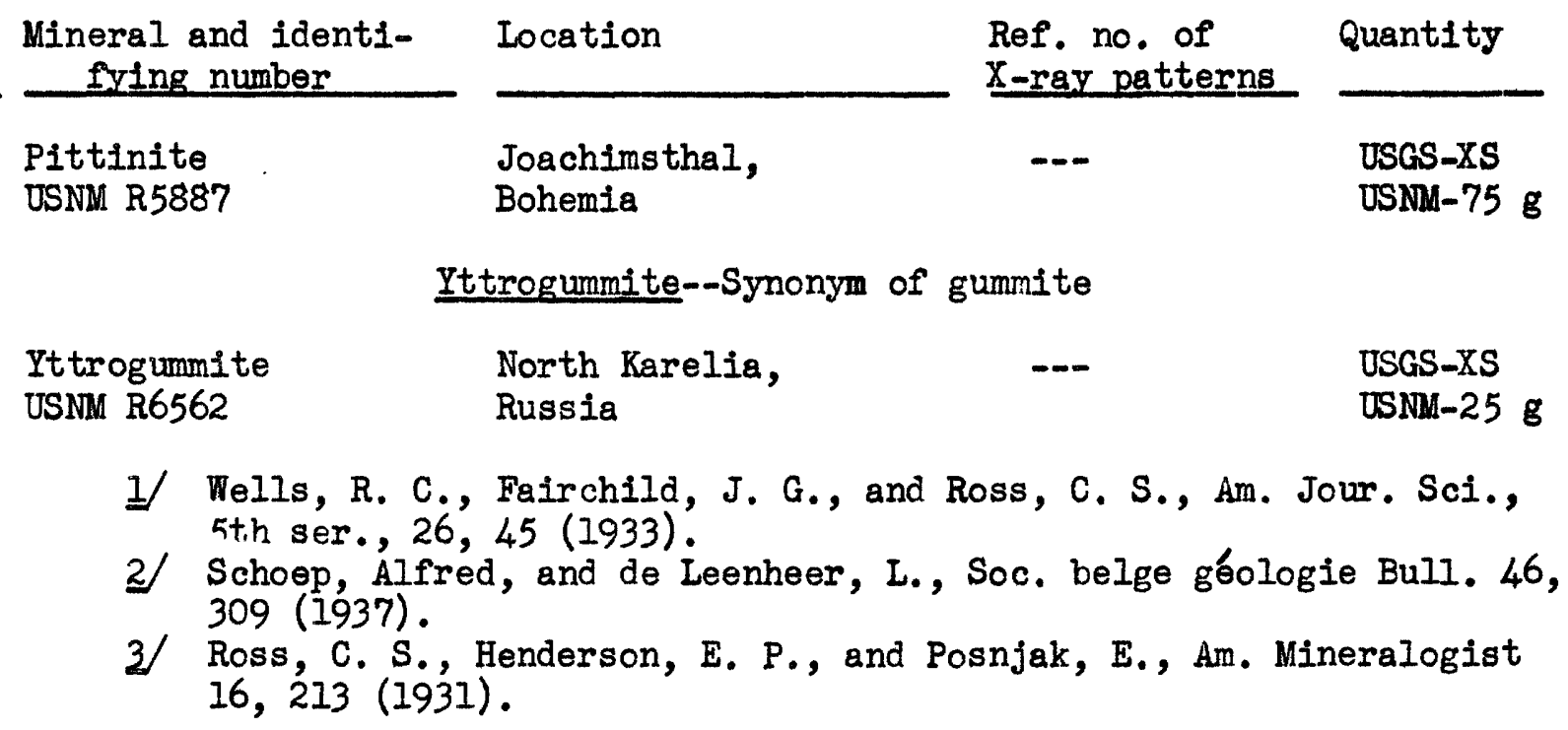

X-ray patterns

Quantity

USGS-XS USNM-75 $\mathrm{g}$

\section{Yttrogummite--Synonym of gummite}

Yttrogummite USNM R6562

North Karelia, Russia

USGS-XS

USNM-25 g

I/ Wells, R. C., Fairchild, J. G., and Ross, C. S., Am. Jour. Sci., 5t.h ser., 26, 45 (1933).

2) Schoep, Alfred, and de Leenheer, L., Soc. belge géologie Bull. 46, 309 (1937).

3/ Ross, C. S., Henderson, E. P., and Posnjak, E., Am. Mineralogist 16,213 (1931).

IANTHINITE $2 \mathrm{OO}_{2} \cdot 7 \mathrm{H}_{2} \mathrm{O}$ ?

Optics

In transmitted light, dark violet.

\begin{tabular}{clll} 
Orientation & \multicolumn{1}{c}{$\mathrm{n} I /$} & Pleochroism & \\
$\mathrm{X}$ c & $1.674 \pm 0.003$ & Colorless & \\
I b & $1.90 \pm 0.02$ & Violet & Biaxial negative (-) \\
Z a & $1.92 \pm 0.02$ & Dark violet &
\end{tabular}

Specimen Data

Mineral and identi- Location fying number

Ianthinite

HMM 87090

Phase 6

Ianthinite

USNM 95990

Katanga,

Katanga,
Ref. no. of

X-ray patterns

Har. 4214

Belgian Congo

Belgian Congo
Quantity

HMM-3×3,L

USNM- $1 \times], I$

I) Billiet, V., Soc. frang. minéralogie Bull. 49, 136 (1926). 
ISHIKAWAITE (U, $\mathrm{Fe}, \mathrm{Y}$, etc. $)(\mathrm{Cb}, \mathrm{Ta}) \mathrm{O}_{4}$

Specimen Data

Mineral and identi-
fying number

Ishikawaite?

USNM 105862
Location

Ishikawa, Fukushima, Japan
Ref. no. of

X-ray patterns

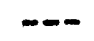

Quantity

USGS-X

USNM-3 $g$

\section{JOHANN ITE $\mathrm{Cu}\left(\mathrm{UO}_{2}\right)_{2}\left(\mathrm{SO}_{4}\right)_{2}(\mathrm{OH})_{2} \cdot 6 \mathrm{H}_{2} \mathrm{O}$ \\ Optics}

Strongly pleochroic, with X colorless, $Y$ pale yellow, $Z$ greenish yellow or canary jellow. Biaxial.

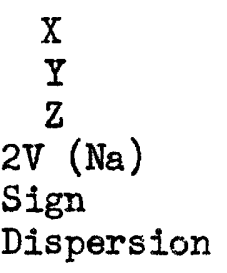

n Joachimsthal I/

1.595

1.614

$\sim 90^{\circ}$

$(-)$

$r>v$, strong

Specimen Data
Mineral and identifying number

Johannite

USNM R6329

Johannite

USNM C4763

Johannite

HMM 89613

Phase 3

Johannite

HMM 68991

Gilpinite USNM C4766
Location

Wood mine, Gilpin

County, Colo.

Joa chims thal,

Bohemia

Joachims thal,

Bohemia

Ref. no. of

X-ray patterns

Joachins thal,

Bohemia

Gilpinite $=$ johannite

Montrose County,

$-\infty$

Quantity

USGS-XS

USNM- $3 \times 3, L$

TE 298

USGS -XS

USNM-6x 6, L

Har. 1433

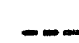

HMM- $6 \times 9, M$

Colo.
USGS-XS

USNM-9x9, L 
Gilpinite---Specimen Data (Cont.)

\begin{tabular}{|c|c|c|c|}
\hline $\begin{array}{l}\text { Mineral and identi- } \\
\text { fying number }\end{array}$ & Location & $\begin{array}{l}\text { Ref. no. of } \\
\text { X-ray patterns }\end{array}$ & Quantity \\
\hline $\begin{array}{l}\text { Gilpinite } 2 / \\
\text { USNM } 49090\end{array}$ & $\begin{array}{l}\text { Central City, } \\
\text { Colo. }\end{array}$ & -- & -- \\
\hline
\end{tabular}

1 Larsen, E. S. and Berman, Harry, Am. Mineralogist 11, I (1926). 2) Larsen, E. S., and Brown, G. V., Am. Mineralogist 2, 75 (1917).

KASOLITE $\mathrm{Pb}\left(\mathrm{UO}_{2}\right) \mathrm{S}_{4} \mathrm{O}_{4} \cdot \mathrm{H}_{2} \mathrm{O}$

Specimen Data

\begin{tabular}{|c|c|c|c|}
\hline $\begin{array}{l}\text { Mineral and identi- } \\
\text { fying number }\end{array}$ & Location & $\begin{array}{l}\text { Ref. no. of } \\
\text { X-ray patterns }\end{array}$ & Quantity \\
\hline $\begin{array}{l}\text { Kasolite } \\
\text { USNM } 95610\end{array}$ & Belgian Congo & TE 634 & $\begin{array}{l}\text { USGS-XS } \\
\text { USNM-20 g }\end{array}$ \\
\hline $\begin{array}{l}\text { Kasolite } \\
\text { USNM C4517 }\end{array}$ & $\begin{array}{l}\text { Chinkolobwe, } \\
\text { Katanga, Belgian Congo }\end{array}$ & -- & $\begin{array}{l}\text { USGS-XS } \\
\text { USNM-20 } g\end{array}$ \\
\hline $\begin{array}{l}\text { Kasolite } \\
\text { HMM } 86781 \\
\text { Phase } 79\end{array}$ & $\begin{array}{l}\text { Katanga, } \\
\text { Belgian Congo }\end{array}$ & Har. unnumbered &.-- \\
\hline
\end{tabular}

LIEBIGITE $\mathrm{Ca}_{2} \mathrm{U}\left(\mathrm{CO}_{3}\right)_{4} \cdot 10 \mathrm{H}_{2} \mathrm{O}$

Optics I/
Orientation $n$
$X \quad a$
1.497
$Y$
1.502
1.539
Pleochrolsm
Nearly colorless
Pale yellowish green
Biaxial positive $(t)$
Pale yellowish green
$2 \mathrm{~V}=40^{\circ}$
$r>v$, moderate
Specimen Data

$\begin{array}{llll}\begin{array}{l}\text { Mineral and identi- } \\ \text { fying number }\end{array} & \text { Location } & \begin{array}{l}\text { Ref. no. of } \\ \text { X-ray patterns }\end{array} & \text { Quantity } \\ \begin{array}{l}\text { Liebigite } \\ \text { HMM Phase } 18\end{array} & \begin{array}{l}\text { Joachimsthal, } \\ \text { Bohemia }\end{array} & \text { Har. } 1767 & \ldots\end{array}$


Liebigite---Specimen Data (Cont.)

\begin{tabular}{|c|c|c|c|}
\hline $\begin{array}{l}\text { Mineral and identi- } \\
\text { fying number }\end{array}$ & Location & $\begin{array}{l}\text { Ref. no. of } \\
X \text {-ray patterns }\end{array}$ & Quantity \\
\hline $\begin{array}{l}\text { Liebigite } \\
\text { USNM R6753 }\end{array}$ & $\begin{array}{l}\text { Joachimsthal, } \\
\text { Bohemia }\end{array}$ & TE 594 & $\begin{array}{l}\text { USGS-XS } \\
\text { USNM-9x } 12, I\end{array}$ \\
\hline $\begin{array}{l}\text { Liebigite } \\
\text { AMNH } 8976\end{array}$ & $\begin{array}{l}\text { Joa chimsthal, } \\
\text { Bohemia }\end{array}$ & -- & HMM-9×9, I \\
\hline
\end{tabular}

Uranothallite--Synonym of Iiebigite

Uranothallite

HMM 93993

Uranothallite

USNM R276I

Uranothallite

TE (CFM-I)
Joachimsthal,

Bohemia

Joachimsthal,

Bohemia
HMM- $3 \times 3, L$

USGS-XS

USNM-3x9, L

If Frondel, Clifford, Harvard University, personal communication.

$$
\text { MACKINTOSHITE }(\mathrm{Th}, \mathrm{U}) \mathrm{SiO}_{4} \cdot \mathrm{H}_{2} \mathrm{O} \text { ? }
$$

Specimen Data

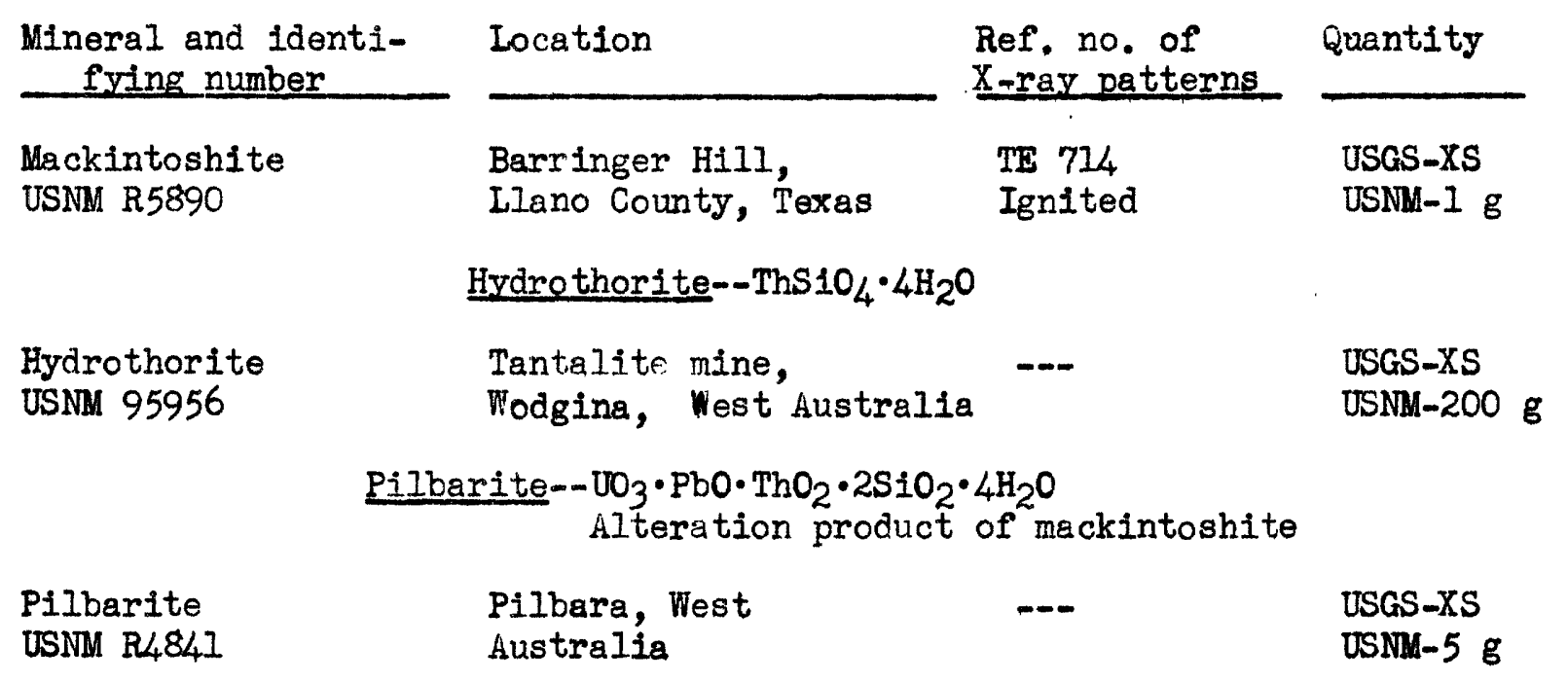


MAITLANDITE $(\mathrm{U}, \mathrm{Th}, \mathrm{Pb}) \mathrm{SiO}_{4} \cdot 3 \mathrm{H}_{2} \mathrm{O}$ ?

Specimen Data

\begin{tabular}{|c|c|c|c|}
\hline $\begin{array}{l}\text { Mineral and ident1- } \\
\text { fying number }\end{array}$ & Location & $\begin{array}{l}\text { Ref. no. of } \\
\text { x-ray patterns }\end{array}$ & Quantity \\
\hline $\begin{array}{l}\text { Maitlandite } \\
\text { USMM } 95958\end{array}$ & $\begin{array}{l}\text { Wodgina, West } \\
\text { Australia }\end{array}$ & $\begin{array}{l}\text { TE } 586 \\
\text { TE } 722 \\
\text { Ignited }\end{array}$ & $\begin{array}{l}\text { USGS-XS } \\
\text { USNM-I }\end{array}$ \\
\hline
\end{tabular}

Nicolayite--Close to maitlandite

Nicolayite

Wodgina, West

USGS-X

USNM 95959

Australia

USNM-5 $\mathrm{g}$

MEDJIDITE--Sulfate of uranium and calcium

Specimen Data

\begin{tabular}{|c|c|c|c|}
\hline $\begin{array}{l}\text { Mineral and identi- } \\
\text { fying number }\end{array}$ & Location & $\begin{array}{l}\text { Ref. no. of } \\
\text { X-ray patterns }\end{array}$ & Quantity \\
\hline $\begin{array}{l}\text { Medjidite } \\
\text { USNM R6331 }\end{array}$ & $\begin{array}{l}\text { Joachimsthal, } \\
\text { Bohemia }\end{array}$ & --- & $\begin{array}{l}\text { USGS-XS } \\
\text { USNM-2O g }\end{array}$ \\
\hline $\begin{array}{l}\text { Medjidite } \\
\text { AMNH } 16836\end{array}$ & $\begin{array}{l}\text { Joachimsthal, } \\
\text { Bohemia }\end{array}$ & -- & HMM $-3 \times 9, L$ \\
\hline
\end{tabular}

$$
\begin{gathered}
\text { METATORBERNITE } \mathrm{Cu}\left(\mathrm{UO}_{2}\right)_{2}\left(\mathrm{PO}_{4}\right)_{2} \cdot 8 \mathrm{H}_{2} \mathrm{O} \\
\text { Optics }
\end{gathered}
$$

Optically uniaxial; some is biaxial due to strain, $2 \mathrm{~V}$ small. Pleochroic, 0 - sky blue, E = green.

$\begin{array}{cclcc} & 640 \mathrm{~m} \mu & 515 \mathrm{~m} \mu & 440 \mathrm{~m} \mu & \text { white light } \\ \text { no } & 1.618 & 1.633 & 1.649 & 1.610-1.628 \\ \mathrm{nE} & 1.622 & & 1.646 & (+) \\ \text { Sign } & (+) & \text { isotropic } & (-) & \end{array}$


Metatorbernite (Cont.)

\section{Specimen Data}

\begin{tabular}{|c|c|c|c|}
\hline $\begin{array}{l}\text { Mineral and identi- } \\
\text { fying number }\end{array}$ & Iocation & $\begin{array}{l}\text { Ref. no. of } \\
\text { X-ray patterns }\end{array}$ & Quantity \\
\hline $\begin{array}{l}\text { Metatorbernite } \\
\text { USNM R5682 }\end{array}$ & $\begin{array}{l}\text { Old Gunnis Lake mi } \\
\text { Calstock, Cornwall }\end{array}$ & Land & $\begin{array}{l}\text { USGS-XS } \\
\text { USNM-10 g }\end{array}$ \\
\hline $\begin{array}{l}\text { Metatorbernite } \\
T E\end{array}$ & $\begin{array}{l}\text { Mina de Urgeiriça, } \\
\text { Portugal }\end{array}$ & TE 435 & USGS-XSC \\
\hline
\end{tabular}

$$
\begin{gathered}
\text { METAZEUNERITE } \mathrm{Cu}\left(\mathrm{OO}_{2}\right)_{2}\left(\mathrm{AsO}_{4}\right)_{2} \cdot 8 \mathrm{H}_{2} \mathrm{O} \\
\text { Optics }
\end{gathered}
$$

In transmitted Iight, green. Weakly dichroic.

$\begin{array}{ccccl} & \mathrm{n} \text { (Schneeberg) I/ } & \mathrm{n}(\text { Tintic) } 2 / & \mathrm{n} \text { (Schneeberg) } 1 / & \text { Dichroism } \\ 0 & 1.643 & 1.647 & 1.651 & \text { Grass green } \\ \mathrm{E} & 1.623 & 1.630 & 1.635 & \text { Pale green }\end{array}$

Specimen Data

\begin{tabular}{|c|c|c|c|}
\hline $\begin{array}{l}\text { Orientation } \\
\underset{X}{Y} \mathrm{~b} \\
\underset{\mathrm{Z}}{A_{C}}=2{ }^{\circ}=2^{\circ} 6^{\circ}\end{array}$ & $\begin{array}{c}\mathrm{n}(\mathrm{Na}) \text { Brazil I/ } \\
1.7902 \\
1.7912 \\
1.8441 \\
15^{\circ} 331\end{array}$ & $\begin{array}{c}\mathrm{n}(\mathrm{Na}) \text { Madagascar } 2 / \\
1.8004 \\
1.8008 \\
1.8494 \\
110001\end{array}$ & $\begin{array}{l}\text { n Bolivia } 3 \\
1.785 \\
1.787 \\
1.840\end{array}$ \\
\hline
\end{tabular}

$$
\begin{aligned}
& \text { Mineral and identi- Location } \\
& \text { fring number } \\
& 6 \times 6,11 \\
& \text { MONAZITE (Ce, La, } \mathrm{Y}, \mathrm{Th}) \mathrm{PO}_{4} \\
& \text { Optics }
\end{aligned}
$$

In transmitted light, yellowish brown or yellow to colorless.

$r<v$, in some $r>v$; horizontal dispersion weak. Pleochroism faint or not perceptible. 
Monazite (Cont.)

Specimen Data

\begin{tabular}{|c|c|c|c|}
\hline $\begin{array}{l}\text { Mineral and identi- } \\
\text { fying number }\end{array}$ & Location & $\begin{array}{l}\text { Ref. no. of } \\
\text { X-ray patterns }\end{array}$ & Quantity \\
\hline $\begin{array}{l}\text { Monazite } \\
\text { USNM R4339 }\end{array}$ & $\begin{array}{l}\text { Spruce Pine, } \\
\text { N.C. }\end{array}$ & -- & $\begin{array}{l}\text { USGS-XS } \\
\text { USNM-100 g }\end{array}$ \\
\hline $\begin{array}{l}\text { Monazite } 4 / \\
\text { USNM } 102846\end{array}$ & $\begin{array}{l}\text { Mars Hill, } \\
\text { N.C. }\end{array}$ & -- & USNM-25g \\
\hline $\begin{array}{l}\text { Monazite } 5 / \\
\text { USNM 100282-86 }\end{array}$ & $\begin{array}{l}\text { Morefield mine, } \\
\text { Amelia, } \mathrm{Va} \text {. }\end{array}$ & --- & USNM-75 g \\
\hline $\begin{array}{l}\text { Monazite } \\
\mathrm{TE}(517-7-\mathrm{B})\end{array}$ & $-\infty-$ & TE 248 & --- \\
\hline $\begin{array}{l}\text { Monazite } \\
\text { USNM } 105008\end{array}$ & $\begin{array}{l}\text { Luwangashi, } \\
\text { Belgian Congo }\end{array}$ & -- & USGS-XS \\
\hline $\begin{array}{l}\text { Monazite } 6 / \\
\text { USNM } 96870\end{array}$ & $\begin{array}{l}\text { Divino de Ubá, } \\
\text { Minas Geraes, Brazil }\end{array}$ & -- & USNM-200 g \\
\hline $\begin{array}{l}\text { Monazite } \\
\text { HMM }\end{array}$ & Arendal, Sweden & Har. 5079 & -- \\
\hline
\end{tabular}

Menglte--Symonym of monazite

Mengite

USNM R5099

Turnerite

USNM C4033

Urdite

USNM R5168
Ilmen Mountains, Urals, Russia
USGS-XS

USMM-10 g

Turnerite--Synonym of monazite

Binnenthal,

Switzerland

$\ldots$

USGS-X

USNM-20 g

Urdite--Synonym of monazite
USGS-XS

USNM-50 g

If Busz, Neues Jahrb., Beilage-Band 39, 492 (1914).

2/ Sabot, R. C., Inaug. Diss. Ghent (1914); Neues Jahrb. 138 (1920).

3/ Gordon, S. G., Acad. Nat. Sc1. Philadelphia, Not. nat. 2 (1939).

4 Marble, J. P., Am. Mineralogist 21, 456-57 (1936).

5/ Glass, J. J., Am. Mineralogist 20,741-68 (1935).

6) Fenner, C. N., Am. Jour. Sci., 5th ser., 16, 382-91 (1928). 
PARASC HOEPITE $\mathrm{JO}_{3} \cdot 2 \mathrm{H}_{2} \mathrm{O}$ ?

Optics I/

$\begin{array}{ccc}\text { Orientation } & \mathrm{n} \\ \mathrm{X} & \mathrm{c} & 1.705 \\ \mathrm{Y} & \mathrm{b} & 1.760 \\ \mathrm{Z} & \mathrm{a} & 1.770\end{array}$
Pleochroism

Colorless or nearly so Eiaxial negative (-)

Yellow $2 \mathrm{~V} \sim 40^{\circ}$

$r<v$

\title{
Specimen Data
}

\author{
Mineral and identi- Location \\ fying number \\ Paraschoepite \\ $\operatorname{HMM}(\mathrm{K}-\mathrm{I})$ \\ Katanga, \\ Belgian Congo \\ Ref. no. of Quantity \\ Paraschoepite \\ HMM Phase 99 \\ Type specimen \\ Katanga, \\ Belgian Congo \\ X-ray patterns \\ TE 66 \\ HMM- $1 / 5 \mathrm{~g}$ \\ Har. unnumbered HMM- $1 / 5 \mathrm{~g}$
} If Schoep, Alfred, and Stradiot, Sadi, Am. Mineralogist 32, 344-350

$$
\begin{gathered}
\text { PARSONSITE } \mathrm{Pb}_{2}\left(\mathrm{UO}_{2}\right)\left(\mathrm{PO}_{4}\right)_{2} \cdot 2 \mathrm{H}_{2} \mathrm{O} \\
\text { Optics }
\end{gathered}
$$

\begin{tabular}{|c|c|c|c|}
\hline Orientation & $\begin{array}{c}\mathrm{n}(\text { Kasola }) I / \\
1.85\end{array}$ & $\begin{array}{c}\text { n(Ruggles) } \\
\sim 1.870\end{array}$ & $\begin{array}{c}n(\text { Wölsendorf }) \\
\sim 1.795\end{array}$ \\
\hline $\begin{array}{l}\mathrm{Y} \quad \mathrm{b} \\
\mathrm{Z} \\
\mathrm{Z} \wedge \mathrm{c} \text { (usually) }\end{array}$ & $\begin{array}{r}1.852 \\
12^{\circ}\end{array}$ & $\tau^{1} .890$ & $\sim^{7} \cdot 815$ \\
\hline
\end{tabular}

In transmitted light, pale yellow. Not pleochroic. Biaxial negative(-

\begin{tabular}{|c|c|c|c|}
\hline $\begin{array}{l}\text { Mineral and identi- } \\
\text { fying number }\end{array}$ & Location & $\begin{array}{l}\text { Ref. no. of } \\
\text { X-ray patterns }\end{array}$ & Quantity \\
\hline $\begin{array}{l}\text { Parsonsite } \\
\text { USNM } 97139\end{array}$ & $\begin{array}{l}\text { Katanga, } \\
\text { Belgian Congo }\end{array}$ & $\mathrm{TE} 3141$ & USNM-20 g \\
\hline $\begin{array}{l}\text { Parsonsite } \\
\text { USNM } 95605\end{array}$ & $\begin{array}{l}\text { Chinkolobwe, } \\
\text { Belgian Congo }\end{array}$ & -- & $\begin{array}{l}\text { USGS-XS } \\
\text { USNM-10 g }\end{array}$ \\
\hline
\end{tabular}

Specimen Data

If Billiet, V., Soc. frang. minéralogie Bull. 49, 136 (1926). 
PHOSPHURANYLITE--A lead, calcium, uranium phosphate

\section{Optics}

Some is uniaxial negative $(-)$, but ordinarily it is biaxial negative $(-)$. $2 \mathrm{~V}$ variable and ranges up to about $35^{\circ}$ but usually is $5^{\circ}$ to $20^{\circ}$.

Orientation $n$ (Flat Rock $n$ (Ruggles, $n$ (Rosmaneira, Pleochrolsm $\begin{array}{lcccc} & \text { mine, N.C.) } & \text { mine, N.H.) } & \text { Portugal) } & \\ \mathrm{X} \text { or } \mathrm{E} & 1.690 & 1.668 & 1.660 & \text { Colorless to pale yellow } \\ \mathrm{Y} \text { or } 0 & 1.718 & 1.710 & 1.700 & \text { Golden yellow } \\ \mathrm{Z} \text { or } & 1.718 & 1.710 & 1.701 & \text { Golden yellow }\end{array}$

In blaxial material $r>v$, strong.

Specimen Data

\begin{tabular}{|c|c|c|c|}
\hline $\begin{array}{l}\text { Mineral and identi- } \\
\text { fying number }\end{array}$ & Location & $\begin{array}{l}\text { Ref. no. of } \\
\text { X-ray patterns }\end{array}$ & Quantity \\
\hline $\begin{array}{l}\text { Phosphuranylite } \\
\text { USNM } 104824\end{array}$ & $\begin{array}{l}\text { Rio Grande do } \\
\text { Norte, Brazil }\end{array}$ & $\mathrm{TE} 3127$ & $\begin{array}{l}\text { USGS-XS } \\
\text { USNM-100g }\end{array}$ \\
\hline $\begin{array}{l}\text { Phosphuranylite } \\
\text { HMM }\end{array}$ & $\begin{array}{l}\text { Rosmaneira, } \\
\text { Portugal }\end{array}$ &.-- & HMM- $5 \mathrm{~g}$ \\
\hline
\end{tabular}

$$
\begin{gathered}
\text { POLYCRASE }(\mathrm{Y}, \mathrm{Ca}, \mathrm{Ce}, \mathrm{U}, \mathrm{Th})(\mathrm{TI}, \mathrm{Cb}, \mathrm{Ta})_{2} \mathrm{O}_{6} \\
\text { Optics }
\end{gathered}
$$

In transmitted light, brown, yellow brown, or reddish brown. Isotropic (metamict). The refractive index increases after ignition.

\begin{tabular}{|c|c|c|c|}
\hline $\begin{array}{l}\text { Mineral and identi- } \\
\text { fying number }\end{array}$ & Location & $\begin{array}{l}\text { Ref. no. of } \\
\text { X-ray patterns }\end{array}$ & Quantity \\
\hline $\begin{array}{l}\text { Polycrase } \\
\text { TE }\end{array}$ & $\begin{array}{l}\text { Overlook, } \\
\text { N.Y. }\end{array}$ & $\begin{array}{l}\text { TE } 478 \\
\text { Ignited }\end{array}$ & USGS-XSC \\
\hline $\begin{array}{l}\text { Polycrase } \\
\text { USNM } 97642\end{array}$ & $\begin{array}{l}\text { San Jose de Bujauba, } \\
\text { Minas Geraes, Brazil }\end{array}$ & $\begin{array}{l}\text { TE } 749 \\
\text { Ignited }\end{array}$ & $\begin{array}{l}\text { USGS-XS } \\
\text { USNM-100 }\end{array}$ \\
\hline
\end{tabular}

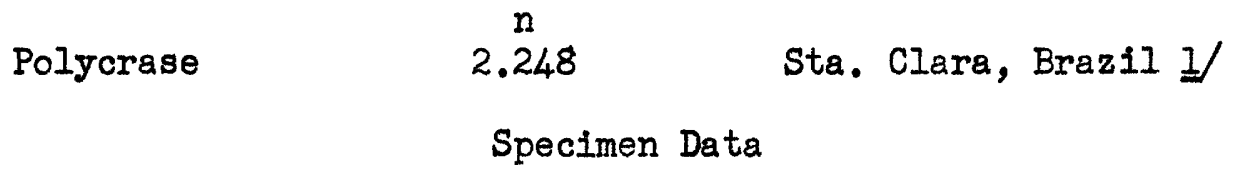


Polycrase----Specimen Data (Cont.)

\begin{tabular}{|c|c|c|c|}
\hline $\begin{array}{l}\text { Mineral and identi- } \\
\text { fying number }\end{array}$ & Location & $\begin{array}{l}\text { Ref. no. of } \\
\text { X-ray patterns }\end{array}$ & Quantity \\
\hline $\begin{array}{l}\text { Polycrase } \\
\text { HMM } 87869\end{array}$ & $\begin{array}{l}\text { Hitter"̈, } \\
\text { Norway }\end{array}$ & $\begin{array}{l}\text { Har. } 1712 \\
\text { Ignited } \\
3 \mathrm{hrs} \text {. to } \\
\text { red heat }\end{array}$ & $--\infty$ \\
\hline
\end{tabular}

I/ Hess, F. L., and Henderson, E. P., Franklin Inst. Jour. 200, 235 (1925).

$$
\begin{gathered}
\text { POLDIGNYTT } \mathrm{A}(\mathrm{Cb}, \mathrm{Ti}, \mathrm{Ta}) \mathrm{O}_{4} \\
\mathrm{~A}=\mathrm{Ca}, \mathrm{Fe}^{2}, \mathrm{Y}, \mathrm{etc}, \mathrm{Zr}, \mathrm{Th}^{4} \\
\text { Optics }
\end{gathered}
$$

In transmitted light, reddish brown and isotropic (metamict). $\mathrm{n}=2.22 \pm 0.01 \mathrm{I} /$ (Fredricksvärn).

\section{Specimen Data}

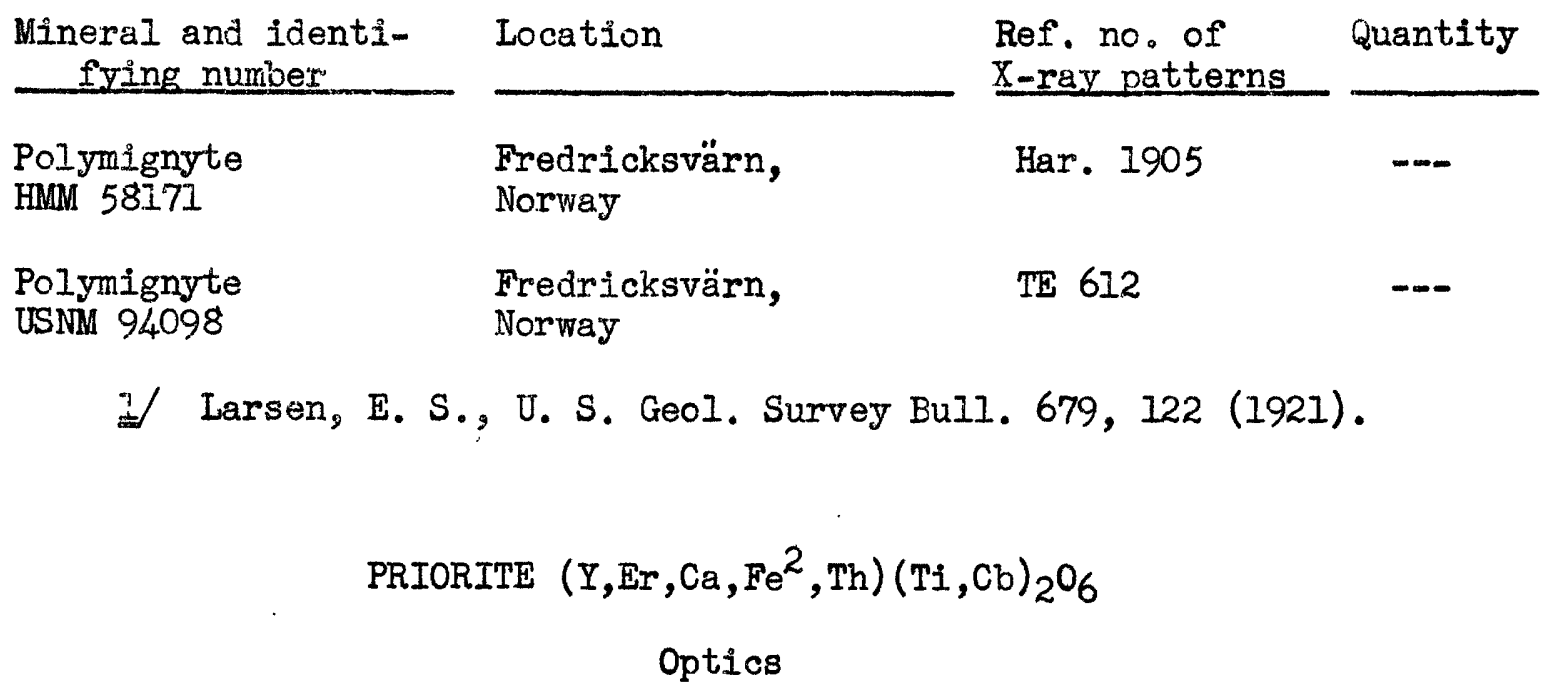

Isotropic (metamict) and transmits light in only the thinnest splinters Reddish brown to light brown. Becomes anisotroplc on lgnitiof, and the refractive index increases somewhat. 1 / Priorite $n=2.142 \pm 0.01$, after ignition $n=2.24$. 
Priorite (Cont.)

Specimen Data

\begin{tabular}{|c|c|c|c|}
\hline $\begin{array}{l}\text { Mineral and identi- } \\
\text { fying number }\end{array}$ & Location & $\begin{array}{l}\text { Ref. no. of } \\
X \text {-ray patterns }\end{array}$ & Quantity \\
\hline $\begin{array}{l}\text { Priorite } \\
\text { HMM } 85140\end{array}$ & $\begin{array}{l}\text { Ahi-Tromby, } \\
\text { Madagascar }\end{array}$ & $\begin{array}{l}\text { Har. } 1837 \\
\text { Ignited }\end{array}$ & $\mathrm{BMM}-75 \mathrm{~B}$ \\
\hline $\begin{array}{l}\text { Priorite } \\
\text { HMM } 80039\end{array}$ & $\begin{array}{l}\text { Iveland, Satersdalen, } \\
\text { Norway }\end{array}$ & $\begin{array}{l}\text { Har. } 1880 \\
\text { Ignited }\end{array}$ & $\cdots$ \\
\hline $\begin{array}{l}\text { Priorite } \\
\text { HMM }\end{array}$ & --- & Har. 1837 & $-\cdots$ \\
\hline$\underline{\mathrm{B} I 0}$ & trandine--Synonym of $\mathrm{pr}$ & rite & \\
\hline $\begin{array}{l}\text { Blomstrandine } \\
\text { USNM C4019 }\end{array}$ & $\begin{array}{l}\text { Iveland, Satersdalen, } \\
\text { Norway }\end{array}$ & $\begin{array}{l}\text { TE } 756 \\
\text { Ignited }\end{array}$ & $\begin{array}{l}\text { USGS-XS } \\
\text { USNM-100 g }\end{array}$ \\
\hline $\begin{array}{l}\text { Blomstrandine } \\
\text { HMM } 84647\end{array}$ & $\begin{array}{l}\text { Morefjaer, Arendal, } \\
\text { Norway }\end{array}$ & $\begin{array}{l}\text { Har. } 1871 \\
\text { Ignited }\end{array}$ & $\mathrm{HMM}-100 \mathrm{~g}$ \\
\hline $\begin{array}{l}\text { Blomstrandine } \\
\text { HMM } 87873\end{array}$ & $\begin{array}{l}\text { Tongafeno, } \\
\text { Madagascar }\end{array}$ & $\begin{array}{l}\text { Har. } 2028 \\
\text { Inv. } 2784\end{array}$ & $\mathrm{HMM}-50 \mathrm{~g}$ \\
\hline
\end{tabular}
I/ Larsen, E. S., and Berman, Harry, U. S. Geol. Survey Bull. 848,
65 (1934).

$$
\begin{aligned}
& \text { RAWITE } \mathrm{CaU}_{2} \mathrm{~V}_{12} \mathrm{O}_{36} \cdot 2 \mathrm{OH}_{2} \mathrm{O} \\
& \text { Optics }
\end{aligned}
$$

Mean index of refraction 1.88 .

\begin{tabular}{|c|c|c|c|}
\hline Mineral and identi- & Location & $\begin{array}{l}\text { Ref. no. of } \\
X \text {-ray patterns }\end{array}$ & Quantity \\
\hline $\begin{array}{l}\text { Rauvite } \\
\text { USNM } 96159\end{array}$ & $\begin{array}{l}\text { Yellow Cat district, } \\
\text { Grand County, Utah }\end{array}$ & --- & $\begin{array}{l}\text { USGS-XS } \\
\text { USNM-100 g }\end{array}$ \\
\hline $\begin{array}{l}\text { Rauvite } \\
\text { USNM } 96054\end{array}$ & $\begin{array}{l}\text { Yellow Cat Wash, } \\
\text { Grand County, Utah }\end{array}$ & -- & $\begin{array}{l}\text { USAS-XS } \\
\text { USNM-300 g }\end{array}$ \\
\hline
\end{tabular}

Specimen Data 
Rauvite---Specimen Data (Cont.)

\begin{tabular}{|c|c|c|c|}
\hline $\begin{array}{l}\text { Mineral and identi- } \\
\text { fying number }\end{array}$ & Location & $\begin{array}{l}\text { Ref. no. of } \\
\text { X-ray patterns }\end{array}$ & Quantity \\
\hline $\begin{array}{l}\text { Rauvite } \\
\text { USNM C4417 }\end{array}$ & $\begin{array}{l}\text { Temple Mountain } \\
\text { (San Rafael Swell) } \\
\text { Emery County, Utah }\end{array}$ & TE 3135 & $\begin{array}{l}\text { USGS-XS } \\
\text { USNM-5 } g\end{array}$ \\
\hline $\begin{array}{l}\text { Rauvite } 1 / 1 \\
\text { USNM } 95060\end{array}$ & $\begin{array}{l}\text { Temple Mountain } \\
\text { (San Rafael Swell) } \\
\text { Emery County, Utah }\end{array}$ & $\begin{array}{l}\mathrm{TE} 3167 \\
\text { Har. } 422\end{array}$ & $\begin{array}{l}\text { USNM-100 g } \\
\text { HMM- } 1 \mathrm{~g}\end{array}$ \\
\hline
\end{tabular}

$$
\text { RENARDITE } \mathrm{Pb}\left(\mathrm{UO}_{2}\right)_{4}\left(\mathrm{PO}_{4}\right)_{2}(\mathrm{OH})_{4} \cdot 7 \mathrm{H}_{2} \mathrm{O}
$$

$$
\text { Optics }
$$

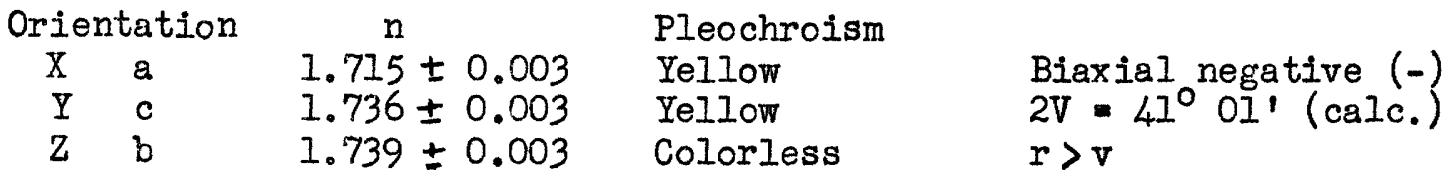

$$
\begin{aligned}
& \text { Specimen Data } \\
& \text { RUTHERFORDINE } \mathrm{UO}_{2} \mathrm{CO}_{3} \text { ? } \\
& \text { Optics I/ }
\end{aligned}
$$

In transmitted light, yellow. Optically biaxial with: $n X 1.72 \pm 0.01$ $\mathrm{nZ} 1.80 \pm 0.01$

If Larsen, E. S., U. S. Geol. Survey Bull. 679, 129 (1921). 


$$
\text { SALÉEITE } \mathrm{Mg}\left(\mathrm{UO}_{2}\right)_{2}\left(\mathrm{PO}_{4}\right)_{2} \cdot 1 \mathrm{OH}_{2} \mathrm{O}
$$

Optics

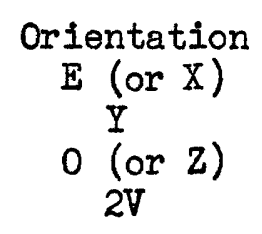

$$
\begin{array}{cc}
n(\text { Schneeberg) } 1 / & n(\text { Katanga }) 2 / \\
1.559 \pm 0.002 \text { Colorless } & 1.559 \\
& 1.570 \\
1.574 \pm 0.002 \text { Pale greenish yellow } & 1.574 \\
& r>v, \text { strong }
\end{array}
$$

Uniaxial negative (-), some is anomalously biaxial.

Specimen Data
Mineral and identi-
Location
Ref. no. of
Quantity
fying number

\section{USNM R8385}
Saléeite
Saléeite
HMM

\section{X-ray patterns}
TE 655
USGS-XS
USNM-4x9, I
HMM- $3 \times 9$, I
If Mrose, M. E., Am. Mineralogist 35, 525-530 (1950).
Chinkolobwe, Katanga,
Belgian Congo
Chinkolobwe, Katanga,
Belgian Congo
2) Schoep, Alfred., Med. Kl. Wetens. Kon. Vlaam. Acad., 65 (1939).
SAMARSKITE ( $\mathrm{Y}, \mathrm{Er}, \mathrm{U}, \mathrm{Ca}, \mathrm{Fe}, \mathrm{Th})(\mathrm{Cb}, \mathrm{Ta})_{2} \mathrm{O}_{6}$

Optics

In transmitted light, light brown to dark brown. Ordinarily metamict and isotropic. Anisotropic samarskite has been reported but without verification. I/ Refractive index variable, with $\mathrm{n}=$ $2.20 \pm 0.05$ reported on unanalyzed material. $2 /$

Specimen Data

Mineral and identi- Location

fying number

Samarskite $3 /$

USNM 49088

Samarskite

HMM 92576
Devil's Head Mountain near Pikes Peak, Colo.

Spinelli quarry, Glastonbury, Conn.
Ref. no. of X-ray patterns

USNM-40 g

Quantity

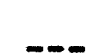

Har. 1879

Ignited 
Samarskite---Specimen Data (Cont.)

\begin{tabular}{|c|c|c|c|}
\hline $\begin{array}{l}\text { Mineral and identi- } \\
\text { fying number }\end{array}$ & Location & $\begin{array}{l}\text { Ref. no. of } \\
X \text {-ray patterns }\end{array}$ & Quantity \\
\hline $\begin{array}{l}\text { Samarskite } \\
\text { HMM } 81133\end{array}$ & $\begin{array}{l}\text { Wiseman's mine, } \\
\text { Mitchell County, N.C. }\end{array}$ & $\begin{array}{l}\text { Har. } 1900 \\
\text { Ignited }\end{array}$ & -- \\
\hline $\begin{array}{l}\text { Samarskite } \\
\text { HMM } 58012\end{array}$ & $\begin{array}{l}\text { Mitchell County } \\
\text { N.C. }\end{array}$ & $\begin{array}{l}\text { Har. } 1916 \\
\text { Ignited }\end{array}$ & --- \\
\hline $\begin{array}{l}\text { Samarskite } \\
\text { HMM } 58011\end{array}$ & $\begin{array}{l}\text { Yancey County } \\
\text { N.C. }\end{array}$ & $\begin{array}{l}\text { Har. } 1891 \\
\text { Ignited }\end{array}$ & $-\infty$ \\
\hline $\begin{array}{l}\text { Samarskite } \\
\text { HMM } 92510\end{array}$ & $\begin{array}{l}\text { Topsham, } \\
\text { Maine }\end{array}$ & $\begin{array}{l}\text { Har. } 1889 \\
\text { Ignited }\end{array}$ & $\mathrm{HN}-10 \mathrm{~g}$ \\
\hline $\begin{array}{l}\text { Samarskite } 4 / \\
\text { USNM } 96115\end{array}$ & $\begin{array}{l}\text { Petaca, } \\
\text { N. Mex. }\end{array}$ & --- & $\begin{array}{l}\text { USGS-XS } \\
\text { USNM-175g }\end{array}$ \\
\hline Samarskite & $\begin{array}{l}\text { Mitchell County, } \\
\text { N.C. }\end{array}$ & $\begin{array}{l}\text { Inv. } 4469 \\
\text { Ignited }\end{array}$ & $\therefore$ \\
\hline Samarskite 5/ & $\begin{array}{l}\text { Mitchell County, } \\
\text { N.C. }\end{array}$ & $\begin{array}{l}\text { Inv. } 4474 \\
\text { Ignited }\end{array}$ & -- \\
\hline $\begin{array}{l}\text { Samarskite } \\
\text { USNM } 96872\end{array}$ & $\begin{array}{l}\text { Divino de Ubá, } \\
\text { Minas Geraes, Brazil }\end{array}$ & -- & $\begin{array}{l}\text { USGS-XS } \\
\text { USNM-200 g }\end{array}$ \\
\hline $\begin{array}{l}\text { Samarskite } \\
\mathrm{TE}(404 \text { AT0-2) }\end{array}$ & -- & $\begin{array}{l}\text { TE } 169 \\
\text { Ignited }\end{array}$ & -- \\
\hline Samarskite & $\ldots$ & --- & USGS-XSC \\
\hline
\end{tabular}

Annerödite--Mixture of samarskite and parallel growths of columbite

Annerödite

USNM R7142

$$
\begin{aligned}
& \text { Anneröd, Moss, } \\
& \text { Norway }
\end{aligned}
$$

USGS-XS

USNM-75 g

\section{Vietinghofite--Synonym of samarskite}

Viet,inghofite

USNM R5II8
Baikal Lake,

Siberia
USGS-XS

USNM-20 $\mathrm{g}$

If Van Aubel, René, Soc. géol. Belgique Annales 58, Pub. vel. Congo belge, Fasc. 38 (1935) -.. Neues Jahrb. Mineralogie I (1945), on material from Kivu, Belgian Congo.

2/ Larsen, E. S., U. S. Geol. Survey Bul1. 679 129, 158 (1921).

3/ Hillebrand, W. F., U. S. Geol. Survey Bul1. 55, (1889).

4 Hess, F. L., and Wells, R. C., Am. Jour. Sci., Sth ser., 19, $17-26(1930)$.

5/ Davidson, Norman, U. S. Geol. Survey, Analysis D-1763. 
SCHOEPITE $4 \mathrm{UO}_{3} \cdot 9 \mathrm{H}_{2} \mathrm{O}$

Optics

Yellow in transmitted light.

Orientation

$\begin{array}{ll}\mathrm{X} & \mathrm{c} \\ \mathrm{Y} & \mathrm{b} \\ \mathrm{Z} & \mathrm{a}\end{array}$
Pleochroism

Colorless

Lemon yellow

Lemon yellow
Biaxial negative (-) $2 \mathrm{~V} 89^{\circ}$

$r>v$

Specimen Data

Ref. no. of Quantity X-ray patterns TE 615

USGS-X USNM-30 $\mathrm{g}$

USGS-XS USNM-20 $\mathrm{g}$

HMMM- $3 \times 3, \mathrm{~L}$

Har. 1571

Belgian Congo

Great Bear Lake, Canada

$\operatorname{taM}-6 \times 6, \mathrm{~L}$

Phase 59

I) Walker, T. I., Am. Mineralogist 8, 67 (1923).

SCHROECKINGERITE $\mathrm{Ca}_{3} \mathrm{NaUO}_{2}\left(\mathrm{CO}_{3}\right)_{3}\left(\mathrm{SO}_{4}\right) \mathrm{F} \cdot 1 \mathrm{OH}_{2} \mathrm{O}$

Optics

Usually biaxial with small and variable $2 \mathrm{~V}, 0^{\circ}-25^{\circ}$.

$\begin{array}{cccc} & \mathrm{n} \text { (Wyoming) } 1 / & \mathrm{n} \text { (Joachimsthal)2/ } & \text { Oniaxial ? } \\ 0 & 1.542 & 1.539-1.545 & \text { negative (-) } \\ \mathrm{E} & 1.489 & 1.496 & \end{array}$

Specimen Data

Mineral and identi- Location fying number

Schroeckingerite
Ref. no. of $X$-ray patterns

Yavapai County, Ariz.
Inv. 2765 
Schroeckingerite---Specimen Data (Cont.)

\begin{tabular}{|c|c|c|c|}
\hline $\begin{array}{l}\text { Mineral and identi- } \\
\text { fying number }\end{array}$ & Location & $\begin{array}{l}\text { Ref. no. of } \\
\text { X-ray patterns }\end{array}$ & Quantity \\
\hline Schroeckingerite & Near Wamsutter, Wyo. & -- & USGS-XSC \\
\hline $\begin{array}{l}\text { Schroeckingerite } \\
\text { HMM } 92877\end{array}$ & Near Wamsutter, Wyo. & --- & $\mathrm{HM}-20 \mathrm{~g}$ \\
\hline $\begin{array}{l}\text { Schroeckingerite } \\
\text { HMM Phase } 5\end{array}$ & $\ldots$ & Har. 1591 & $-\infty$ \\
\hline $\begin{array}{l}\text { Schroeckingerite } \\
\text { AMNH } 8979\end{array}$ & $\begin{array}{l}\text { Joachimsthal, } \\
\text { Bohemia }\end{array}$ & -- & $\mathrm{HMM}$ \\
\hline
\end{tabular}

\section{Dakeite--Synonym of schroeckingerite}

Dakeite 3/

Near Wamsutter, Wyo. TE 609

USGS-XS

USNM 103010

USNM-20 $\mathrm{g}$

I/ Larsen, E. S., and Gonyer, F. A., Am. Mineralogist 22, 561 (1937). 2/. Novácek, R., Am. Mineralogist 24, 317 (1939).

3) Jaffe, H. W., Sherwood, A. M., and Peterson, M. J., Am. Mineralogis $33,152-157$ (1948).

SKIODOWSKITE $\mathrm{Mg}\left(\mathrm{UO}_{2}\right)_{2} \mathrm{Si}_{2} \mathrm{O}_{7} \cdot \mathrm{7H}_{2} \mathrm{O}$

Specimen Data

Mineral and identi- Location

fying number

Sklodowskite

USNM 105130

Sklodowskite

USNM R6700

Skindowskite

HNDI 86773

Phase 81

Katanga,

Katanga,

Katanga,

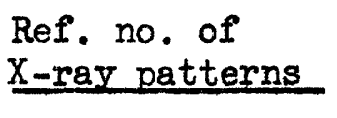

TE 627

Belgian Congo

Belgian Congo

Belgian Congo

Har. 1691
Quantity

USGS-X

USNM-20 g

USGS-XS

USNM- $3 \times 3$, L 
SODDYITE $5 \mathrm{UO}_{3} \cdot 2 \mathrm{SiO}_{2} \cdot 6 \mathrm{H}_{2} \mathrm{O}$ ?

Specimen Data

\begin{tabular}{|c|c|c|c|}
\hline $\begin{array}{l}\text { Mineral and identi- } \\
\text { fying number }\end{array}$ & Location & $\begin{array}{l}\text { Ref. no. of } \\
X \text {-ray patterns }\end{array}$ & Quantity \\
\hline $\begin{array}{l}\text { Soddyite } \\
\text { uSNM } 95601\end{array}$ & $\begin{array}{l}\text { Chinkolobwe mine, } \\
\text { Katanga, Belgian Congo }\end{array}$ & --- & $\begin{array}{l}\text { USGS-XS } \\
\text { USMM-100 g }\end{array}$ \\
\hline $\begin{array}{l}\text { Soddyite } \\
\text { USNM C4513 }\end{array}$ & $\begin{array}{l}\text { Chinkolobwe mine, } \\
\text { Katanga, Belgian Congo }\end{array}$ &.-- & $\begin{array}{l}\text { USGS-X } \\
\text { USNM-50 g }\end{array}$ \\
\hline $\begin{array}{l}\text { Soddyite } \\
\text { USNM R5896 }\end{array}$ & $\begin{array}{l}\text { Chinkolobwe mine, } \\
\text { Katanga, Belgian Congo }\end{array}$ & $-\cdots$ & $\begin{array}{l}\text { USGS-XS } \\
\text { USNM-50 g }\end{array}$ \\
\hline $\begin{array}{l}\text { Soddyite } \\
\text { HMM } 86774\end{array}$ & $\begin{array}{l}\text { Chinkolobwe mine, } \\
\text { Katanga, Belgian Congo }\end{array}$ & $\begin{array}{l}\text { TE } 3162 \\
\text { Har. } 1194\end{array}$ & USGS-XS \\
\hline $\begin{array}{l}\text { Soddyite } \\
\text { HMM } 86775\end{array}$ & $\begin{array}{l}\text { Chinkolobwe mine, } \\
\text { Katanga, Belgian Congo }\end{array}$ & TE 3168 & USGS-S \\
\hline
\end{tabular}

SWARTZITE $\mathrm{CaMgUO}_{2}\left(\mathrm{CO}_{3}\right)_{3} \cdot \mathrm{I2H}_{2} \mathrm{O}$

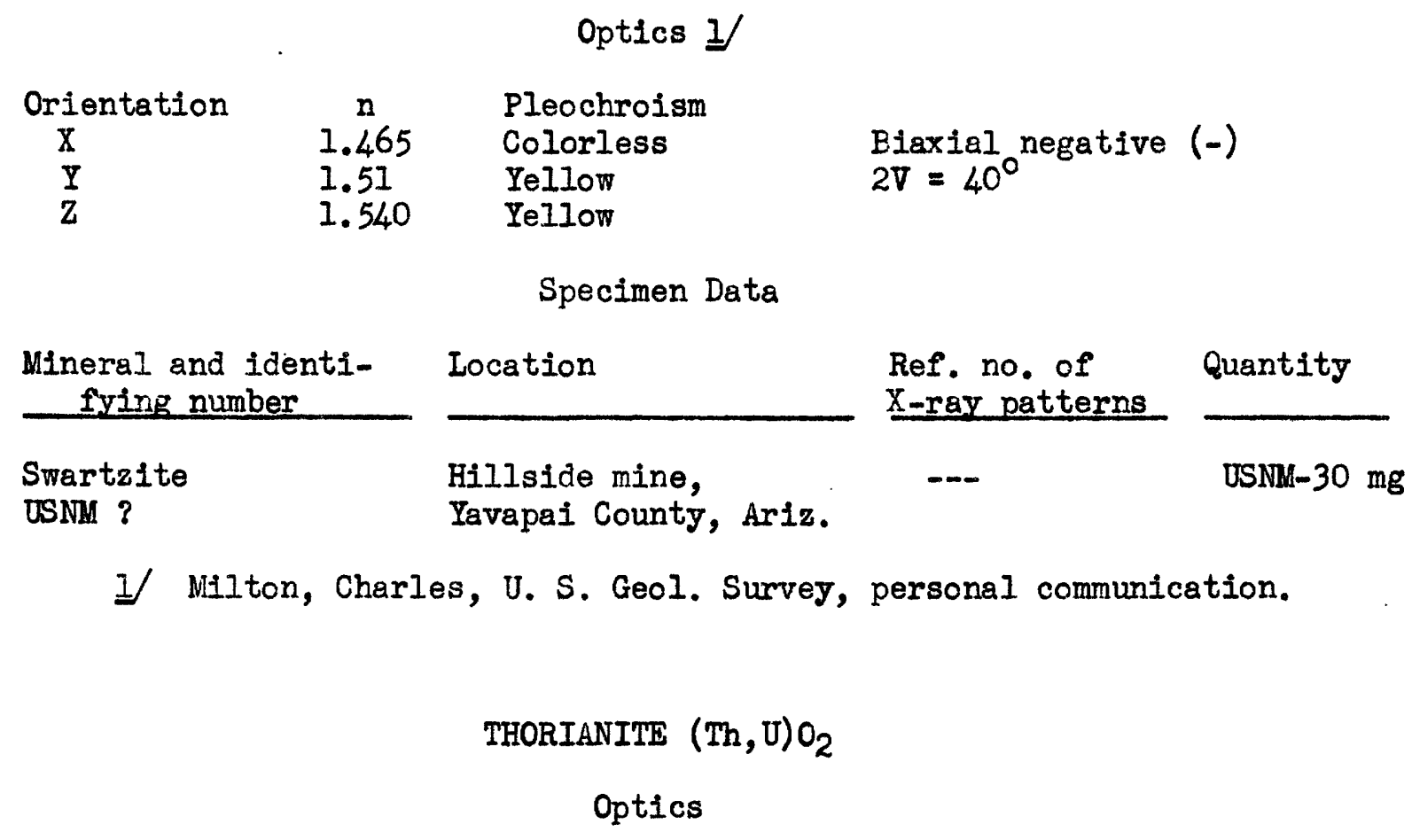

Transparent in very thin splinters. Isotropic; $\mathrm{n}$ variable, averages $2.20 \pm .1 /$ 
Thorianite (Cont.)

Specimen Data

\begin{tabular}{|c|c|c|c|}
\hline $\begin{array}{l}\text { Minersl and identi- } \\
\text { fying number }\end{array}$ & Location & $\begin{array}{l}\text { Ref. no. of } \\
\text { X-ray patterns }\end{array}$ & Quantity \\
\hline $\begin{array}{l}\text { Thorianite } \\
\text { TE }\end{array}$ & $\begin{array}{l}\text { Buckland-Siwalik area, } \\
\text { Seward Peninsula, Alaska }\end{array}$ & TE 286 & -- \\
\hline $\begin{array}{l}\text { Thorianite } \\
\text { USNM 93295 }\end{array}$ & $\begin{array}{l}\text { Bambaraluma Valley, } \\
\text { Ceylon }\end{array}$ & TE 579 & $\begin{array}{l}\text { USCS-XS } \\
\text { USNM-30 g }\end{array}$ \\
\hline $\begin{array}{l}\text { Thorianite } \\
\text { USNM } 96863\end{array}$ & Ceylon &.-- & $\begin{array}{l}\text { USGS-XS } \\
\text { USNM-200 }\end{array}$ \\
\hline
\end{tabular}

Uranothorianite--Uranium-rich member of series

Uranothorianite USNM R3679
Landbo, Norway

I) Larsen, E. S., U. S. Geol. Survey Bull. 679, 143 (1921).
TE 263

Ignited

USNM-15 g

THORITE ThSiO 4

Specimen Data
Mineral and ident1-
Location
Ref. no. of
X-ray patterns
Thorite
USNM R7901
Stove Mountain,
-.-
Quantity
fying number
El Paso County, Colo.
Thorite
USNM R7040
Lindvikkollen,

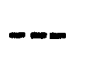
Kragero, Norway
USGS-XS
USNM-30 $\mathrm{g}$.
Thorite
HMM 85827
Arendal,
Har. 11321
Not ignited,
Norway
USGS-XS
USNM-50 $\mathrm{g}$
no pattern
Auerlite--Phosphatian variety of thorite
Auerlite
USNM R3684
Henderson County,
USGS-XS
N.C.
USNM-10 g 
Thorite----Specimen Data (Cont.)

Calciothorite--Variety of thorite

\begin{tabular}{|c|c|c|c|}
\hline $\begin{array}{l}\text { Mineral and identi- } \\
\text { fying number }\end{array}$ & Location & $\begin{array}{l}\text { Ref. no. of } \\
\text { X-ray patterns }\end{array}$ & Quantity \\
\hline $\begin{array}{l}\text { Calciothorite } \\
\text { USNM R3681 }\end{array}$ & $\begin{array}{l}\text { Arö, Langesund Fiord, } \\
\text { Norway }\end{array}$ & $\begin{array}{l}\text { TI } 720 \\
\text { Ignited }\end{array}$ & $\begin{array}{l}\text { USGS-XS } \\
\text { USNM-10 g }\end{array}$ \\
\hline \multicolumn{4}{|c|}{ Enalite--Uranoan thorite } \\
\hline $\begin{array}{l}\text { Enalite } \\
\text { USNM } 105392\end{array}$ & $\begin{array}{l}\text { Fukuoke-mura, Enagun, } \\
\text { Gita Prefectu, Japan }\end{array}$ & TE 578 & $\begin{array}{l}\text { USGS-X } \\
\text { USNM-5 g }\end{array}$ \\
\hline \multicolumn{4}{|c|}{ Eucrasite--Variety of thorite } \\
\hline $\begin{array}{l}\text { Eucrasite } \\
\text { USNM R3682 }\end{array}$ & $\begin{array}{l}\text { Brevig, } \\
\text { Norway }\end{array}$ & $\begin{array}{l}\text { TE } 719 \\
\text { Ignited }\end{array}$ & $\begin{array}{l}\text { USGS-XS } \\
\text { USMM-10 g }\end{array}$ \\
\hline \multicolumn{4}{|c|}{ Ferrothorite- A ferrian thorite } \\
\hline $\begin{array}{l}\text { Ferrothorite } \\
\text { USNM } 97278\end{array}$ & $\begin{array}{l}\text { Befaritra, } \\
\text { Madagascar }\end{array}$ & -- & USNM-25 g \\
\hline \multicolumn{4}{|c|}{ Freyalite--Variety of thorite } \\
\hline $\begin{array}{l}\text { Freyalite } \\
\text { USNM R3683 }\end{array}$ & $\begin{array}{l}\text { Langesund Fiord, } \\
\text { Norway }\end{array}$ & --- & $\begin{array}{l}\text { USGS-XS } \\
\text { USNM-10 g }\end{array}$ \\
\hline \multicolumn{4}{|c|}{ Orangite--Synonym of thorite } \\
\hline $\begin{array}{l}\text { Orangite } \\
\text { USNM R3678 }\end{array}$ & $\begin{array}{l}\text { Brevig, } \\
\text { Norway }\end{array}$ & $\begin{array}{l}\text { Inv, } 2573 \\
\text { Ignited }\end{array}$ & USNM-5 $\mathrm{g}$ \\
\hline $\begin{array}{l}\text { Orangite } \\
\text { usNM R7043 }\end{array}$ & $\begin{array}{l}\text { Laaven, Langesund, } \\
\text { Brevig, Norway }\end{array}$ & --- & $\begin{array}{l}\text { USGS-XS } \\
\text { USNM-50 g }\end{array}$ \\
\hline Orangite & $\begin{array}{l}\text { Brevig, } \\
\text { Norway }\end{array}$ & $\begin{array}{l}\text { Inv, } 2590 \\
\text { Ignited }\end{array}$ & -- \\
\hline \multicolumn{4}{|c|}{ Uranothorite-- Uranoan thorite } \\
\hline $\begin{array}{l}\text { Uranothorite } \\
\text { USMM R3680 }\end{array}$ & $\begin{array}{l}\text { Arö, } \\
\text { Norway }\end{array}$ & $\begin{array}{l}\mathrm{TE} 724 \\
\text { Ignited }\end{array}$ & USNM-10 g \\
\hline
\end{tabular}


THOROGUMMITE $(\mathrm{Th}, \mathrm{U}) \mathrm{S}_{4} \mathrm{O}_{4} \cdot 6 \mathrm{H}_{2} \mathrm{O}$ ?

Specimen Data

\begin{tabular}{|c|c|c|c|}
\hline $\begin{array}{l}\text { Mineral and identi- } \\
\text { fring number }\end{array}$ & Location & $\begin{array}{l}\text { Ref. no. of } \\
\text { X-ray patterns }\end{array}$ & Quantity \\
\hline $\begin{array}{l}\text { Thorogummite } \\
\text { USNM C4510 }\end{array}$ & $\begin{array}{l}\text { Llano County, } \\
\text { Texas }\end{array}$ & -- & $\begin{array}{l}\text { USGS-XS } \\
\text { USNM-50 g }\end{array}$ \\
\hline $\begin{array}{l}\text { Thorogummite } \\
\text { USNM } 104045\end{array}$ & $\begin{array}{l}\text { Iisaka, Fukushima, } \\
\text { Japan }\end{array}$ & -- & $\begin{array}{l}\text { USGS-XS } \\
\text { USNM-10 g }\end{array}$ \\
\hline $\begin{array}{l}\text { Thorogummite } \\
\text { USNM } 95959\end{array}$ & $\begin{array}{l}\text { Wodgina, } \\
\text { West Australia }\end{array}$ & $\begin{array}{l}\text { TE } 705 \\
\text { Ignited } \\
\text { Modified ignit } 706 \\
\text { Modified }\end{array}$ & USGS-XS \\
\hline
\end{tabular}

THOROTUNGSTITE - Essentially a basic tungstate (or oxide) with $(\mathrm{Al}, \mathrm{Fe}):(\mathrm{Th}, \mathrm{Ca}, \mathrm{Ce}, \mathrm{Zr}): \mathrm{W} \sim 1: 1: 3$

Optics

Optically negative $(-)$ with Indices over 1.74 and strong birefringence. Specimen Data
Mineral and identi-
fying number
Location
Ref. no. of
X-ray patterns
Quantity
Thorotungstite
USNM 95659
Pula1, Kinta,
Federated Malay States
TE 565
USGS-XS
USNM-300 g
Thorotungstite I/
Pulai, Kinta,
Federated Malay States
USNM-300 $\mathrm{g}$
USNM 96854
I/ Scrivenor, J. B., Am. Jour. Sc1., 5th ser., 13, 487-490 (1927).

$$
\text { TORBERNITE } \mathrm{Cu}\left(\mathrm{UO}_{2}\right)_{2}\left(\mathrm{PO}_{4}\right)_{2} \cdot 8-12 \mathrm{H}_{2} \mathrm{O}
$$
Optics

$\begin{array}{lcll} & \text { n Cornwall } & \text { Dichroism } & \\ 0 & 1.592 & \text { Sky blue } & \text { Uniaxial negative (-). } \\ \mathrm{E} & 1.582 & \text { Green } & \end{array}$


Torbernite----Optics (Cont.)

The indices of refraction and the specific gravity doubtless vary with water content in the range from 8 to $12 \mathrm{H}_{2} \mathrm{O}$ but correlated data are lacking. Host of the published optical data on so-called torbernite are of uncertain affiliation and may belong to metatorbernite.

Specimen Data

Mineral and identi- Location

fying number

Torbernite

$\mathrm{TE}$

Torbernite

$\mathrm{TE}$

Torbernite

USNM 97445

Torbernite

HMM 62582

Torbernite

USNM 87239

Torbernite

USNM R5670

Torbernite

USNM R8385

Torbernite

USNM 94782

Torbernite

USNM 105126

Torbernite

USNM $\mathrm{C}_{4517}$

Torbernite

USNM 101995

Torbernite

USNM C4382

Phase 13

Bohemia

Katanga,

Buhowo,

Bulgaria
Ref. no. of X-ray patterns

Robineaur claim, near Lawson, Colo.

Majuba Hill, Nev.

Spruce Pine, N.C.

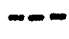

USGS-XS

USNMI-9x $15, M$

Mount Painter,

Australia

Mount Painter,

Australia

USGS-XS

USNM-20 g

Joachimsthal,

Chinkolobwe, Katanga

Belgian Congo

Belgian Congo

Belgian Congo

Chinkolobwe, Katanga,

Belgian Congo

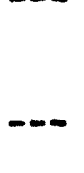

TE 3109

HMM-75 g

USGS-XS

USNM-9 $15, M$

USGS-XS

USNM-5 g

USGS-X

USNM-50 $\mathrm{g}$

USGS-XS

USNM-10 g

USGS-X

USNM-10 $\mathrm{g}$

USGS-XS

USNM-5 $\mathrm{g}$

Cornwall, England

USNM-3x6,M 
Torbernite---Specimen Data (Cont.)

\begin{tabular}{|c|c|c|c|}
\hline $\begin{array}{l}\text { Mineral and ident1- } \\
\text { fying number }\end{array}$ & Iocation & $\begin{array}{l}\text { Ref. no. of } \\
X \text {-rey patterns }\end{array}$ & Quantity \\
\hline $\begin{array}{l}\text { Torbernite } \\
\text { USNM C4383 }\end{array}$ & $\begin{array}{l}\text { Redruth, } \\
\text { Cornwall, England }\end{array}$ & -- & $\begin{array}{l}\text { USGS-XS } \\
\text { USMM-5 g }\end{array}$ \\
\hline $\begin{array}{l}\text { Torbernite } \\
\text { mMa } 7112 \\
\text { Phase } 13\end{array}$ & $\begin{array}{l}\text { Old Gunnis Lake, } \\
\text { Calstock, } \\
\text { Cornwall, England }\end{array}$ & Har. 3926 & HMM-6×6,I \\
\hline
\end{tabular}

$$
\begin{gathered}
\text { TRÖGERITE }\left(\mathrm{UO}_{2}\right)_{3}\left(\mathrm{AsO}_{4}\right)_{2} \cdot 12 \mathrm{H}_{2} \mathrm{O} \\
\text { Optics }
\end{gathered}
$$

Uniaxial or biaxial (-). In transmitted light pale lemon yellow.

\begin{tabular}{|c|c|c|c|}
\hline $\begin{array}{l}\text { Orientation I/ } \\
\underset{X}{Y} \quad c \\
\underset{2 V}{2 \Lambda_{a}=12^{\circ}-14^{\circ}}\end{array}$ & $\begin{array}{l}\quad \mathrm{n} 2 / \\
1.585 \pm .0 .005 \\
1.630 \pm 0.005 \\
1.630 \pm 0.005 \\
\text { Very smaii }\end{array}$ & $\begin{array}{l}1.580 \\
1.624 \\
0^{0}\end{array}$ & \\
\hline \multicolumn{4}{|c|}{ Specimen Data } \\
\hline $\begin{array}{l}\text { Mineral and identi- } \\
\text { fying number }\end{array}$ & Location & $\begin{array}{l}\text { Ref. no. of } \\
X \text {-ray patterns }\end{array}$ & Quantity \\
\hline $\begin{array}{l}\text { Trögerite } \\
\text { USNM C4394 }\end{array}$ & $\begin{array}{l}\text { Schneeberg, } \\
\text { Saxony }\end{array}$ & -- & $\begin{array}{l}\text { USGS-X } \\
\text { USNM-3×2,I }\end{array}$ \\
\hline $\begin{array}{l}\text { Trögerite } \\
\text { USNM R5683 }\end{array}$ & $\begin{array}{l}\text { Weisser-Hirch mine, } \\
\text { Schneeberg, Saxony }\end{array}$ & --- & $\begin{array}{l}\text { USGS-X } \\
\text { USNM- } 6 \times 9,1\end{array}$ \\
\hline $\begin{array}{l}\text { Trögerite } \\
\text { USNM R7927 }\end{array}$ & $\begin{array}{l}\text { Schneeberg, } \\
\text { Saxony }\end{array}$ & TE 3131 & USGS-6x6, I \\
\hline
\end{tabular}
Not pleochroic.

I/ Goldscrmidt, V., Zeitschr. Kristallographie, 31, 468 (1899).

2) Larsen, 3. S., U. S. Geol. Survey Bull. 679, 145 (1921). 


\section{TYUYAMUNITE $\mathrm{Ca}\left(\mathrm{UO}_{2}\right)_{2}\left(\mathrm{VO}_{4}\right)_{2} \cdot \mathrm{nH}_{2} \mathrm{O}$ \\ ( $n=9-10$, but may be down to 4 ) \\ Optics}

In transmitted light, colorless to pale yellow. Faintly pleochroic, $X$ nearly colorless, $I$ pale canary yellow, $Z$ canary yellow. $I$

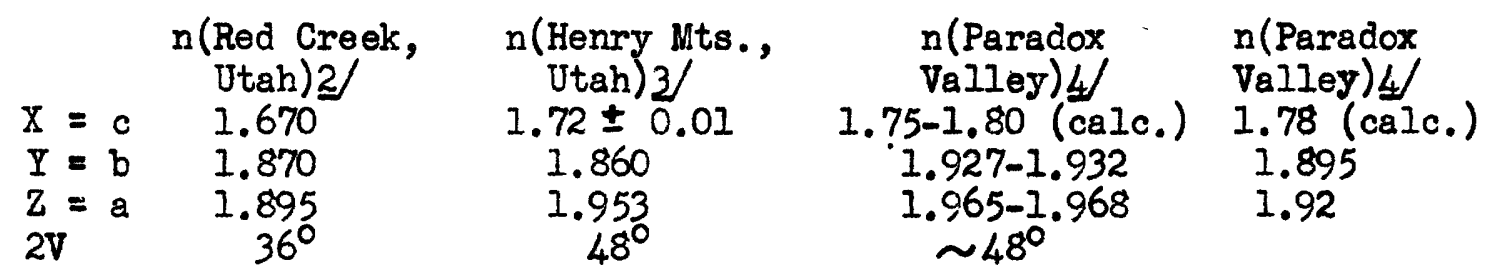

Specimen Data

\begin{tabular}{|c|c|c|c|}
\hline $\begin{array}{l}\text { Mineral and identi- } \\
\text { fying number }\end{array}$ & Location & $\begin{array}{l}\text { Ref. no. of } \\
\text { X-ray patterns }\end{array}$ & Quantity \\
\hline $\begin{array}{l}\text { Tyuyamunite } \\
\text { HMM } 80815\end{array}$ & $\begin{array}{l}\text { Long Park, } \\
\text { Montrose County, Colo. }\end{array}$ & TE 3063 & --- \\
\hline $\begin{array}{l}\text { Tyuyamunite } \\
\text { USNM } 97070\end{array}$ & $\begin{array}{l}\text { Long Park, } \\
\text { Montrose County, Colo. }\end{array}$ & Inv. 4166 & USMM-300 g \\
\hline $\begin{array}{l}\text { Tyuyamunite } \\
\text { HMM TCC }\end{array}$ & $\begin{array}{l}\text { Mauch Chunk, } \\
\text { Pa. }\end{array}$ & TE 3102 & --- \\
\hline $\begin{array}{l}\text { Tyuyamunite. } \\
\text { USNM } 96159\end{array}$ & $\begin{array}{l}\text { Yellow Cat district, } \\
\text { Grand County, Utah }\end{array}$ & -- & USNM $300 \mathrm{~g}$ \\
\hline $\begin{array}{l}\text { Tyuyamunite } \\
\text { USNM } 96054\end{array}$ & $\begin{array}{l}\text { Yellow Cat Wash, } \\
\text { Grand County, Utah }\end{array}$ & -- & $\begin{array}{l}\text { USGS-X } \\
\text { USNM-300 g }\end{array}$ \\
\hline $\begin{array}{l}\text { Tyuyamunite } \\
\text { USNM } 95387\end{array}$ & $\begin{array}{l}\text { Fergana, Russian } \\
\text { Turkestan }\end{array}$ & --- & $\begin{array}{l}\text { USGS-XS } \\
\text { USNM-5 g }\end{array}$ \\
\hline $\begin{array}{l}\text { Tyuyamunite } \\
\text { USNM R5697 }\end{array}$ & $\begin{array}{l}\text { Fergana, Russian } \\
\text { Turkestan }\end{array}$ & Inv. 4172 & -- \\
\hline $\begin{array}{l}\text { Tyuyamunite } \\
\text { HMM } 89539\end{array}$ & $\begin{array}{l}\text { Fergana, Russian } \\
\text { Turkestan }\end{array}$ & Har. 4196 & $H M M-\frac{1}{2} g$ \\
\hline
\end{tabular}

I/ Khlopin, V. G., Acad. Russie, 73, (1925) and Dolivo-Dobrovolsky, v. V., Soc. russe minéralogie Mém. 2, 54, 359 (1925).

$2 /$ Larsen. F. S., U. S. Geol. Survey Buil. 679, 148 (1921).

3) Ross, C. S., in Hess, F. L., U. S. Geol. Survey Bull. 750-D, 73 (1925).

4/ Merwin, H. E., in Hillebrand, W. F., Am. Jour. Sci, 5th ser., 8, 201 (1924). 
URACONITE - Hydrous sulfate of $\mathrm{U}$ and $\mathrm{Cu}$.

Specimen Data

\begin{tabular}{|c|c|c|c|}
\hline $\begin{array}{l}\text { Mineral and identi- } \\
\text { fying number }\end{array}$ & Location & $\begin{array}{l}\text { Ref. no, of } \\
\text { X-ray patterns }\end{array}$ & Quantity \\
\hline $\begin{array}{l}\text { Uraconite } \\
\text { USNM C4769 }\end{array}$ & $\begin{array}{l}\text { Telegraph mine, } \\
\text { Gilpin County, Colo. }\end{array}$ & TE 300 & $\begin{array}{l}\text { USGS-X } \\
\text { USNMI- } 3 \times 6, I\end{array}$ \\
\hline $\begin{array}{l}\text { Uraconite } \\
\text { HMM } 69071 \\
\text { Phase } 65\end{array}$ & $\begin{array}{l}\text { Joachimsthal, } \\
\text { Eohemia }\end{array}$ & Har. 1431 & $\operatorname{HMM}-3 \times 3, L$ \\
\hline $\begin{array}{l}\text { Uraconite } \\
\text { USNM } 93341\end{array}$ & $\begin{array}{l}\text { St. Just, Cornwall, } \\
\text { England }\end{array}$ & $-\infty$ & $\begin{array}{l}\text { USGS-X } \\
\text { USNM-3x9,I }\end{array}$ \\
\hline $\begin{array}{l}\text { Uraconite } \\
\text { USNM R6767 }\end{array}$ & $\begin{array}{l}\text { Daniel mine, } \\
\text { Schneeberg, Saxony }\end{array}$ & -- & $\begin{array}{l}\text { USGS-XS } \\
\text { USNM-9x15,I }\end{array}$ \\
\hline
\end{tabular}

URANINITE $\mathrm{UO}_{2}$ (between $\mathrm{UO}_{2}$ and $\mathrm{U}_{3} \mathrm{O}_{8}$, with $\mathrm{U}^{4}$ predominant) Optics

In transmitted light, greenish, yellowish, or deep brown. Usually opaque.

$$
\text { Specimen Data }
$$

\begin{tabular}{|c|c|c|c|}
\hline $\begin{array}{l}\text { Mineral and ident1- } \\
\text { fying number }\end{array}$ & Iocation & $\begin{array}{l}\text { Ref. no. of } \\
\text { X-ray patterns }\end{array}$ & Quantity \\
\hline $\begin{array}{l}\text { Uraninite } \\
\mathrm{TE}\end{array}$ & Eldorado mine, Colo. & $-\cdots$ & USGS-XSC \\
\hline $\begin{array}{l}\text { Uraninite } \\
\text { HMM 90884 }\end{array}$ & Newry, Maine & Har. 3865 & --- \\
\hline $\begin{array}{l}\text { Uraninite } \\
\text { USNM 102618 }\end{array}$ & $\begin{array}{l}\text { Ruggles mine, } \\
\text { Grafton, N.H. }\end{array}$ & -- & $\begin{array}{l}\text { USGS-XS } \\
\text { USNM-500 g }\end{array}$ \\
\hline $\begin{array}{l}\text { Uraninite } \\
\text { USNM C5838 }\end{array}$ & $\begin{array}{l}\text { Ruggles mine, } \\
\text { Grafton, N.H. }\end{array}$ & --- & $\begin{array}{l}\text { USGS-XS } \\
\text { USNM-1200 }\end{array}$ \\
\hline $\begin{array}{l}\text { Uraninite } \\
\text { TE }\end{array}$ & $\begin{array}{l}\text { Katanga, Belgian } \\
\text { Congo }\end{array}$ & -- & USGS-XSC \\
\hline $\begin{array}{l}\text { Uraninite } \\
\text { USNM R8383 }\end{array}$ & $\begin{array}{l}\text { Kalongwe, } \\
\text { Belgian Congo }\end{array}$ & $\cdots$ & $\begin{array}{l}\text { USGS-XS } \\
\text { USMM-100 } \mathrm{g}\end{array}$ \\
\hline
\end{tabular}


Uraninite---Specimen Data (Cont.)

Mineral and identi- Location

Ref. no. of

X-ray patterrs

fying number

Uraninite

USNMI R8391

Uraninite

USNR: R840I

Uraninite

USNM C2257

Uraninite

USNM 91225

Uraninite $1 /$

USNM 83570

Uraninite

USNM 103596

Broggerite

USNM C4497

Cleveite

USNM 95469

Nivenite

USNM $\mathrm{C} 4510$

Nivenite

USNM R5878

Nivenite

USNM R5888
Kalongwe, Katanga,

Belgian Congo

Chinkolobwe, Katanga

Belgian Congo

Joachimsthal,

Bohemia

Brejaúba, Minas

Geraes, Brazil

Huggeneskilen,

Moss, Norway

Gordonia, West Cape

Coloisy, South Africa

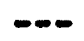

$--$

$-\cdots$

$-\cdots$

$\operatorname{TE} 3098$

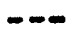

Bröggerite--Thorian uraninite $(\mathrm{U}, \mathrm{Th}) \mathrm{O}_{2}$

Raade,

Norway
USCSS-XS

USNM-25 g

Cleveite--Uraninite with rare earths

Rosland, near

Tvedestrand, Norway

$--$

USGS-XS

USNM-75 g

Nivenite--Uraninite with rare earths

Llano County,

Texas

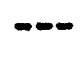

Llano County,

Texas

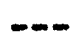

Joachimsthal,

$--$

Bohemia
USGS-XS

USNM-75 g

USGS-XS

USNM-50 g

USGS-XS

USNM-20 $\mathrm{g}$
USGS-X

USNM-50 $g$

USGS-XS

USNAI-1000

USGS-XS

USNM-5 $\mathrm{g}$.

USGS-X

USNM-300 $\mathrm{g}$

USNM-10 g

USGS-XS

USNM-4000 g

Pitchblende--Colloform and massive uraninite

- Pitchblende TE
Central City

area, Colo.

USGS-XS 
Pitchblende---Specimen Data (Cont.)

\begin{tabular}{|c|c|c|c|}
\hline $\begin{array}{l}\text { Mineral and identi- } \\
\text { fying number }\end{array}$ & Location & $\begin{array}{l}\text { Ref. no. of } \\
\text { X-ray patterns }\end{array}$ & Quantity \\
\hline $\begin{array}{l}\text { Pitchblende } \\
\mathrm{TE}\end{array}$ & Caribou mine, Colo. & $-\infty$ & USGS-XSC \\
\hline $\begin{array}{l}\text { Pitchblende } \\
\text { TE }\end{array}$ & Prairie Divide, Colo. & -- & USGS-XS \\
\hline $\begin{array}{l}\text { Pitchblende } \\
\text { USNM } 101917\end{array}$ & $\begin{array}{l}\text { Deer Park no. } 5 \text { mine, } \\
\text { Penland, N.C. }\end{array}$ & TE 566 & $\begin{array}{l}\text { USGS-XS } \\
\text { USNM-15 g }\end{array}$ \\
\hline $\begin{array}{l}\text { Pitchblende } \\
\text { USNM R } 4339\end{array}$ & Spruce Pine, N.C. & TE 560 & $\begin{array}{l}\text { USGS-XS } \\
\text { USNM-100 g }\end{array}$ \\
\hline $\begin{array}{l}\text { Pitchblende } \\
\text { USNM } 104784\end{array}$ & $\begin{array}{l}\text { Chinkolobwe, Katanga, } \\
\text { Belgian Congo }\end{array}$ & TE 572 & $\begin{array}{l}\text { USGS-XS } \\
\text { USNM-200 g }\end{array}$ \\
\hline $\begin{array}{l}\text { Pitchblende } \\
\text { USNM } 104838\end{array}$ & $\begin{array}{l}\text { Columbia, South } \\
\text { America }\end{array}$ & $\begin{array}{l}\text { TE } 709 \\
\text { Ignited } \\
\text { TE } 710, \text { TE } 712 \\
\text { Modified Ignitic }\end{array}$ & $\begin{array}{l}\text { USGS-XS } \\
\text { USNM-500 g } \\
\text { on }\end{array}$ \\
\hline
\end{tabular}

Uranopissite--Synonym of uraninite

Uranopissite

USMM R5881
Przibram,

Bohemia
TE 558

USGS-XS

USNM-300 $\mathrm{g}$

I/ Hillebrend, W. F., in Clarke, F. W., U. S. Geol. Survey Bull. 78, 48 (1891).

URANOCIRCITE Ba $\left(\mathrm{UO}_{2}\right)_{2}\left(\mathrm{PO}_{4}\right)_{2} \cdot 8 \mathrm{H}_{2} \mathrm{O}$

Optics.

Orientation
$X \quad a$
$Y$
$Z$
$2 V$ $\mathrm{n}$ (Synthetic) 2/

1.604

$1.610 \pm 0.003$

$1.622 \pm 0.003$

$1.623 \pm 0.003$

Small
Pleochroism

Nearly colorless

Pale canary yellow

Pale canary yellow

Biaxial (-) 
Uranocircite (Cont.)

Specimen Data

Mineral and identi-
fying number

Uranocircite

AMNH 15779

Uranocircite

USNM R5687

Uranocircite

HMM 96586

Phase 96

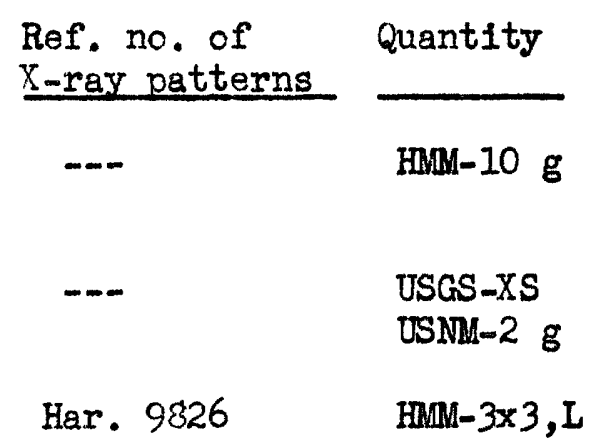

Ref. no. of

Location

Falkenstein,

Saxony

Bergen, near

Falkenberg, Saxony

Falkenstein, Saxony
USGS-XS

USNM-2 $\mathrm{g}$

HMM- $3 \times 3, \mathrm{~L}$

If Larsen, E. S., U. S. Geol. Survey Bull. 679, 149 (1921).

2/ Fairchild, J.'G., Am. Mineralogist 14, 265 (1929).

URANOPHANE $\mathrm{Ca}\left(\mathrm{UO}_{2}\right)_{2} \mathrm{Si}_{2} \mathrm{O}_{7} \cdot 6 \mathrm{H}_{2} \mathrm{O}$

Specimen Data

Mineral and identi-

fying number

Uranophane

USNM 102618

Uranophane

USNM 96481

Uranophane

USNM C5415

Uranophane

USNM 103189

Uranophane

$\mathrm{TE}$

Uranophane

USNM R4838

Uranophane

HMM 89756

Uranophane

HMM 87092
Location

Ruggles mine,

Grafton, N.H.

Fannie Gouge mine,

Spruce Pine, N.C.

Spruce Pine, N.C.

Spruce Pine, N.C.

TE 600

Lusk, Wyo.

$--$

Inv. 5086

Victoria, Australia

Ref. no. of
X-ray patterns

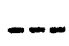

$\therefore$

$--$

Wölsenberg,

Bavaria

Katanga, Belgian

Congo
Quantity

USGS-X

USNM-9x $12, M$

USGS-XS

USNM- $6 \times 9$, I

USGS-XS

USNM-6x9,I

USGS-X

USNM-6x 9, I

USGS-XS

USGS-S

USNM-9x9, L

HBM-9x $12, L$

HMM-25 g 
Uranophane----Specimen Data (Cont.)

\begin{tabular}{|c|c|c|c|}
\hline $\begin{array}{l}\text { Mineral and identi- } \\
\text { fying number }\end{array}$ & Location & $\begin{array}{l}\text { Ref. no. of } \\
X \text {-ray patterns }\end{array}$ & Quantity \\
\hline $\begin{array}{l}\text { Uranophane } \\
\text { USNM } 91225\end{array}$ & $\begin{array}{l}\text { Brejaúba, Minas } \\
\text { Geraes, Brazil }\end{array}$ & -- & $\begin{array}{l}\text { USGS-X } \\
\text { USNM- } 5 \times 8, L\end{array}$ \\
\hline
\end{tabular}

Beta-uranophane--Polymorph of uranophane

Optics I]

$\begin{array}{cllll}\text { Orientation } & n(\mathrm{Na}) & \mathrm{n}(\mathrm{Na}) & \text { Pleochroism } & \\ \mathrm{X} & 1.665 & 1.662 & \text { Nearly colorless } & \text { Biaxial } \\ \mathrm{Y} & 1.686 & 1.686 & \text { Deep yellow } & 2 \mathrm{~V} \text { large } \\ \mathrm{Z} & 1.696 & 1.694 & \text { Deep yellow } & \begin{array}{l}\mathrm{r}>\mathrm{v}, \text { crossed, } \\ \text { strong }\end{array}\end{array}$

Specimen Data Mineral and identi-
fying number

Beta-uranophane

HMM 90891

Phase 44

Beta-uranophane

USNM R2765

Beta-uranophane HMM 87644

Phase 7
Ref. no. of X-ray patterns

Newry, Maine

Frankfort, Philadelphia, Pa.

Joachimsthal, Bohemia
Har.

Quantity

Han $6 \times 6, \mathrm{~L}$

Inv. 2845

Har. 4172

Lambertite--Synonym of uranophane

Lambertite

USNM R5903
Lusk, Wyo.

$--+$

USGS-XS

USNM-9x6,I

Uranctil--Synonym of uranophane

Uranotin

Uranotil

HMM 87646
Luiswishi, Katanga,

Belgian Congo

Kuffersberg, Silesia
$--$

USGS-XS

Har. 3892

$H M M-2 g$

I/ Nová Yek, R., Česke SpoI. Nauk. Věstnik Article VII, 1-36 (1935). 


\section{URANOPIIITE $\left(\mathrm{UO}_{2}\right)_{6}\left(\mathrm{SO}_{4}\right)(\mathrm{OH})_{10} \cdot 12 \mathrm{H}_{2} \mathrm{O}$}

OptIcs

Blaxial ( $t), 2 \mathrm{~V}$ rather large $(\mathrm{Na}), 0^{\circ}$ for some wave lengths. In transmitted light, colorless to pale yellon. Not perceptibly pleochroic.

$\begin{array}{ccll}\text { Orfientation } & n \text { (Joachimsthal) }) & n(\mathrm{Na}) 2 / & \\ \mathrm{X} \sim \mathrm{b} & 1.621 & 1.623 & \mathrm{r}\langle\mathrm{v}, \text { extreme; } \\ \mathrm{Y} \wedge \mathrm{c}+18^{\circ} & 1.623 & 1.625 & \text { also } \mathrm{r}>\mathrm{v} . \\ \mathrm{Z} & 1.631 & 1.634 & \end{array}$

Specimen Data

\section{Mineral and identi- fying number}

Uranoplifte

ran 6074

Uranopilite

USNM R7876

Uranopilite

USNM R7929

Uranopilite

HAM 88035

Phase 76 llontrose Ccunty, Colo.

Huel Edwards, Cornwall, England

Joachinsthal, Germany

Johanngeorgenstadt, Saxony
Ref. no. of

X-ray patterns

Quantity

TE 604

USGS-X

USNM- $3 \times 3,1$

USGS-X

USII- $3 \times 3,2$

Har.

I/ Larsen, E. S., U. S. Geol. Survey BuIl. 679,150 ,(1921).

2) Novácek, R., Soc. royale sci. Eohème, CI. sci., Mém. 7 (1935) average of numerous measurements.

URANOSPHAERITE $\mathrm{Bi}_{2} \mathrm{~J}_{2} \mathrm{O}_{9} \cdot 3 \mathrm{H}_{2} \mathrm{O}$

Optics I I

Orientation

$\begin{array}{ll}\mathrm{X} & \mathrm{a} \\ \mathrm{Y} & \mathrm{b} \\ \mathrm{Z} & \mathrm{c}\end{array}$

n

$$
\begin{aligned}
& 1.955 \pm 0.01 \\
& 1.985 \pm 0.01 \\
& 2.05 \pm 0.01
\end{aligned}
$$

Biaxial nositive ( + )

2V large

Dispersion $r<v$, strong 
Uranosphaerite (Cont.)

Specimen Data

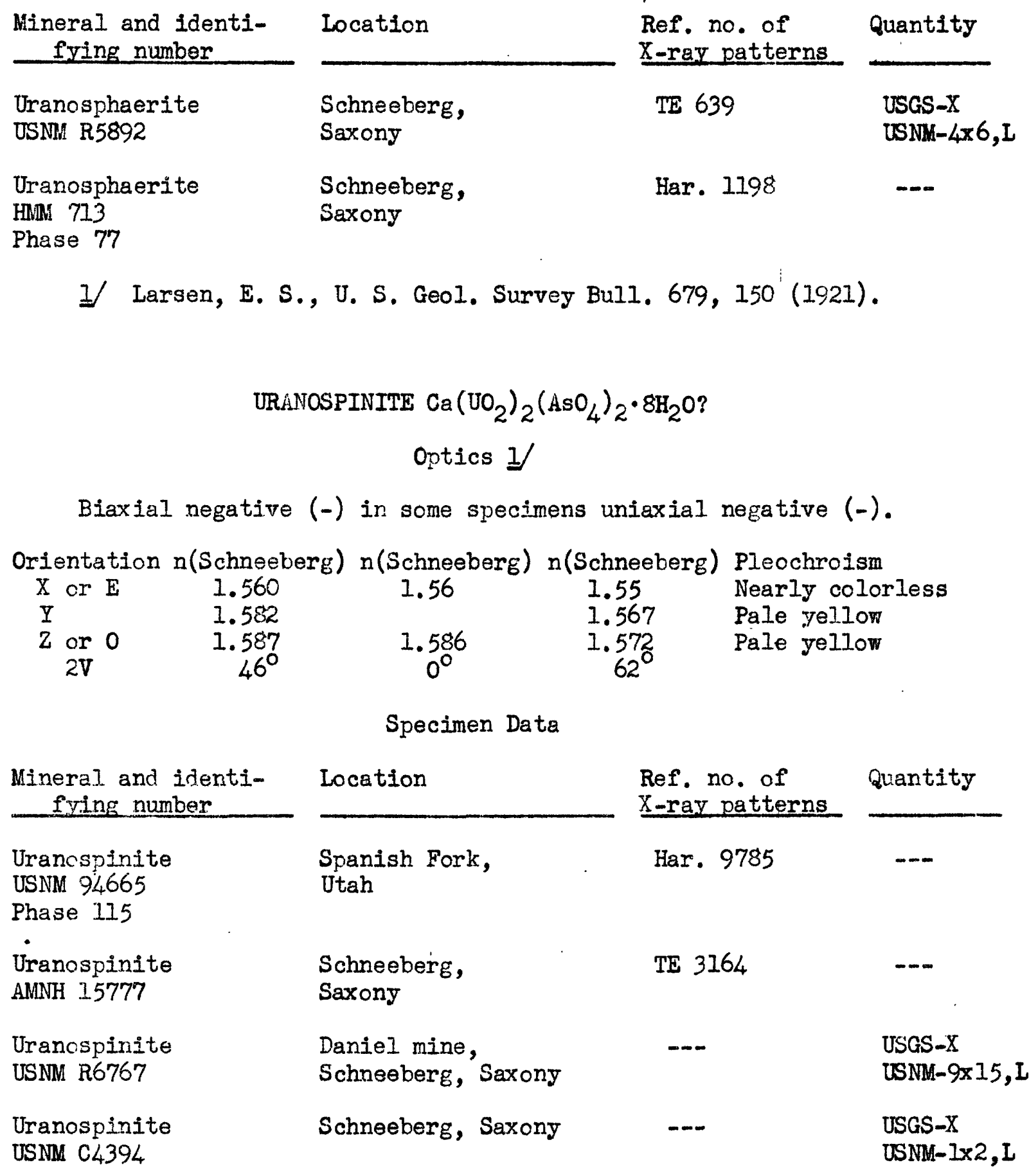


Uranospinite---Specimen Data (Cont.)

\begin{tabular}{llll}
$\begin{array}{l}\text { Mineral and identi- } \\
\text { fying number }\end{array}$ & Location & $\begin{array}{l}\text { Ref. no. of } \\
\text { X-ray patterns }\end{array}$ & Quantity \\
\cline { 1 - 1 } $\begin{array}{l}\text { Uranospinite } \\
\text { HMM }\end{array}$ & -- & Har. 8336 & $\cdots$ \\
$\begin{array}{l}\text { Uranospinite } \\
\text { HMM Phase 55 }\end{array}$ & $\begin{array}{l}\text { Neustådie, } \\
\text { Saxony }\end{array}$ & Har. 1594 & $\cdots$
\end{tabular}

1/, Larsen, E. S., U. S. Geol. Survey Bull. 679, 151 (1921). Goldschmidt, V., Zeitschr. Kristallographie 31, 468 (1899).

UVANITE $\mathrm{U}_{2} \mathrm{~V}_{6} \mathrm{O}_{21} \cdot 15 \mathrm{H}_{2} \mathrm{O}$

Optics

$\begin{array}{cc}\text { Orientation } & n \\ X & 1.817 \\ Y & 1.879 \\ Z & 2.057\end{array}$

Pleochroism Light brown Dark brown Greenish yellow

Biaxial positive (t) $2 V=52^{\circ}$

Specimen Data

Mineral and identi- Location

fying number

Uvanite I/

USNM R5708

Temple Rock,

Emery County, Utah

Ref. no. of

Quantity

X-ray patterns

Uvanite

HRM 62753

Temple Rock,

Emery County, Utah

TE 3132

Har. 1689

Har. 1597

USGS-XS

USNM-300 g

HMM-5 $\mathrm{g}$

Phase 2

I/ Hess, F. L., and Schaller, W. T., Washington Acad. Sci. Jour. 4, 576 (1914).

VANDENBRANDEITE $\mathrm{CuUO}_{4} \cdot 2 \mathrm{H}_{2} \mathrm{O}$

Optics

Green in transmitted light. Biaxial, apparently negative (-).I/ Absorption from green to colorless. 
Vandenbrandeite----Optics (Cont.)

$\begin{array}{clc} & n \text { (Kalongwe)2/ } & n \text { (Chinkolobwe) } 1 / \\ X & 1.77 \pm 0.02 & 1.76 \pm \\ Y & 1.78 \pm 0.02 & \\ Z & 1.80 \pm 0.02 & 1.80 \pm\end{array}$

Dispersion strong. 2V large. An optic axis is nearly $\perp\{001\}$. 21 elong. $=40^{\circ} \pm .1$

Specimen Data

\begin{tabular}{|c|c|c|c|c|}
\hline \multicolumn{2}{|c|}{$\begin{array}{l}\text { Mineral and identi- } \\
\text { fying number }\end{array}$} & Location & $\begin{array}{l}\text { Ref. no. of } \\
X \text {-ray patterns }\end{array}$ & Quantity \\
\hline \multicolumn{2}{|l|}{$\begin{array}{l}\text { Vandenbrandeite } \\
\text { USNM R8383 }\end{array}$} & $\begin{array}{l}\text { Kalongwe, Katanga } \\
\text { Belgian Congo }\end{array}$ & --- & $\begin{array}{l}\text { USGS-XS } \\
\text { USNM-9X9, L }\end{array}$ \\
\hline \multicolumn{2}{|l|}{$\begin{array}{l}\text { Vandenbrandeite } \\
\text { USNM R8389 }\end{array}$} & $\begin{array}{l}\text { Kalongwe, Katanga, } \\
\text { Belglan Congo }\end{array}$ & -- & $\begin{array}{l}\text { USGS-XS } \\
\text { USNM-20 g }\end{array}$ \\
\hline \multicolumn{2}{|l|}{$\begin{array}{l}\text { Vandenbrandeite } \\
\text { USNM R8384 }\end{array}$} & $\begin{array}{l}\text { Kambowe, Katanga, } \\
\text { Belgian Congo }\end{array}$ & --- & $\begin{array}{l}\text { USGS-XS } \\
\text { USNM- } 3 \times 6, M\end{array}$ \\
\hline \multicolumn{2}{|l|}{$\begin{array}{l}\text { Vandenbrandeite } \\
\text { USNM R8391 }\end{array}$} & $\begin{array}{l}\text { Kalongwe, Katanga, } \\
\text { Belgian Congo }\end{array}$ & -- & $\begin{array}{l}\text { USGS-XS } \\
\text { USNM- } 3 \times 9, L\end{array}$ \\
\hline \multicolumn{2}{|l|}{$\begin{array}{l}\text { Vandenbrandeite } \\
\text { USNM } 97250\end{array}$} & $\begin{array}{l}\text { Katanga, Belgian } \\
\text { Congo }\end{array}$ & TE 3144 & --- \\
\hline \multicolumn{2}{|l|}{$\begin{array}{l}\text { Vandenbrandeite } \\
\text { USNM } 97142 \\
\text { Phase } 4\end{array}$} & $\begin{array}{l}\text { Katanga, Belgian } \\
\text { Congo }\end{array}$ & Har. 3772 & USNM-30 g \\
\hline \multicolumn{2}{|l|}{$\begin{array}{l}\text { Vandenbrandeite } \\
\text { USNM } 105125\end{array}$} & $\begin{array}{l}\text { Katanga, Belgian } \\
\text { Congo }\end{array}$ & --- & USNM- $3 \times 3,1$ \\
\hline \multicolumn{5}{|c|}{$\begin{array}{l}\text { I/ Thoreau, J., Soc. géol. Belgique, Annales, } 55 \text { C3 (1933). } \\
2 / \text { Schoep, Alfred, Mus. Congo belge annales I, } 25 \text { (1932). }\end{array}$} \\
\hline \multicolumn{5}{|c|}{ VOGLITE $\mathrm{Ca}_{2} \mathrm{CuU}\left(\mathrm{CO}_{3}\right) \cdot 6 \mathrm{H}_{2} \mathrm{O}$} \\
\hline \multicolumn{5}{|c|}{ Optics } \\
\hline $\begin{array}{l}\text { Orientation } \\
\text { X } \\
\mathrm{Y} \\
\mathrm{Z}\end{array}$ & $\begin{array}{l}\quad n \\
1.541 \\
1.547 \\
1.564\end{array}$ & $\begin{array}{l}\text { Pleochroism } \\
\text { Deep bluish green } \\
\text { Deep bluish green } \\
\text { Pale yellowish }\end{array}$ & $\begin{array}{l}\text { Biaxial psitive } \\
2 v=60^{\circ} \text { (Na) } \\
r<v \text { very stror }\end{array}$ & $(+)$ \\
\hline
\end{tabular}


Voglite (Cont.)

Specimen Data

\begin{tabular}{|c|c|c|c|}
\hline $\begin{array}{l}\text { Minersl and identi- } \\
\text { fying number }\end{array}$ & Location & $\begin{array}{l}\text { Ref. no. of } \\
\text { X-ray patterns }\end{array}$ & Quant1ty \\
\hline $\begin{array}{l}\text { Voglite } \\
\text { USNM C2257 }\end{array}$ & $\begin{array}{l}\text { Joachimsthol, } \\
\text { Bohemia }\end{array}$ & TE 301 & $\begin{array}{l}\text { USGS-XS } \\
\text { USNM-5 } g\end{array}$ \\
\hline
\end{tabular}

WALPURGITE $\mathrm{Bi}_{4}\left(\mathrm{UO}_{2}\right)\left(\mathrm{AsO}_{4}\right)_{2} \mathrm{O}_{4} \cdot 3 \mathrm{H}_{2} \mathrm{O}$ ?

Optics I/

Colorless to pale yellow in transmitted light, not pleochroic. Biaxial negative $(-)$.

$\begin{array}{llc} & n(\text { Joachimsthal) } & n \text { (Schneeberg) } \\ X & 1.90 \pm 0.03 & 1.871 \pm 0.005 \\ \mathrm{Y} & 2.00 \pm 0.03 & 1.975 \pm 0.005 \\ \mathrm{Z} & 2.05 \pm 0.03 & 2.005 \pm 0.005 \\ 2 \mathrm{~V} & \text { Medium large } & 52^{\circ} \text { (calc.) }\end{array}$

Specimen Data

\begin{tabular}{|c|c|c|c|}
\hline $\begin{array}{l}\text { Mineral and identi- } \\
\text { fying number }\end{array}$ & Location & $\begin{array}{l}\text { Ref. no. of } \\
\text { X-ray patterns } \\
\end{array}$ & Quantity \\
\hline $\begin{array}{l}\text { Walpurgite } \\
\text { USNM C4394 }\end{array}$ & $\begin{array}{l}\text { Schneeberg, } \\
\text { Saxony }\end{array}$ & -- & $\begin{array}{l}\text { USGS-XS } \\
\text { USNM- } 3 \times 2, I\end{array}$ \\
\hline $\begin{array}{l}\text { Walpurgite } \\
\text { HMM }\end{array}$ & $\begin{array}{l}\text { Schneeberg, } \\
\text { Saxony }\end{array}$ & $\cdots$ & $\mathrm{HMM}-50 \mathrm{~g}$ \\
\hline $\begin{array}{l}\text { Walpurgite } \\
\text { USNM C4397 }\end{array}$ & $\begin{array}{l}\text { Weisser-Hirsch, } \\
\text { Schneeberg, Saxony }\end{array}$ & -.- & $\begin{array}{l}\text { USGS-XS } \\
\text { USNM- } 3 \times 3, L\end{array}$ \\
\hline $\begin{array}{l}\text { Walpurgite } \\
\text { HMM } 62752\end{array}$ & $\begin{array}{l}\text { Schneeberg, } \\
\text { Saxony }\end{array}$ & Har. 9880 & $\mathrm{HMM}-3 \times 3, I$ \\
\hline $\begin{array}{l}\text { Walpurgite } \\
\text { USNM R5688 }\end{array}$ & $\begin{array}{l}\text { Schneeberg, } \\
\text { Sexony }\end{array}$ & TE 3133 & USNM- $3 \times 3, I$ \\
\hline $\begin{array}{l}\text { Walpurgite } \\
\text { USMM } 4819 \\
\text { Phase } 56\end{array}$ & $\begin{array}{l}\text { Schneeberg, } \\
\text { Sexony }\end{array}$ & Har. 3964 & -- \\
\hline
\end{tabular}

If Larsen, E. S., U. S. Geol. Survey Bull. 679, 155 (1921). 


$$
\begin{gathered}
\text { ZEUNERITE } \mathrm{Cu}\left(\mathrm{UO}_{2}\right)_{2}\left(\mathrm{AsO}_{4}\right)_{4} \cdot 10-16 \mathrm{H}_{2} \mathrm{O} \\
\text { Optics IJ }
\end{gathered}
$$

All specimens of natural so-called zeunerite that have been examined by $X$-ray means have proved to be the meta-I hydrate with 5 to $8 \mathrm{H}_{2} \mathrm{O}$ and to be isostructural with meta-autunite and metatorbernite. Fully hydrated material with 10 to $16 \mathrm{H}_{2} \mathrm{O}$ and isostructural with autunite and torbernite has not yet been shown to occur in nature. In order to maintain parallelism of nomenclature, the name metazeunerite is given here to the species and the name zeunerite proper is held in reserve status for natural occurrence of the 10 to $16 \mathrm{H}_{2} \mathrm{O}$ hydrate that later may be demonstrated.

Artificial Cu( $\left.\mathrm{UO}_{2}\right)_{2}\left(\mathrm{AsO}_{4}\right)_{4} \cdot 10-16 \mathrm{H}_{2} \mathrm{O}$ is tetragonal. Optically uniaxial negative with no $1.602^{4}$ to 1.610 . $^{2}$ The water content varies zeolitically within the range of stability of the phase and the indices of refraction vary therewith. Heated to $65^{\circ} \mathrm{C}$. In air, this phase breaks down to metazeunerite with a water content varying zeolitically between 5 and $8 \mathrm{H}_{2} \mathrm{O}$.

\begin{tabular}{|c|c|c|c|}
\hline $\begin{array}{l}\text { Mineral and identi- } \\
\text { fying number }\end{array}$ & Location & $\begin{array}{l}\text { Ref. no. of } \\
\text { X-ray patterns }\end{array}$ & Quantity \\
\hline $\begin{array}{l}\text { Zeunerite } \\
\text { HMM } 89129\end{array}$ & $\begin{array}{l}\text { Tintic mine, } \\
\text { Eureka, Utah }\end{array}$ & Har. 9793 & $\mathrm{HMM}-\frac{1}{2} \mathrm{~g}$ \\
\hline $\begin{array}{l}\text { Zeunerite } \\
\text { USNM R4385 }\end{array}$ & $\begin{array}{l}\text { Schneeberg, } \\
\text { Saxony }\end{array}$ & -- & $\begin{array}{l}\text { USGS-XS } \\
\text { USNM-6x6, }\end{array}$ \\
\hline $\begin{array}{l}\text { Zeunerite } \\
\text { USNM R5683 }\end{array}$ & $\begin{array}{l}\text { Weisser-Hirsch, } \\
\text { Schneeberg, Saxony }\end{array}$ & --- & $\begin{array}{l}\text { USGS-XS } \\
\text { USNM-6×6, }\end{array}$ \\
\hline $\begin{array}{l}\text { Zeunerite } \\
\text { USNM R6761 }\end{array}$ & $\begin{array}{l}\text { Schneeberg, } \\
\text { Saxony }\end{array}$ & $\mathrm{TE} 3123$ & USNM-6x6,I \\
\hline
\end{tabular}

\section{Specimen Data}

I) Weiss-Frondel, Judith, U. S. Geol. Survey, personal communication.

$$
\begin{gathered}
\text { ZIPPEITE }\left(\mathrm{UO}_{2}\right)_{2}\left(\mathrm{SO}_{4}\right) \mathrm{O} \cdot \mathrm{nH}_{2} \mathrm{O} \\
\text { Optics }
\end{gathered}
$$

Biaxial negative (-), X nearly colorless, $Y$ pale yellow to orange yellow, $Z$ deep yellow to orange yellow. 
Zippeite---Optics (Cont.)

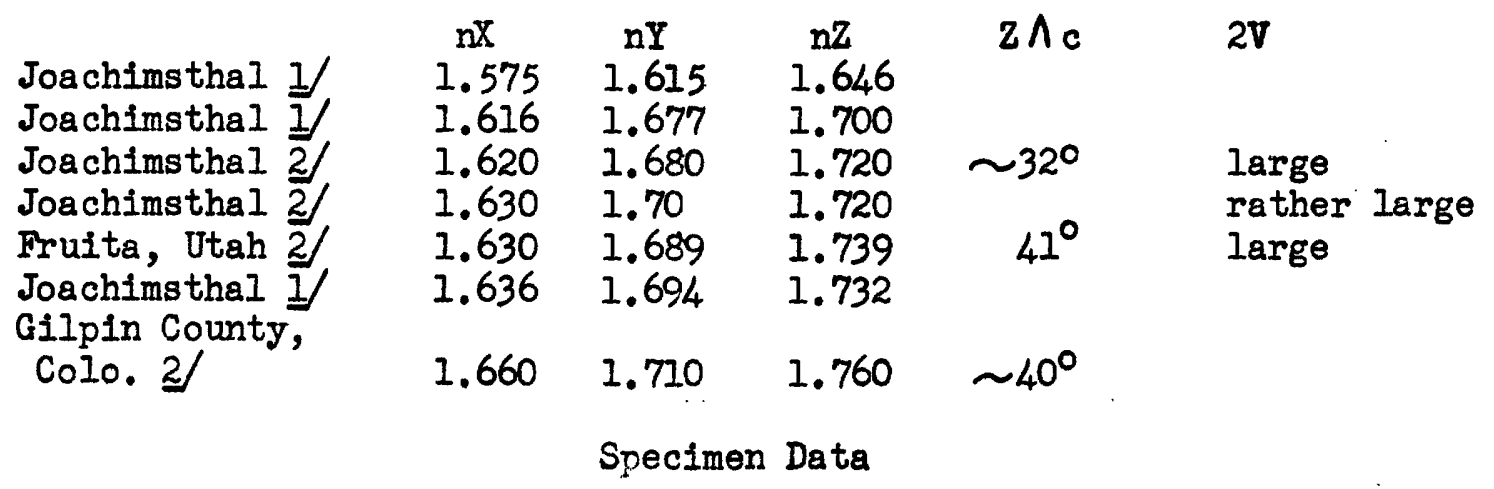

Mineral and identi-
fying number

Zippeite

USNM R7929

Zippeite

USNM C4767

Zippeite

HMM 89612

Phase 64

Mixture?
Location

\section{Joachims thal,}

Bohemia

Joachimsthal,

Bohemia

Joa chims thal,

Bohemia
Ref. no. of

X-ray patterns

Quantity

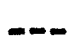

USGS-X

USNM-9 6 , I

TE 251

USGS-S

USNM-3×6,L

If Novácek, R., Soc. royale sci. Bchème, Cl. sci., Méi:. 7 (1935).

Har. 1430

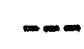

2) Larsen, E. S., U. S. Geol. Survey Bull. 679, 159 (1921). 
GROUP B. MINERRALS WITH MINOR AMOUNTS OF URANTUM AND THORIUU

$$
\text { ALCANITE ( } \mathrm{Ca}, \mathrm{Ce}, \mathrm{Th})_{2}(\mathrm{Al}, \mathrm{Fe}, \mathrm{Mg})_{3} \mathrm{Si}_{3} \mathrm{O}_{12} \text { (OH) }
$$

Specimen Data

\begin{tabular}{|c|c|c|c|}
\hline $\begin{array}{l}\text { Mineral and ident1- } \\
\text { fying number }\end{array}$ & Iocation & $\begin{array}{l}\text { Ref. no. of } \\
\text { X-ray patterns }\end{array}$ & Quantity \\
\hline $\begin{array}{l}\text { Allanite } \\
\text { USMM } 103023\end{array}$ & $\begin{array}{l}\text { Canyon City, } \\
\text { Colo. }\end{array}$ & -- & $\begin{array}{l}\text { USGS-XS } \\
\text { USNM-100 g }\end{array}$ \\
\hline $\begin{array}{l}\text { Allanite } 1 / \\
\text { USNM } 4841 \frac{1}{3}\end{array}$ & $\begin{array}{l}\text { Devil's Head Mountain, } \\
\text { Douglas County, Colo. }\end{array}$ & -- & --- \\
\hline $\begin{array}{l}\text { Allanite } \\
\mathrm{BMM}\end{array}$ & $\begin{array}{l}\text { Blueberry Mountain, } \\
\text { E. Woburn, Mass. }\end{array}$ & Har. 4251 & --- \\
\hline $\begin{array}{l}\text { Allanite } \\
\text { HMM }\end{array}$ & $\begin{array}{l}\text { Rubideaux Mountain } \\
\text { granite, Elsinore quad., } \\
\text { Lalif. }\end{array}$ & Har. 4253 & --- \\
\hline $\begin{array}{l}\text { Allanite } 2 / \\
\text { USNM } 104353\end{array}$ & $\begin{array}{l}\text { Whiteface Mountain, } \\
\text { Essex County, N.Y. }\end{array}$ & -- & USNM-75 g \\
\hline $\begin{array}{l}\text { Allanite } 2 / 1 \\
\text { USNM } 104354\end{array}$ & $\begin{array}{l}\text { Cook Shaft, Mineville, } \\
\text { Essex County, N.Y. }\end{array}$ & --- & $-\cdots$ \\
\hline $\begin{array}{l}\text { Allanite } 3 / \\
\text { USNM } 103601\end{array}$ & $\begin{array}{l}\text { Barringer Hill, } \\
\text { Llano County, Texas }\end{array}$ & $-\cdot-$ & USNM-200 g \\
\hline $\begin{array}{l}\text { Allanite } 4 / \\
\text { USNM } 44456\end{array}$ & Amherst County, Va. & -- & USNM-10 g \\
\hline $\begin{array}{l}\text { Allanite } 5 / \\
\text { USNM } 10355\end{array}$ & $\begin{array}{l}\text { Huddersfield Township, } \\
\text { Quebec }\end{array}$ & -- & USNM-100 g \\
\hline $\begin{array}{l}\text { Allanite } \\
\text { USNM R3834 }\end{array}$ & $\begin{array}{l}\text { Ytterby, } \\
\text { Sweden }\end{array}$ & --- & $\begin{array}{l}\text { USGS-XC } \\
\text { USNM-75 g }\end{array}$ \\
\hline $\begin{array}{l}\text { Allanite } \\
\text { USGS..WTS }\end{array}$ & --- & Inv. 2867 & --- \\
\hline $\begin{array}{l}\text { Allanite } \\
\text { USGS-WTS }\end{array}$ & -- & Inv. 4026 & --- \\
\hline $\begin{array}{l}\text { Allanite } \\
\mathrm{TE}\end{array}$ & --- & $\mathrm{TE} 3220$ & -- \\
\hline $\begin{array}{l}\text { Allanite } \\
\text { TE }(435-W-R S-1)\end{array}$ & $-\cdots$ & TE 528 & --- \\
\hline
\end{tabular}


Allanite---Specimen Data (Cont.)

Yttro-orthite--Synonym of allanite Mineral and identi-
fying number

Yttrium orthite USNM 96847
Location

North Karelia, Russia
Ref. no. of X-ray patterns

Quantity

USGS-XS

USNM-150 g

If Eakins, I. G., Proc. Colo. Sci. Soc. 2, 32 (1886).

2) Marble, J. P., Am. Jour. Sci., 5th ser., 241, 32-42 (1943).

3/ Marble, J. P., Am. Mineralogist, 25, 168-173 (1940).

4 Marble, J. P., Am. Jour. Sci., 5th ser., 30, 349-352 (1935).

5) Marble, J. P., Nat. Research Council Rept. Comm. Measurement Geol. Time, 1942.

\section{BASTNAESITE $(\mathrm{Ce}, \mathrm{Ia})\left(\mathrm{CO}_{3}\right) \mathrm{F}$}

Optics I/

In transmitted light, colorless to pale yellow. Faintly pleochroic with absorption $\mathrm{E}>0$.

$\begin{array}{cccc} & n \text { (Bastnaes) } & n(\text { P1kes Peak) } & \text { Uniaxial positive (t) } \\ 0 & 1.722 & 1.717 & \\ \mathrm{E} & 1.823 & 1.818 & \end{array}$

Specimen Data

Mineral and identi- Location fying number

Bastneesite TE

Bastnaesite USNM R7792

Bastnaesite HMM

Bastnaesite 2/ USNM 48415

Bastnaesite 2/ USMM 81857

Bastnaesite I/ USNM 105628-631 Calif.
Birthday prospect, San Bernardino County,

Stove Mountain, near

Pikes Peak, Colo.

Ref. no. of X-ray patterns

Stove Mountain, St. Peter's dome, Colo.

St. Peter's dome, Pikes Peak region, Colo.

Cheyenne Mountain, Pikes

Peak region, Colo.

Red Cloud mine, Gallinas -.Mountains, Lincoln

County, N. Mex.
TE 255

USGS-XSC

USNM-15 g

Quantity

USGS-XS

USNM-25 g

Har. 9706

$-\cdot$

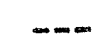

USNM-200 g 
Bastnaesite---Specimen Data (Cont.)

\begin{tabular}{|c|c|c|c|}
\hline $\begin{array}{l}\text { Mineral and identi- } \\
\text { fying number }\end{array}$ & Location & $\begin{array}{l}\text { Ref. no, of } \\
\text { X-ray patterns }\end{array}$ & Quantity \\
\hline $\begin{array}{l}\text { Bastnaesite I/ } \\
\text { USNM R2617 }\end{array}$ & Madagascar & Inv. 1512 & \\
\hline
\end{tabular}

I/ Glass, J. J., and Smalley, R. G., Am. Mineralogist 30, 601-615 (1945).

2/ Hillebrand, W. F., Am. Jour. Sci., 4th ser., 7, 51 (1899).

CAPPELENITE $(\mathrm{Ba}, \mathrm{Y})_{2} \mathrm{SiBO}_{6}$

Specimen Data

\begin{tabular}{|c|c|c|c|}
\hline $\begin{array}{l}\text { Mineral and identi- } \\
\text { fying number } \\
\end{array}$ & Location & $\begin{array}{l}\text { Ref. no. of } \\
\text { x-ray patterns }\end{array}$ & Quantity \\
\hline $\begin{array}{l}\text { Cappelenite } \\
\text { HMM } 85281\end{array}$ & $\begin{array}{l}\text { Langesund Fiord, } \\
\text { Norway }\end{array}$ & $\begin{array}{l}\text { Har. } 11319 \\
\text { Not ignited } \\
\text { Very faint patt }\end{array}$ & ern \\
\hline $\begin{array}{l}\text { Cappelenite } \\
\text { HMM } 85281\end{array}$ & $\begin{array}{l}\text { Langesund Fiord, } \\
\text { Norway }\end{array}$ & $\begin{array}{l}\text { Har. } 11351 \\
\text { Ignited } \\
\text { Same pattern as } \\
\text { un-ignited but }\end{array}$ & stronger \\
\hline
\end{tabular}

\section{CERITE $\mathrm{Ce}_{2} \mathrm{SiO}_{5}$ \\ Specimen Data}

\section{Mineral and identi- fying number}

Cerite

TE

Carite

USNM 45326

Cerite

USNM R7073

Cerite

HMM
Location

Jamestown district, Colo.

Bastnaes,

Sweden

Bastnaes,

Sweden

Bastnaes, Sweden
Ref. no. of Quantity X-ray patterns

USGS-XSC

USGS-XS

USNM- $100 \mathrm{~g}$

TE 635

USGS-XS

USNM-500 g

Har. 7957

HMAM $-500 \mathrm{~g}$ 
CORVUSITE $\mathrm{V}_{2} \mathrm{O}_{4} \cdot 6 \mathrm{~V}_{2} \mathrm{O}_{5} \cdot \times \mathrm{H}_{2} \mathrm{O}$ ?

Specimen Data

\begin{tabular}{|c|c|c|c|}
\hline $\begin{array}{l}\text { Mineral and identi- } \\
\text { fying number }\end{array}$ & Location & $\begin{array}{l}\text { Ref. no. of } \\
X \text {-ray patterns }\end{array}$ & Quantity \\
\hline $\begin{array}{l}\text { Corvusite } \\
\text { USNM } 102627\end{array}$ & $\begin{array}{l}\text { Gateway, } \\
\text { Colo. }\end{array}$ & --- & $\begin{array}{l}\text { USGS-XS } \\
\text { USNM-75 g }\end{array}$ \\
\hline $\begin{array}{l}\text { Corvusite } \\
\text { TE }\end{array}$ & $\begin{array}{l}\text { Small Spot mine, } \\
\text { Mesa County, Colo. }\end{array}$ & TE 503 & USGS-XS \\
\hline $\begin{array}{l}\text { Corvusite } \\
\text { TE-LRS/58/48 }\end{array}$ & $\begin{array}{l}\text { Club mine, Montrose } \\
\text { County, Colo. }\end{array}$ & TE 464 & --- \\
\hline $\begin{array}{l}\text { Corvusite I/ } \\
\text { USNM } 96806\end{array}$ & $\begin{array}{l}\text { Tomlinson and Hart basin } \\
\text { claims, } 9 \text { mi. W. of } \\
\text { Gateray, Colo. }\end{array}$ & $\mathrm{TE} 472$ & USNM-100 g \\
\hline $\begin{array}{l}\text { Corvusite I/ } \\
\text { USM } 96807\end{array}$ & $\begin{array}{l}\text { Ponto No. } 3 \text { claim, N. wa } \\
\text { Gypsum Valley, San Migue } \\
\text { County, Colo. }\end{array}$ & 11 & USMM-4000 \\
\hline $\begin{array}{l}\text { If Henderson, E. } \\
(1933) .\end{array}$ & P., and Hess, F. I., Am. & Mineralogist 18 , & , $195-205$ \\
\hline DAVIDITE - C & $\begin{array}{c}\text { ontains } \mathrm{Ti}, \mathrm{Fe} \text {, rare earth } \\
\text { Specimen Data }\end{array}$ & $s, U, V$, and $\mathrm{Cr}$. & \\
\hline $\begin{array}{l}\text { Mineral and identi- } \\
\text { fying number }\end{array}$ & Location & $\begin{array}{l}\text { Ref. no. of } \\
\text { X-ray patterns }\end{array}$ & Quantity \\
\hline $\begin{array}{l}\text { Davidite } \\
\text { USNM 87282 }\end{array}$ & $\begin{array}{l}\text { Olary, Scuth } \\
\text { Australia }\end{array}$ & $\mathrm{TE} 605$ & $\begin{array}{l}\text { USGS-XS } \\
\text { USNM-150 g }\end{array}$ \\
\hline
\end{tabular}

$$
\begin{gathered}
\text { HIELMITE } \mathrm{AB}_{2} \mathrm{O}_{6} \text { or } \mathrm{A}_{2} \mathrm{~B}_{3} \mathrm{O}_{10} \\
\mathrm{~A}=\mathrm{Y}, \mathrm{Fe}^{2}, \mathrm{U}^{4}, \mathrm{Mn}, \mathrm{Ca} ; \mathrm{B}=\mathrm{Cb}, \mathrm{Ta}, \mathrm{Sn}, \mathrm{W} \\
\text { Optics } \mathrm{I}
\end{gathered}
$$

In transmitted light, dark yellowish brown to opaque. Anisotropic, but some has been altered externally or along fractures to an isotropic material. Optically positive $(t)$, and nearly or quite uniaxial. 
Hielmite---Optics (Cont.)

$\begin{array}{lll} & { }^{n_{L i}} & \text { Pleochroism } \\ X & 2.30 \pm 0.02 & \text { Yellowish brown } \\ Z & 2.40 \pm 0.04 & \text { Nearly opaque }\end{array}$

Specimen Data

\begin{tabular}{|c|c|c|c|}
\hline $\begin{array}{l}\text { Mineral and identi- } \\
\text { fying number }\end{array}$ & Location & $\begin{array}{l}\text { Ref. no. of } \\
\text { X-ray patterns }\end{array}$ & Quantity \\
\hline $\begin{array}{l}\text { Hielmite } \\
\text { HMM } 57842\end{array}$ & $\begin{array}{l}\text { Kårarfvet, } \\
\text { near Fahlun, Sweden }\end{array}$ & $\begin{array}{l}\text { Har: } 1913 \\
\text { Ignited }\end{array}$ & $-\cdots-$ \\
\hline $\begin{array}{l}\text { Hielmite } \\
\text { USNM C4006 }\end{array}$ & $\begin{array}{l}\text { Kårarfvet, } \\
\text { Sweden }\end{array}$ & TE 633 & --- \\
\hline $\begin{array}{l}\text { Hielmite } \\
\text { HMM } 85106\end{array}$ & $\begin{array}{l}\text { Striposem, } \\
\text { Sweden }\end{array}$ & $\begin{array}{l}\text { Har. } 1906 \\
\text { Ignited - } \\
\text { same as fergusc } \\
\text { Petaca, N. Mex. }\end{array}$ & ite, \\
\hline
\end{tabular}

I) Larsen, E. S., U. S. Geol. Survey Bul1. 679, 85 (1921).

HOKUTOLITE - Variety of barite

Specimen Data

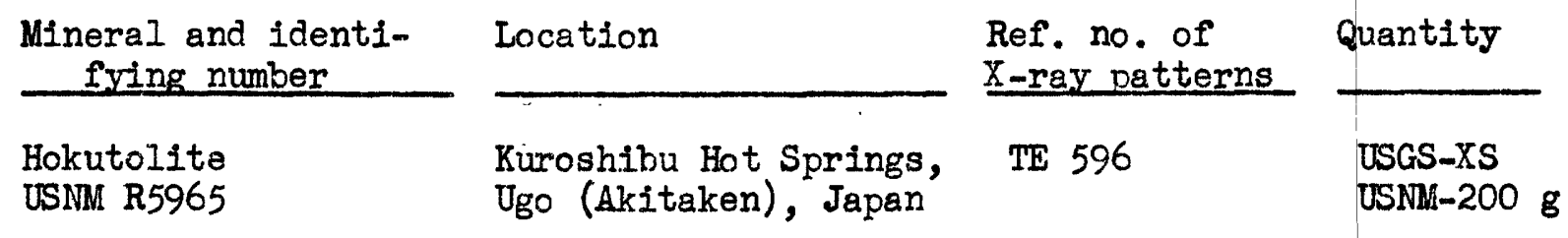

JOHNSTRUPITE - A complex silicate of $\mathrm{Na}, \mathrm{Ca}, \mathrm{Th}, \mathrm{C} \theta$, and $\mathrm{Ti}$

Specimen Data

$\begin{array}{llcl}\begin{array}{l}\text { Mineral and identi- Location } \\ \text { fying number }\end{array} & \text { Brevig, Norway } & \begin{array}{l}\text { Ref. no. of } \\ \text { X-ray patterns }\end{array} & \text { Quantity } \\ \begin{array}{l}\text { Johnstrupite } \\ \text { USNM R5016 }\end{array} & \ldots & \ldots \text { USGS-X } \\ \text { USMM-5 g }\end{array}$ 
KOLM - Rock with hydrocarbon and uranium

Specimen Data

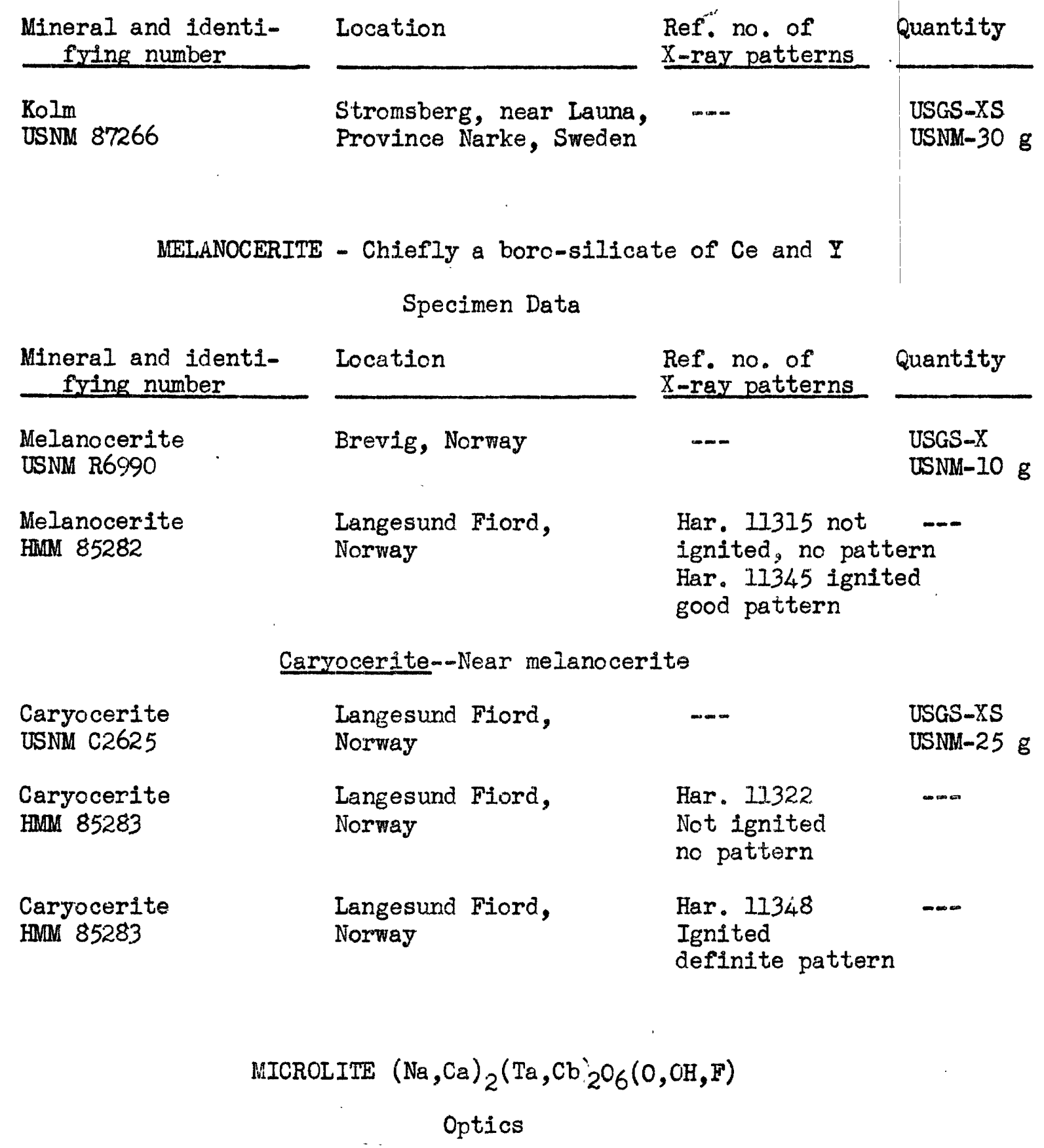

Isotropic, but may show weak anomalous birefringence in the nonmetamict material. Colorless, or pale yellow, brown, etc., in material with a deep body color; sometimes cloudy. May show a zonal structure 
Microlite---Optics (Cont.)

in shades of brown or yellow. $n$ apparently decreases with increasing alteration by hydration. On ignition $n$ increases to $2.0-2.2 .1$ J

\section{Specimen Data}

\begin{tabular}{|c|c|c|c|}
\hline $\begin{array}{l}\text { Mineral and identi- } \\
\text { fying number }\end{array}$ & Location & $\begin{array}{l}\text { Ref. no. of } \\
\text { X-ray patterns }\end{array}$ & Quantity \\
\hline $\begin{array}{l}\text { Microlite } \\
\text { USNM } 104745\end{array}$ & $\begin{array}{l}\text { Brown Derby mine, } \\
\text { Mentrose County, Colo. }\end{array}$ & -- & $\begin{array}{l}\text { USGS-XS } \\
\text { USNM-20 g }\end{array}$ \\
\hline $\begin{array}{l}\text { Microlite } \\
\text { HMM }\end{array}$ & $\begin{array}{l}\text { Topsham, } \\
\text { Maine }\end{array}$ & Har. 453 & -- \\
\hline $\begin{array}{l}\text { Microlite } 2 / \\
\text { USNM } 100282\end{array}$ & $\begin{array}{l}\text { Moorefield mine, } \\
\text { Amelia, Va. }\end{array}$ & --- & USNM-10 g \\
\hline $\begin{array}{l}\text { Microlite } 2 / \\
\text { USMM } 100282\end{array}$ & $\begin{array}{l}\text { Rutherford mine, } \\
\text { Amelia, Va. }\end{array}$ & -- & USNM-10 $\mathrm{g}$ \\
\hline $\begin{array}{l}\text { Microlite } \\
\text { USNM } 93594\end{array}$ & Amelia, Va. & --- & $\begin{array}{l}\text { USGS-XS } \\
\text { USNM-10 g }\end{array}$ \\
\hline Microlite & -- & Inv. 2825 & --- \\
\hline \multicolumn{4}{|c|}{ Calciotantalite--Possibly a mixture of microlite and tantalite } \\
\hline \multirow[t]{2}{*}{$\begin{array}{l}\text { Calciotantalite } \\
\text { USNM R6520 }\end{array}$} & $\begin{array}{l}\text { Pilbara Gold district, } \\
\text { Western Australia }\end{array}$ & -- & $\begin{array}{l}\text { USGS-XS } \\
\text { USNM-30 g }\end{array}$ \\
\hline & $\begin{array}{c}\text { Iite--An altered microli } \\
\text { composition close }\end{array}$ & $\begin{array}{l}\text { te with } \\
\text { to tantalite }\end{array}$ & \\
\hline $\begin{array}{l}\text { Neotantalite } \\
\text { USNM R5095 }\end{array}$ & $\begin{array}{l}\text { Department Allier, } \\
\text { France }\end{array}$ & $\begin{array}{l}\text { TE } 746 \\
\text { Ignited }\end{array}$ & $\begin{array}{l}\text { USGS-X } \\
\text { USNM-200 g }\end{array}$ \\
\hline Neotantalite & $\begin{array}{l}\text { Department Allier, } \\
\text { France }\end{array}$ & Har. 231 & --- \\
\hline
\end{tabular}


MOSANDRITE - Complex silicate of $\mathrm{Na}, \mathrm{Ca}, \mathrm{Ce}$, and $\mathrm{TI}$

Specimen Data

\begin{tabular}{|c|c|c|c|}
\hline $\begin{array}{l}\text { Mineral and identi- } \\
\text { fying number }\end{array}$ & Location & $\begin{array}{l}\text { Ref. no. of } \\
\text { X-roy patterns }\end{array}$ & Quantity \\
\hline $\begin{array}{l}\text { Mosandrite } \\
\text { USMM R7136 }\end{array}$ & $\begin{array}{l}\text { Brevig, } \\
\text { Norway }\end{array}$ & --- & $\begin{array}{l}\text { USGS-XS } \\
\text { USNM-10 }\end{array}$ \\
\hline
\end{tabular}

\footnotetext{
PISEKITE - Essentially a columbate-tantalate-titanate of $U$ and rare earths, with $T h$ and Sn.
}

Specimen Data

\begin{tabular}{|c|c|c|c|}
\hline $\begin{array}{l}\text { Mineral and identi- } \\
\text { fying number }\end{array}$ & Location & $\begin{array}{l}\text { Ref. no. of } \\
\text { X-ray patterns }\end{array}$ & Quantity \\
\hline $\begin{array}{l}\text { Pisekite? } \\
\text { USNM R7434 }\end{array}$ & $\begin{array}{l}\text { Pisek, } \\
\text { Dohemia }\end{array}$ & -- & $\begin{array}{l}\text { USGS-X } \\
\text { USNM-2 g }\end{array}$ \\
\hline
\end{tabular}

\author{
PYROCHLORE $(\mathrm{Na}, \mathrm{Ca})_{2}(\mathrm{Cb}, \mathrm{Ta})_{2} \mathrm{O}_{6} \mathrm{~F}$ \\ Optics
}

Isotropic, but may show weak anomalous birefringence in the nonmetamict material. Colorless, or pale yellow, brown, etc., in material with a deep body color; some may be cloudy. May show a zonal structure in shades of brown or yellow. $n$ apparently decreases with increasing alteration by hydration. On ignition n increases to 2.0 - 2.2.1/ Pyrrhite has $n=2.162 /$ and koppite has $n=2.12$ to 2.18 .

Specimen Data

\begin{tabular}{|c|c|c|c|}
\hline $\begin{array}{l}\text { Mineral and identi- } \\
\text { fying number }\end{array}$ & Location & $\begin{array}{l}\text { Ref. no. of } \\
\text { X-ray patterns }\end{array}$ & Quantity \\
\hline $\begin{array}{l}\text { Pyrochlore } \\
\text { USNM R5033 }\end{array}$ & $\begin{array}{l}\text { Fredricksvärn, } \\
\text { Norway }\end{array}$ & $\begin{array}{l}\text { T: } 737 \\
\text { Ignited }\end{array}$ & $\begin{array}{l}\text { USGS-XS } \\
\text { USNM-30 g }\end{array}$ \\
\hline $\begin{array}{l}\text { Pyrochlore } \\
\text { HMM } 87780\end{array}$ & $\begin{array}{l}\text { Miask, } \\
\text { Urals }\end{array}$ & $\begin{array}{l}\text { Har. } 2039 \\
\text { Ignited }\end{array}$ & $\begin{array}{l}\text { USGS-XS } \\
\text { HMM-20 g }\end{array}$ \\
\hline
\end{tabular}


Pyrochlore----Specimen Data (Cont.)

Chalcolamprite--Synonym of pyrochlore

\begin{tabular}{|c|c|c|c|}
\hline $\begin{array}{l}\text { Mineral and identi- } \\
\text { fying number }\end{array}$ & Location & $\begin{array}{l}\text { Ref. no. of } \\
\text { X-ray patterns }\end{array}$ & Quantity \\
\hline $\begin{array}{l}\text { Chalcolamprite } \\
\text { HMM } 87784\end{array}$ & $\begin{array}{l}\text { Narasuk, } \\
\text { Greenland }\end{array}$ & Har. 434 & HMM- $5 \mathrm{~g}$ \\
\hline \multicolumn{4}{|c|}{$\begin{array}{r}\text { Ellsworthite--Composition is close to prrochlore, } \\
\text { but analyses show it is relatively } \\
\text { high in } \mathrm{U} \text { and } \mathrm{H}_{2} \mathrm{O} \text { and low in alkalies }\end{array}$} \\
\hline $\begin{array}{l}\text { Ellsworthite } \\
\text { USNM } 94804\end{array}$ & Ontario & -- & $\begin{array}{l}\text { USGS-XS } \\
\text { USNM-2O }\end{array}$ \\
\hline Ellsworthite & $\begin{array}{l}\text { Hastings County, } \\
\text { Ontario }\end{array}$ & $\begin{array}{l}\text { Inv . } 2997 \\
\text { Ignited }\end{array}$ & $\cdots$ \\
\hline $\begin{array}{l}\text { Ellsworthite } \\
\text { FMM } 81175\end{array}$ & Hybia, Ontario & $\begin{array}{l}\text { Har. } 2035 \\
\text { Ignited }\end{array}$ & -- \\
\hline $\begin{array}{l}\text { Ellsworthite } \\
\text { HMM } 90525\end{array}$ & $\begin{array}{l}\text { Haliburton County, } \\
\text { Ontario }\end{array}$ & $\begin{array}{l}\text { Har. } 333 \\
\text { Ignited }\end{array}$ &.-- \\
\hline $\begin{array}{l}\text { EIlsworthite } \\
\text { HMM } 89636\end{array}$ & $\begin{array}{l}\text { Haliburton County, } \\
\text { Ontario }\end{array}$ & $\begin{array}{l}\text { Har. } 433 \\
\text { Ignited }\end{array}$ & -- \\
\hline $\begin{array}{l}\text { ElIsworthite } \\
\text { HMM } \$ 9636\end{array}$ & $\begin{array}{l}\text { Haliburton County, } \\
\text { Ontario }\end{array}$ & $\begin{array}{l}\text { Har. } 469 \\
\text { No pattern }\end{array}$ & --- \\
\hline
\end{tabular}

Endelolite--Similar in composition to chalcolamprite

Endeiolite

USNM C4018
Nersarsuk,

Greenland
USGS-XS USNM-5 $\mathrm{g}$

Hatchettolite--Uranjan pyrochlore

Hatchettolite

HMM 87786

Hatchettolite

HMM 91477

Hatchettolite

USNM 97640
Mitchell County, N.C. Har. 2036

Ignited

Hybla,

Ontario

Har. 2038

Ignited

San Josê de Bujaube

Minas Geraes, Brazil
TE 638
HMN-100 g 
Pyrochlore---Specimen Data (Cont.)

Koppite--Synonym of pyrochlore

\begin{tabular}{|c|c|c|c|}
\hline $\begin{array}{l}\text { Mineral and identi- } \\
\text { fying number }\end{array}$ & Iocation & $\begin{array}{l}\text { Ref. no, of } \\
\text { X-ray patterns }\end{array}$ & Quantity \\
\hline Koppite & $\begin{array}{l}\text { Near Schelingen, } \\
\text { Kalserstuhl, Germany }\end{array}$ & $-\infty$ & USGS-XS \\
\hline & nacite--Synonym of $\mathrm{py}$ & hlore & \\
\hline $\begin{array}{l}\text { Marignacite } \\
\text { USNM R5037 }\end{array}$ & Wausau, Wis. & TE 656 & 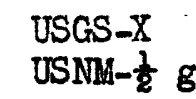 \\
\hline $\begin{array}{l}\text { Marignacite } \\
\text { HMM } 87782\end{array}$ & Wausau, Wis. & $\begin{array}{l}\text { Har. } 230 \\
\text { Ignited } \\
\text { Har. 1191-not } \\
\text { ignited, poor } \\
\text { pattern }\end{array}$ & -- \\
\hline
\end{tabular}

Pyrrhite--Synonym of pyrochlore

Pyrrhite?

Azores

USGS-X

USNM-1 $g$

USNM C3963

If Larsen, E. S., and Berman, Harry, U. S. Geol. Survey Bull. 848, 60,61 (1934).

2) Larsen, E. S., U. S. Geol. Survey Bull. 679, 124, 180 (1921). Glass, J. J., Am. Mineralogist 20, 752 (1935).

Kreutz, Stefan, Akad. Wiss., Krakau, 54, 227 (1915).

Kreutz, Stefan, Jahrb. Min. 134 (1918).

$$
\text { ROWLANDITE - An yttrium silicate }
$$

Specimen Data

$\begin{array}{llcl}\begin{array}{l}\text { Mineral and identi- } \\ \text { fying number }\end{array} & \text { Location } & \begin{array}{c}\text { Ref. no. of } \\ \text { X-ray patterns }\end{array} & \text { Quantity } \\ \begin{array}{l}\text { Rowlanaite } \\ \text { USNM R3784 }\end{array} & \begin{array}{l}\text { Llano County, } \\ \text { Texas }\end{array} & -\cdots & \text { USGS-X }\end{array}$




$$
\begin{aligned}
& \text { STEENSTRUPINE - Complex silicate of rare earths, } \\
& \text { Th, Na, } \mathrm{K}, \mathrm{IO}, \mathrm{Iin}, \mathrm{Mg}, \mathrm{P}, \mathrm{BO}, \mathrm{Al} \text {, and } \mathrm{Ta} \\
& \text { w1th }(\mathrm{OH}) \text { and } \mathrm{F} \text {. }
\end{aligned}
$$

\begin{tabular}{|c|c|c|c|}
\hline $\begin{array}{l}\text { Mineral and ident1- } \\
\text { fying number }\end{array}$ & Iocation & $\begin{array}{l}\text { Ref. no. of } \\
X-\text { ray patterns }\end{array}$ & Quantity \\
\hline $\begin{array}{l}\text { Steenstrupine } \\
\text { USNM R3317 }\end{array}$ & $\begin{array}{l}\text { Kangerdluarsuk, } \\
\text { Greenland }\end{array}$ & -- & $\begin{array}{l}\text { USGS-XS } \\
\text { USNM-30 } \mathrm{g}\end{array}$ \\
\hline
\end{tabular}

\section{Specimen Data}

$$
\begin{array}{ll}
\text { TENGERITE } & \text { a) Close to } \mathrm{CaY}_{3}(\mathrm{OH})_{3}\left(\mathrm{CO}_{3}\right)_{4} \cdot 3 \mathrm{H}_{2} \mathrm{O} \\
\text { b) Supposediy an yttrium carbonate }
\end{array}
$$

$$
\text { Optics }
$$

Material from Itterby, supposedly tengerite, was optically positive $(t)$

\begin{tabular}{|c|c|c|c|}
\hline $\begin{array}{l}\text { Mineral and identi- } \\
\text { fying number }\end{array}$ & Location & $\begin{array}{l}\text { Ref. no. of } \\
X \text {-ray patterns }\end{array}$ & Quantity \\
\hline $\begin{array}{l}\text { Tengerite } \\
\text { OSNM } 104044\end{array}$ & $\begin{array}{l}\text { Iisaka, Fukushima, } \\
\text { Japan }\end{array}$ & -- & $\begin{array}{l}\text { USCS-XS } \\
\text { USNM-10 g }\end{array}$ \\
\hline
\end{tabular}
with $2 \mathrm{~V}$ large, $\mathrm{nX}=1.555, \mathrm{nY}=1.585$, and $\mathrm{nX}$ parallel to the elongation. I Alteration product at Iisaka, Japan, also classed with tengerite, occurred as a white powder or as globular concretions with a minute scaly structure and pearly luster. $n X=1.622, n Y-1.642$.

\begin{tabular}{|c|c|c|c|}
\hline $\begin{array}{l}\text { Mineral and identi- } \\
\text { fying number }\end{array}$ & Location & $\begin{array}{l}\text { Ref. no. of } \\
\text { X-ray patterns }\end{array}$ & Quantity \\
\hline $\begin{array}{l}\text { Thalenite } \\
\text { USNM R3782 }\end{array}$ & $\begin{array}{l}\text { Osterby, Dalecarlia, } \\
\text { Sweden }\end{array}$ & -- & $\begin{array}{l}\text { USGS-XS } \\
\text { USNM-30 }\end{array}$ \\
\hline $\begin{array}{l}\text { Thalenite } \\
\text { Han } 85996\end{array}$ & $\begin{array}{l}\text { Torsekebacken, } \\
\text { Sweden }\end{array}$ & $\begin{array}{l}\text { Har. } 7962 \text { not } \\
\text { ignited; pattern } \\
\text { only one line - } \\
\text { metamict? }\end{array}$ & $\cdots$ \\
\hline
\end{tabular}

\section{Specimen Data}

I/ Larsen, E. S., U. S. Geol. Survey Bull. 679, 1/42 (1921).

$$
\text { THALENITE } \mathrm{I}_{4} \mathrm{Si}_{4} \mathrm{O}_{13}(\mathrm{OH})_{2}
$$

Specimen Data 
THUCOLITE - A hydrocarbon

Specimen Data

\begin{tabular}{|c|c|c|c|}
\hline $\begin{array}{l}\text { Mineral and identi- } \\
\text { fying number }\end{array}$ & Location & $\begin{array}{l}\text { Ref. no. of } \\
\text { X-ray patterns }\end{array}$ & Quantity \\
\hline $\begin{array}{l}\text { Thucolite } \\
\text { USNM } 103138\end{array}$ & $\begin{array}{l}\text { Besner mine, Henvey } \\
\text { Township, Parry Sound, } \\
\text { Ontario }\end{array}$ & --- & USGS-XS \\
\hline TRITONITE - & $\begin{array}{c}\text { A boro-silicate of ceri } \\
\text { calcium, and thorium, } \\
\text { Ing fluorine. Exact fo } \\
\text { Specimen Data }\end{array}$ & $\begin{array}{l}\text { um, yttrium, } \\
\text { lso contain- } \\
\text { rmula uncertain. }\end{array}$ & \\
\hline $\begin{array}{l}\text { Mineral and identi- } \\
\text { fying number }\end{array}$ & Location & $\begin{array}{l}\text { Ref. no. of } \\
\text { x-ray patterns }\end{array}$ & Quantity \\
\hline $\begin{array}{l}\text { Tritomite } \\
\text { USNM R3319 }\end{array}$ & Brevig, Norway & -- & $\begin{array}{l}\text { USGS-X } \\
\text { USNM-5 g }\end{array}$ \\
\hline $\begin{array}{l}\text { Tritomite } \\
\text { HMM } 85290\end{array}$ & $\begin{array}{l}\text { Langesund Fiord, } \\
\text { Norway }\end{array}$ & $\begin{array}{l}\text { Har. } 11320 \\
\text { Not ignited, } \\
\text { no pattern } \\
\text { Har. } 11349 \\
\text { Ignited, good } \\
\text { pattern }\end{array}$ & $\cdots$ \\
\hline
\end{tabular}

TSCHEFFKINITE - Complex silicate of rare earths, $\mathrm{Fe}, \mathrm{Mn}, \mathrm{Mg}, \mathrm{Ca}, \mathrm{AI}, \mathrm{TI}, \mathrm{Th}$, and $\mathrm{U}$

Specimen Data

\begin{tabular}{|c|c|c|}
\hline $\begin{array}{l}\text { Mineral and identi- } \\
\text { fying number }\end{array}$ & Location & $\begin{array}{l}\text { Ref. no. of Quantity } \\
\text { X-ray patterns }\end{array}$ \\
\hline $\begin{array}{l}\text { Tscheffikinite } \\
\text { HMM } 85151\end{array}$ & $\begin{array}{l}\text { Fiadanana, } \\
\text { Madagascar }\end{array}$ & $\begin{array}{l}\text { Har. } 11318 \text { not } \\
\text { ignited, no pattern } \\
\text { Har. } 11347 \text { ignited, } \\
\text { good pattern }\end{array}$ \\
\hline $\begin{array}{l}\text { Tscheffkinite } \\
\text { HMM } 96869\end{array}$ & $\begin{array}{l}\text { Ilmen Mountains, } \\
\text { Urals }\end{array}$ & $\begin{array}{l}\text { Har. } 11317 \text { not } \\
\text { ignited, no pattern } \\
\text { Har. } 11344 \text { ignited, } \\
\text { good pattern but } \\
\text { not same as HMM } 85151\end{array}$ \\
\hline
\end{tabular}


Tscheffkinite---Specimen Data (Cont.)

Chevkinite $=$ tscheffkinite

Mineral and identi-
fying number

Chevkinite I/

USNM 105344
Location

Aquarious Mountains, Mohave County, Ariz.
Ref. no. of Quantity X-ray patterns

USNM-75 $\mathrm{g}$

1) Kauffman, A. J., and Jaffe, H. W., Am. Mineralogist, 31, 582-588 (1946).

$$
\begin{aligned}
\text { VANOXITE - } & \text { A hydrated vanadium oxide, perhaps } \\
& 2 \mathrm{~V}_{2} \mathrm{O}_{4} \cdot \mathrm{V}_{2} \mathrm{O}_{5} \cdot 8 \mathrm{H}_{2} \mathrm{O} \text {, reported to contain } \\
& \text { up to } 0.5 \% \mathrm{~J} \text {. }
\end{aligned}
$$

Specimen Data

\author{
Mineral and Identi- Location \\ Ref. no. of \\ fying number \\ X-ray patterns \\ Quantity \\ Vanoxite I/ \\ USNM 95059 \\ Bill Bryan claim, \\ Wild Steer canyon, \\ TE 474- \\ USGS-S \\ Montrose County, Colo. picture \\ If Hess, F. I., U. S. Geol. Survey Bull. 750-D, 63 (1925).

$$
\begin{gathered}
\text { VOLBORTHITE } \mathrm{Cu}_{2}\left(\mathrm{VO}_{4}\right)(\mathrm{OH}) \cdot \mathrm{H}_{2} \mathrm{O}(?) \\
\text { Optics }
\end{gathered}
$$
Biaxial.

In transmitted light, green to greenish yellow. Faintly pleochroic.

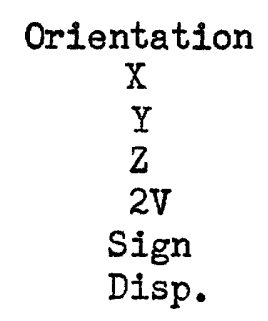

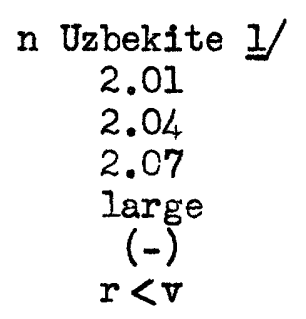
n Utah $2 /$ 2.01 2.05 2.10 (caic.) $68^{\circ}(\mathrm{Li}) 83^{\circ}(\mathrm{Na})$ $(+)$ red, (-) violet $r>\nabla$ (inclined)


Volborthite (Cont.)

\section{Specimen Data}

\begin{tabular}{|c|c|c|c|}
\hline $\begin{array}{l}\text { Mineral and identi- } \\
\text { fying number }\end{array}$ & Location & $\begin{array}{l}\text { Ref. no. of } \\
\text { X-ray patterns }\end{array}$ & Quantity \\
\hline $\begin{array}{l}\text { Volborthite } \\
\text { USGS(wTS } 49-45 \operatorname{CSX} \text { ) } \\
\text { Type specimen }\end{array}$ & Russia & Inv. 4463 & --- \\
\hline
\end{tabular}

$$
\text { Calciovolborthite - } \mathrm{Cu}, \mathrm{Ca}\left(\mathrm{VO}_{4}\right)(\mathrm{OH})(?)
$$

Calciovolborthite $2 /$ USNM 93298

\section{Richardson, Grand} River, Utah

USNRL-4x10,M
1/ Barth, T. F. W., and Berman, Harry, Chemie der Erde, 5, 30 (1930). 2/ Hillebrand, W. F., and Merwin, H. E., Am. Jour. Sci., 4th ser., 35, 441 (1913) on material termed calciovolborthite (?).
WIIKITE - Ill-defined mixture and alteration product of minerals high in $\mathrm{Cb}, \mathrm{Ta}, \mathrm{Ti}, \mathrm{Si}$, and $\mathrm{Y}$.

Specimen Data
Wiikite
USNM R5119
Mineral and identi- fying number
Location
Ref. no. of
$X-$ ray patterns
Quantity
Wiikite
HMM 58172
Lokansuari, Impilahti, Finland
USGS-XS
USNM-100 g
Har. 11325 not
ignited, no pattern
Har. 11316 not
ignited, faint pattern
Har. 11330 ignited, good
pattern but not same pattern as un-ignited material

\section{XENOTIME $\mathrm{Y}\left(\mathrm{PO}_{4}\right)$}

\section{Optics I/}

In transmitted light, colorless to very pale yellowish green, yellow or yellowish brown. 
Xenotime---Optics (Cont.)

$\begin{array}{llll} & \mathrm{n} \mathrm{Na} & \mathrm{n} \mathrm{Na} \text { (New Zealand) } & \\ 0 & 1.721 & 1.720 & \text { Uniaxial positive (t) } \\ \mathrm{E} & 1.816 & 1.827 & \end{array}$

Xenotime may be weakly dichroic with 0 pink, yellow, or yellowish brown; and E brownish yellow, grayish brown, or greenish.

Specimen Data

\begin{tabular}{|c|c|c|c|}
\hline $\begin{array}{l}\text { Mineral and identi- } \\
\text { fying number }\end{array}$ & Location & $\begin{array}{l}\text { Ref, no, of } \\
\text { X-ray patterns }\end{array}$ & Quantity \\
\hline $\begin{array}{l}\text { Xenotime } \\
\text { USNM } 105008\end{array}$ & Luwangashi & --- & $\begin{array}{l}\text { USGS-XS } \\
\text { USMM-25 }\end{array}$ \\
\hline Xenotime & $\begin{array}{l}\text { Washington Heights, } \\
\text { New York, N.Y. }\end{array}$ & Inv. 2708 & $-\infty$ \\
\hline $\begin{array}{l}\text { Xenotime } \\
\text { HMM }\end{array}$ & $\begin{array}{l}\text { Washington Heights, } \\
\text { New York, N.Y. }\end{array}$ & Har. 5838 & -- \\
\hline $\begin{array}{l}\text { Xenotime } 2 / \\
\text { USNM } 82391\end{array}$ & $\begin{array}{l}\text { Pikes Peak, } \\
\text { EI Paso County, Colo. }\end{array}$ & $-\cdots$ & $-\infty$ \\
\hline
\end{tabular}

If Hutton, C. O., Am. Mineralogist 32, 141 (1947). Kraus, M, and Reitinger, Zeitschr. Kristallographie, 34, 268 (1901)

2) Penfield, S. L., Am. Jour. Sci., 3rd ser., 45, 398-399 (1893).

YTTRIALITE - Silicate of Th and Y metals chiefly

Specimen Data

\begin{tabular}{|c|c|c|c|}
\hline $\begin{array}{l}\text { Mineral and identi- } \\
\text { fying number }\end{array}$ & Location & $\begin{array}{l}\text { Ref. no. of } \\
\text { X-ray patterns }\end{array}$ & Quantity \\
\hline $\begin{array}{l}\text { Yttrialite } \\
\text { USNM C3094 }\end{array}$ & $\begin{array}{l}\text { Llano County, } \\
\text { Texas }\end{array}$ & $\begin{array}{l}\text { TE } 738 \\
\text { Ignited }\end{array}$ & $\begin{array}{l}\text { USGS-XS } \\
\text { USNM-2O g }\end{array}$ \\
\hline $\begin{array}{l}\text { Yttwialite } \\
\text { USNM R5878 }\end{array}$ & $\begin{array}{l}\text { Llano County, } \\
\text { Texas }\end{array}$ & $\ldots$ & $\begin{array}{l}\text { USGS-XS } \\
\text { USNM-20 g }\end{array}$ \\
\hline $\begin{array}{l}\text { Yttrialite I/ } \\
\text { USNM } 85070 ?\end{array}$ & $\begin{array}{l}\text { Barringer Hill,; } \\
\text { Llano County, Texas }\end{array}$ & -- & $\mathrm{USNM}_{-30} \mathrm{~g}$ \\
\hline
\end{tabular}

If Hillebrand, W. F., Am. Jour. Sci. 4th ser., 13, 145 (1902). 


$$
\begin{gathered}
\text { YTTROCRASTTE }(\mathrm{Y}, \mathrm{Th}, \mathrm{U}, \mathrm{Ca})_{2}(\mathrm{TI})_{4} \mathrm{O}_{11} \text { ? } \\
\text { Optics }
\end{gathered}
$$

In transmitted light, rich amber to light yellow. In part metamict and isotropic; in part weakly anisotropic. $n$ ranges from 2.12 to $2.15 .1 /$

$$
\text { Specimen Data }
$$

$$
\begin{aligned}
& \text { Mineral and identi- Location Ref. no. of Quantity } \\
& \text { fying number } \\
& \text { X-ray patterns } \\
& \text { YTTROTANTALITE }(\mathrm{Fe}, \mathrm{Y}, \mathrm{U})(\mathrm{Cb}, \mathrm{Ta}) \mathrm{O}_{4} \\
& \text { Optics }
\end{aligned}
$$

Transmits light in thin splinters. Isotropic (metamict), becomes crystalline on heating. In section red brown and isotroric in $=2.15 \pm$ 0.02 .1

$$
\text { Specimen Data }
$$
Mineral and identi- Location fying number
Ref. no. of
X-ray patterns
Ytrotantalite
USNM R7ILI
Itterby,
$\ldots$
Sweden
Quantity
Yttrotantalite
Ytterby,
HMM 87851
Sweden
Har. 1887
Har. $190^{\circ}$
Ignited
USGS-XS
USNM-150 g
1 Larsen, E. S., U. S. Geol. Survey Bu.17. 679, I58 (1921)。

\section{ZIRCON $\mathrm{ZrSiO}_{4}$}

Specimen Data

$\begin{array}{llll}\begin{array}{l}\text { Mineral and identi- } \\ \text { fying number }\end{array} & \text { Location } & \begin{array}{c}\text { Ref. no. of } \\ \text { X-ray patterns }\end{array} & \text { Quantity } \\ \begin{array}{l}\text { Zircon } \\ \text { HMM } 91500\end{array} & \begin{array}{l}\text { Renfrew } \\ \text { Couity, Ontario }\end{array} & \text { Har. } 7979\end{array}$




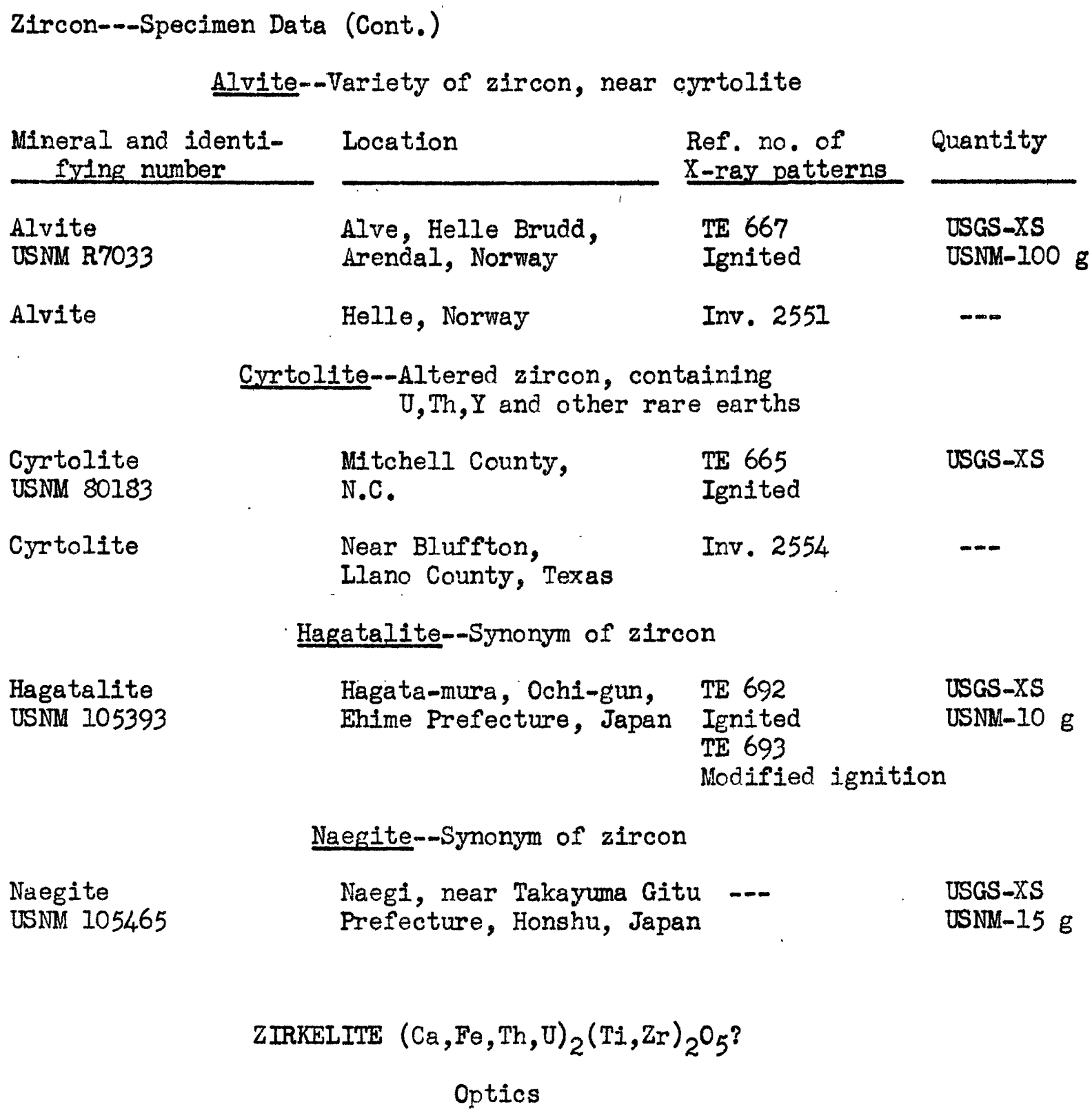

Cyrtolite

USNM 80183

Cyrtolite

Hagatalite

USNM 105393

Naegite

USNM 105465

Mitchell County, N.C.

Near Bluffton, Llano County, Texas

Hagatalite--Synonym of zircon

Hagata-mura, Ochi-gun, TE 692

Ehime Prefecture, Japan Ignited TE 693

Modified ignition

Naegite--Synonym of zircon

Naegi, near Takayuma Gitu Prefecture, Honshu, Japan

USGS-XS

USNM-15 $g$

$$
\begin{gathered}
\text { ZIRKELITE }(\mathrm{Ca}, \mathrm{Fe}, \mathrm{Th}, \mathrm{U})_{2}(\mathrm{Ti}, \mathrm{Zr})_{2} \mathrm{O}_{5} \text { ? } \\
\text { Optics }
\end{gathered}
$$

Transparent in very thin splinters, with a dark-brown or reddishbrown color. $n=2.19 . \pm 0.01 .1$

\begin{tabular}{|c|c|c|c|}
\hline $\begin{array}{l}\text { Mineral and identi- } \\
\text { fying number }\end{array}$ & Location & $\begin{array}{l}\text { Ref. no. of } \\
\text { X-ray patterns }\end{array}$ & Quantity \\
\hline $\begin{array}{l}\text { Zirkelite } \\
\text { USNM R5026 }\end{array}$ & $\begin{array}{l}\text { Jacuipiranga, } \\
\text { Săo Paulo, Brazil }\end{array}$ & --- & $\begin{array}{l}\text { USGS-X } \\
\text { USNM-1 }\end{array}$ \\
\hline
\end{tabular}

$$
\text { Specimen Data }
$$

If Larsen, E. S., U. S. Geol. Survey Bull. 679, 160 (1921). 
GROUP C. SYNTHETIC URANIUM COMPOUNDS PREPARED BY THE U. S. GEOLOGICAL SURVE URANYL ARSENATES

Compound

Ca uranyl arsenate Preparation 20

Cu uranyl arsenate
Method of synthesis

Precipitation from solution

Precipitation from solution
$\mathrm{Na}, \mathrm{Ca}$ uranyl carbonate Precipitation from solution

Ca, Mg uranyl carbonate Precipitation from solution

Mg uranyl carbonate
Ref. no. of $X-$ ray patterns

TE 97
Inv. 2869

Inv. 4266

Inv. 2868

URANYL PHOSPHATES I/

Ca uranyl phosphate Preparation 13

Na uranyl phosphate

Ba uranyl phosphate

Mn uranyl phosphate

Cu uranyl phosphate

Ni uranyl phosphate

$\mathrm{Pb}$ uranyl phosphate

Mg uranyl phosphate
Precipitation from solution

TE 58
Base exchange
from $\mathrm{Ca}$ compound

Base exchange from lia compound

Base exchange from $\mathrm{Na}$ compound

Base exchange from $\mathrm{Na}$ compound

Base exchange from $\mathrm{Na}$ compound

Base exchange from $\mathrm{Na}$ compound

Base ex change from $\mathrm{Na}$ compound 


\section{URANYL VANADATES $2 /$}

Compound

$\mathrm{Na}$ uranyl vanadate

Preparation 16

$\mathrm{K}$ uranyl vanadate

Preparation 15

Ca uranyl vanadate

Preparation 6

$\left(\mathrm{NH}_{4}\right)$ uranyl vanadate Preparation 9

$\mathrm{Cu}$ uranyl vanadate

Preparation 18

Zn uranyl vanadate Preparation 34

Sr uranyl vanadate

Preparation 12

Ba uranyl vanadate

Preparation 8

TI uranyl vanadate

Preparation 35
Method of synthesis

Precipitation from solution

Precipitation from solution

Precipitation from solution

Precipitation from solution

Base exchange from $\mathrm{Ca}$ compound

Base exchange

from $\mathrm{Ca}$ compound

Precipitation from solution

Precipitation from solution

Base exchange from $\mathrm{Ca}$ compound
Ref. no. of

$X$-ray patterns

TE 68

- TR 57

TE 19

TE 22

TE 67

TE 227

TE 37

TE 21

TE 229

IJ Fairchild, J. G., Am. Mineralogist 1/4, 265-275 (1929).

2) Murata, K. J., et al., U. S. Geol. Survey TEI Rept. 107 (1950). 